\title{
LIANAS OF MEXICO
}

\author{
Guillermo Ibarra-Manríquez ${ }^{1,3}$, Francisco Javier Rendón-Sandoval' ${ }^{1}$, \\ Guadalupe Cornejo-Tenorio and Pablo Carrillo-Reyes² \\ ${ }^{1}$ Instituto de Investigaciones en Ecosistemas y Sustentabilidad, Universidad Nacional Autónoma de México. \\ Morelia, Michoacán, México. \\ ${ }^{2}$ Instituto de Botánica, Departamento de Botánica y Zoología, Centro Universitario de Ciencias Biológicas y \\ Agropecuarias, Universidad de Guadalajara. Zapopan, Jalisco, México. \\ ${ }^{3}$ Corresponding author: gibarra@cieco.unam.mx
}

\begin{abstract}
Through an exhaustive literature review, field exploration and revision of herbaria, the presence of 861 native species of lianas in Mexico is documented, pertaining to 250 genera and 59 families. A total of $25.9 \%(n=224)$ of the species and three genera are endemic to Mexico. The 15 most diverse families account for $80.1 \%$ of the species and $85.7 \%$ of endemics. The 18 richest genera comprise $38.1 \%(n=328)$ of the species and approximately $50 \%$ of the endemic. Chiapas is the state with the greatest diversity because it includes $65.4 \%$ of the total species, whereas Oaxaca is the richest state in terms of endemism (49.6\%). Regardless of their area, the most diverse states are also notable for their endemism. The lianas found in only one state encompassed 16.5 and $26 \%$ of the total number of species and endemics, respectively. Among the five recognized biomes in Mexico, the tropical humid forest and the tropical seasonally dry forest stand out for their number of species $(n=692,80.3 \%)$ and number of endemics $(n=134,59.8 \%)$, respectively. At the level of vegetation type, the tropical deciduous forest ranks first in both attributes. The number of lianas species decreased significantly with altitude. Our results demonstrate the importance of the contribution of lianas to the richness and endemism of Mexican flora and justify the development of initiatives to ensure their long-term conservation.
\end{abstract} Key words: altitudinal gradients, biomes, diversity, endemism, vegetation types.

Resumen: Por medio de una exhaustiva revisión bibliográfica, trabajo de campo y la revisión de ejemplares de herbario, se documenta la presencia de 861 especies de lianas nativas, incluidas en 250 géneros y 59 familias. El $25.9 \%(n=224)$ de las especies y tres géneros son endémicos de México. Las 15 familias más diversas concentran el $80.1 \%$ del total de especies y el $85.7 \%$ de las endémicas. Los 18 géneros con mayor riqueza concentran $38.1 \%(n=328)$ de las especies de lianas y cerca del $50 \%$ de las endémicas. A nivel de estados, Chiapas es el más sobresaliente, ya que registró el $65.4 \%$ del total de especies, mientras que Oaxaca lo fue para las endémicas (49.6\%). Los estados muestran una alta congruencia entre la riqueza de especies y grado de endemismo, atributos que no se explican por el área que éstos presentan. Las lianas restringidas a un solo estado representaron el 16.5 y $26 \%$ del número total de especies y endémicas, respectivamente. Entre los cinco biomas reconocidos para México, el bosque tropical húmedo destacó por su número de especies $(n=692,80.3 \%)$, mientras que el bosque tropical estacionalmente seco hace lo propio para las endémicas $(n=134,59.8 \%)$. Por tipo de vegetación, la primera posición para ambos atributos es para el bosque tropical caducifolio. El número de especies de lianas decreció significativamente con la altitud. Los resultados del presente estudio demuestran que la contribución de las lianas a la riqueza y endemismo de la flora de México es relevante, lo cual justifica iniciativas particulares que permitan asegurar su conservación a largo plazo.

Palabras clave: biomas, diversidad, endemismo, gradientes de altitud, tipos de vegetación.

$T^{n}$ he climbing habit has evolved on many occasions in plants and has been detected in at least 133 families of Angiosperms (Gentry, 1991a; Schnitzer and Bongers, 2002; Gallagher and Leishman, 2012). The independent evolution of this habit in so many lineages could be explained because climber species invest fewer resources in the development of their stems compared to erect plants, enabling this group to direct these resources toward other important functions, such as growth or water capture (Schnitzer et al., 2005; Wyka et al., 2013). Lianas play an important role in the taxonomic and functional diversity in tropical forests, especially during early succession stages, as well as interaction with a high number of pollinators and frugivores (Gentry 1982, 1991a, b; Solórzano et al., 2002; Schnitzer and Bongers, 
2002; Letcher and Chazdon, 2012). For example, the complete floristic inventories of particular locations demonstrate that this growth form can account for between 8 and $24 \%$ of the woody species (Gentry and Dodson, 1987; Ibarra-Manríquez and Sinaca, 1995; Meave et al., 2008). Inventories in plots of 0.1 ha have revealed that an average of $18 \%$ of stems with DBH (diameter at breast height) $\geq 2.5 \mathrm{~cm}$ belong to lianas (Gentry, 1991a) and in similar censuses in Mexican tropical dry forests are absent or may represent up to $25 \%$ of the species present, with an average of $10.4 \pm$ S.D. 7.1 (Lott et al., 1987; Trejo and Dirzo, 2002; Pineda-García et al., 2007; Martínez-Cruz et al., 2013).

Due mainly to the magnitude of Mexican floristic diversity, which is estimated to be at least 22,000 species of Angiosperms (Rzedowski, 1991a; Espejo-Serna et al., 2004a; Villaseñor and Ortiz, 2014), the species richness of all of the present growth forms is not equally known. Undoubtedly, the trees and the epiphytes are those that are best characterized because these are the subjects of relatively recent floristic publications (e.g. Ibarra-Manríquez et al., 1995; Espejo-Serna et al., 2004b; Hágsater et al., 2005; Cué-Bär et al., 2006a; Padilla-Velarde et al., 2006; Soto-Arenas et al., 2007; González-Espinosa et al., 2011; Ricker et al., 2013). However, no such compendia exist for the lianas.

An important source of information to know the richness of lianas are the regional floristic projects [e.g. Flora del Bajío y de Regiones Adyacentes (Rzedowski and Calderón, 1993; Calderón et al., 2004), Flora de Veracruz (PérezCueto, 1995; Ortega and Ortega, 1997) or Flora del Valle de Tehuacán-Cuicatlán (Juárez-Jaimes and Lozada, 2003; Alvarado-Cárdenas, 2004)], local floristic inventories (e.g. Ibarra-Manríquez and Sinaca, 1995, 1996a, b; Lott, 2002; Meave et al., 2008) and checklist available to Mexico for some families with many lianas representatives (Borhidi, 2006; Juárez-Jaimes et al., 2007; Anderson, 2013).

Specimens that are deposited within herbaria also are another key tool to know diversity of Mexican lianas, although a critical taxonomic review of the material is required. Nonetheless, this task is generally difficult to implement for several reasons: (i) lianas are underrepresented in herbaria because of collection difficulty, (ii) specimens often lack structures that are critical for accurate identification, (iii) collections, in many cases, are not revised by specialists, and (iv) there is a scarcity or absence of monographs of several climber families or genera.

On the other hand, estimates of the taxonomic richness of Mexican lianas differ because Rzedowski (1991a) suggested that there are 60 genera and 300 species, while Ibarra-Manríquez et al. (2015) indicated 214 and 754 taxa in these two taxonomic categories, respectively. The difference between these figures is due, among other factors, to the concept of the liana that is held by each of these authors, as well as the sparse floristic knowledge that exists regarding this growth form, which difficult its inventory. To accurately estimate the contribution of lianas to floristic diversity in Mexico, our fundamental objective is to list the species of lianas in this country, indicating the states, vegetation types, and altitudinal intervals in which they are found, as well as the species that restrict their geographic distribution to Mexico (endemic species).

\section{Study area}

Mexico has a land area of close to two million $\mathrm{km}^{2}$. The country has been subject to complex geological changes throughout its history (Ferrusquía-Villafranca, 1993) that have caused the topography, with the exception of the Yucatán peninsula, to be dominated by mountains. Rzedowski (1978) states that only $35 \%$ of the country is below $500 \mathrm{~m}$ asl, while more than half is above $1,000 \mathrm{~m}$ altitude, with the highest peaks found along the length of the Trans-Mexican Volcanic Belt, where the volcano Pico de Orizaba (Citlaltépetl) stands at 5,636 m elevation. Such a heterogeneous landscape, along with the position of the country astride the Tropic of Cancer, have produced a broad range of climates and vegetation types, including practically all of those that are recognized worldwide (Rzedowski 1978, 1991a). According to the Köppen classification, the climates that affect the greatest territorial area are (Kottek et al., 2006): A (tropical wet, with the mean temperature in the coldest month greater than $18^{\circ} \mathrm{C}$ ), B (dry, with a mean temperature in the coldest month greater or less than $18^{\circ} \mathrm{C}$ and a mean annual temperature between 18 and $22^{\circ} \mathrm{C}$ ), $\mathrm{C}$ (temperate wet, with a mean temperature in the coldest month between -3 and $18{ }^{\circ} \mathrm{C}$ and in the hottest month greater than $10^{\circ} \mathrm{C}$ ) and, in very reduced areas and high altitude locations of the volcanoes of central Mexico, E (cold, with a mean temperature in the hottest month less than $10^{\circ} \mathrm{C}$ ). Rzedowski (1978) recognized four biogeographic regions (Pacific North American, Montane Mesoamerican, Xerophytic Mexican and Caribbean) that include 17 provinces. The number of recognized vegetation types range from ten (Rzedowski, 1978) to 32 (Miranda and Hernández-X., 1963). These publications describe the physiognomic characteristics and principal floristic components of these plant communities, as well as the climates and soil types in which they are found. In global terms, floristic affinities are more meridional than boreal, and, according to the fossil evidence, their origin goes back to at least the Cenozoic era, some 65 million years ago (Rzedowski, 1991a, b).

\section{Materials and methods}

There is no accurate definition by which to categorize liana species; however, they are generally described as climbing plants with woody stems that cannot remain upright autonomously once they have germinated in the soil (Gentry, 1991a; Bongers et al., 2002). Unfortunately, these basic at- 
tributes are not known for many species. For example, the designation of a plant as woody is not simple because this characteristic may be presented in individuals with very thin stems (i.e. $\leq 1 \mathrm{~mm}$ diameter) or only toward the base of stems. This information is rarely included in floristic publications that report climber species or in their descriptions of herbarium specimens and is even often neglected in taxonomic descriptions. The need of information is even more acute for germination in the soil, which is the main characteristic by which to separate lianas from woody climbing hemiepiphytes because the latter only germinate on the trunks or branches of trees (Gentry, 1991a). Another aspect that is difficult to evaluate is whether the plants are erect or require support for growth because this can vary with the age of the plants or in different vegetation types. An example of this situation is Salacia cordata (Miers) Mennega (Celastraceae), which is a liana that we can found in the upper canopy in the tropical evergreen forest but when young, is as small erect tree that grows up to a height of $3 \mathrm{~m}$ in the Los Tuxtlas Tropical Biological Station in Veracruz. At this same site grows the liana Celtis iguanaea (Jacq.) Sarg. (Cannabaceae), which in the tropical deciduous forest of the Chamela-Cuixmala region in Jalisco, can exhibit the liana habit or may grow as a shrub.

To assign a native species as liana (naturalized species were excluded), we considered: (1) the floristic-taxonomic literature (Appendix 1) and specimens that were deposited in herbaria (IEB, MEXU, MO, and XAL) where the species is described as a liana or woody climber, even if this condition is only presented towards the stem base, and (2) field observations in different parts of the country examining climbing species to evaluate the degree of woodiness (Figure 1) and to collect specimens with flowers and/or fruits in order to identify correctly the species (Figure 2 and 3). Consequently, the list also includes: (i) species that exhibit a growth habit that is similar to that of the lianas but do not germinate in the soil (hemiepiphytes), (ii) those that have a highly lignified atactostele (monocots, members of the families Arecaceae and Smilacaceae) or (iii) scandent shrubs with climbing branches that are greater than $5 \mathrm{~m}$ in length. Therefore, one or more of the following three growth forms were assigned to the species (Appendix 2): liana (including monocots), hemiepiphytes, and scandent shrubs. The identification of the specimens in the herbaria was verified by specialists or by the authors using monographs.

The altitude, vegetation type, and geographical distribution were obtained from the examined literature and herbarium specimens. Endemic species were those lianas only found in Mexico, whereas microendemics were identified such those species that are recorded in only one state of the country. Vegetation types and biomes were assigned according to the classifications of Rzedowski (1978) and Villaseñor and Ortiz (2014), respectively. In states with greater taxonomic richness, an analysis was conducted to determine whether the size of their territory could be a factor that explains this attribute. For this analysis, a weighted ratio was obtained between the number of families, genera, and species (total, endemics and microendemics) and the $\log _{10}$ of the area of each state. This value approaches the average richness of a given taxon in an area of $10 \mathrm{~km}^{2}$ (Rejmánek, 1996). The authors of the species and the publication in which they were described were recorded from the consulted literature (Appendix 1) and/or the database Tropicos (Tropicos, 2014).

\section{Results}

Taxonomic richness. Mexican native lianas are included in 59 families, 250 genera, and 861 species (Appendix 2). Among the three growth form categories that were recognized in this study, the lianas were the most important $(n=$ 789 species), followed by the scandent shrubs $(n=246)$ and the hemiepiphytes $(n=26)$. A total of 15 families contributed differentially to the taxonomic richness of lianas because account for $70.4 \%$ of the genera, $80.1 \%$ of the total number of species, $85.7 \%$ of the endemics and $84.2 \%$ of the microendemics (Table 1). Apocynaceae dominated these four groups, followed by Fabaceae, except for the microendemic species, where second place was taken by Asteraceae. In contrast, 15 families were each represented by one species (Appendix 2).

Table 1. Number of genera and species for the most diverse families of lianas in Mexico.

\begin{tabular}{lllll}
\hline \multirow{2}{*}{ Family } & Genera (\%) & Total & Endemics & $\begin{array}{l}\text { Micro- } \\
\text { endemics }\end{array}$ \\
\hline Apocynaceae & $32(12.8)$ & $158(18.3)$ & $71(31.7)$ & $17(29.8)$ \\
Fabaceae & $26(10.4)$ & $94(10.9)$ & $24(10.7)$ & $4(7.0)$ \\
Convolvulaceae & $12(4.8)$ & $67(7.8)$ & $18(8.0)$ & $4(7.0)$ \\
Asteraceae & $23(9.2)$ & $61(7.1)$ & $19(8.5)$ & $8(14.0)$ \\
Malpighiaceae & $14(5.6)$ & $57(6.6)$ & $17(7.6)$ & $6(10.5)$ \\
Sapindaceae & $6(2.4)$ & $48(5.6)$ & $9(4.0)$ & $7(12.3)$ \\
Bignoniaceae & $17(6.8)$ & $41(4.8)$ & $1(0.4)$ & $0(0.0)$ \\
Solanaceae & $6(2.4)$ & $35(4.1)$ & $9(4.0)$ & $1(1.8)$ \\
Passifloraceae & $1(0.4)$ & $27(3.1)$ & $3(1.3)$ & $0(0.0)$ \\
Vitaceae & $5(2.0)$ & $26(3.0)$ & $2(0.9)$ & $1(1.8)$ \\
Rubiaceae & $10(4.0)$ & $21(2.4)$ & $5(2.2)$ & $1(1.8)$ \\
Aristolochiaceae & $1(0.4)$ & $18(2.1)$ & $7(3.1)$ & $5(8.8)$ \\
Menispermaceae & $6(2.4)$ & $13(1.5)$ & $1(0.4)$ & $0(0.0)$ \\
Celastraceae & $8(3.2)$ & $12(1.4)$ & $1(0.4)$ & $0(0.0)$ \\
Cucurbitaceae & $9(3.6)$ & $12(1.4)$ & $5(2.2)$ & $0(0.0)$ \\
Subtotal & $176(70.4)$ & $690(80.1)$ & $192(85.7)$ & $48(84.2)$ \\
Other 43 families & $74(29.6)$ & $171(19.9)$ & $32(14.3)$ & $9(15.8)$ \\
\hline & & & &
\end{tabular}



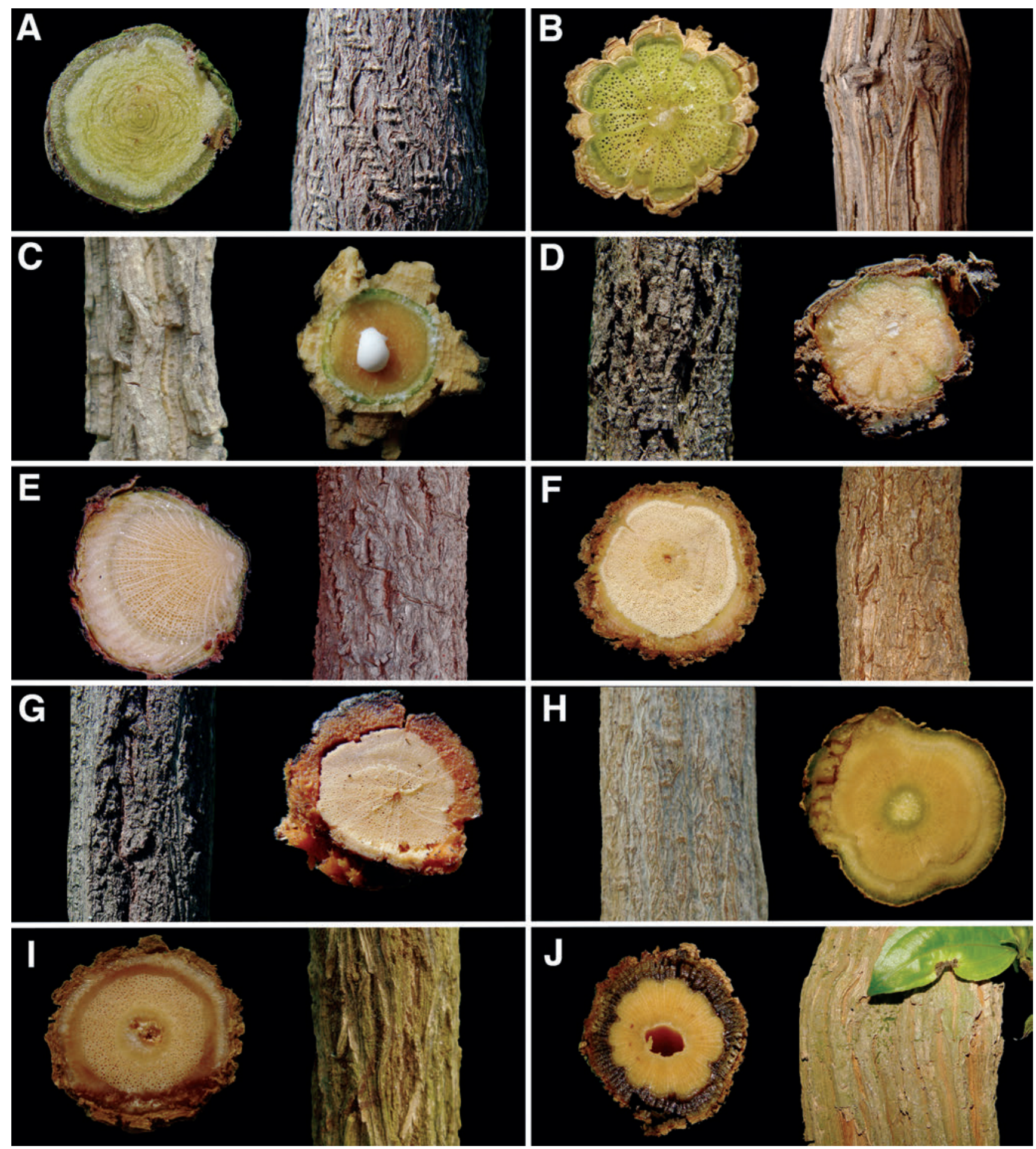

Figure 1. Liana stems and their cross-section in species registered in the present study. A) Canavalia villosa, B) Clematis dioica, C) Matelea crenata, D) Nissolia microptera, E) Parthenocissus quinquefolia, F) Passiflora subpeltata, G) Serjania racemosa, H) Solanum dulcamaroides, I) Trichostigma octandrum, and J) Tuxtla pittieri.

The 18 genera with the greatest richness account for $38.1 \%$ of the species (Table 2), with slightly higher percentages of endemic and microendemic taxa $(45.1 \%$ and $50.9 \%$, respectively). The reduced dominance of the most speciose genera compared to that documented for the families occurs because approximately half of genera present only one species $(n=114)$. The genus with the greatest number of species ( $n=43)$ is Ipomoea (Convolvulaceae), whereas Ser- 

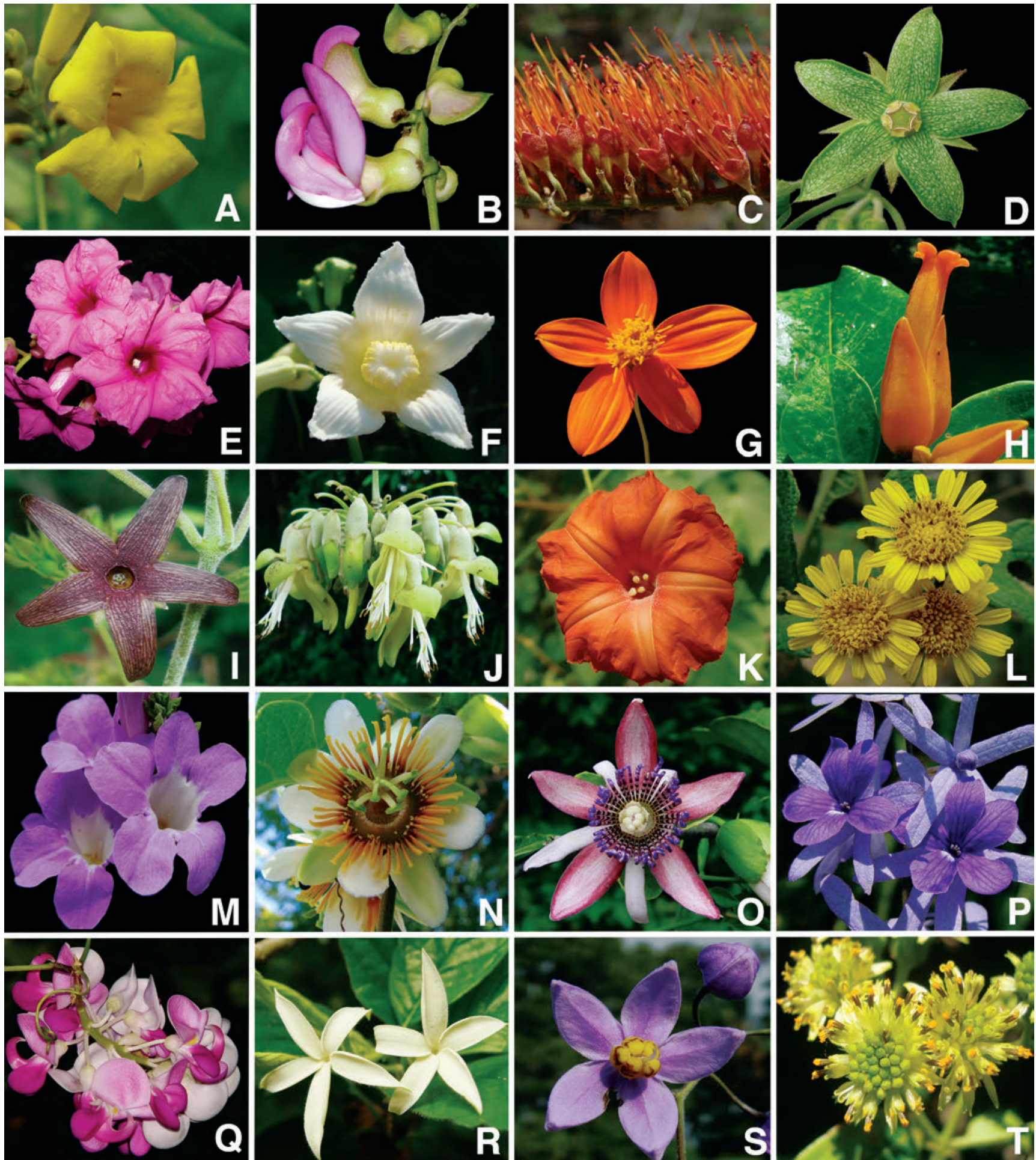

Figure 2. Flowers of liana species included in the present study. A) Adenocalymma inundatum, B) Canavalia villosa, C) Combretum farinosum, D) Gonolobus grandiflorus, E) Ipomoea phillomega, F) Hanburia mexicana, G) Hidalgoa ternata, H) Juanulloa mexicana, I) Matelea crenata, J) Mucuna argyrophylla, K) Operculina pteripes, L) Otopappus epaleaceus, M) Paragonia pyramidata, N) Passiflora holosericea, O) P. ambigua, P) Petrea volubilis, Q) Ramirezella strobilophora, R) Randia retroflexa, S) Solanum dulcamaroides, and T) 
Guillermo IbarRa-ManRíQueZ ET AL.

Table 2. Number of species and family for the most diverse genera of lianas in Mexico.

\begin{tabular}{|c|c|c|c|c|}
\hline \multirow[b]{2}{*}{ Genera } & \multirow[b]{2}{*}{ Family } & \multicolumn{3}{|c|}{ Species (\%) } \\
\hline & & Total & Endemics & Microendemics \\
\hline Ipomoea & Convolvulaceae & $43(5.0)$ & $12(5.4)$ & $2(3.5)$ \\
\hline Serjania & Sapindaceae & $33(3.8)$ & $8(3.6)$ & $6(10.5)$ \\
\hline Passiflora & Passifloraceae & $27(3.1)$ & $3(1.3)$ & $0(0.0)$ \\
\hline Marsdenia & Apocynaceae & $25(3.0)$ & $13(5.8)$ & $3(5.3)$ \\
\hline Matelea & Apocynaceae & $23(2.7)$ & $11(5.0)$ & $2(3.5)$ \\
\hline Solanum & Solanaceae & $20(2.3)$ & $6(2.7)$ & $1(1.8)$ \\
\hline Aristolochia & Aristolochiaceae & $18(2.1)$ & $7(3.0)$ & $5(8.8)$ \\
\hline Gonolobus & Apocynaceae & $18(2.1)$ & $6(2.7)$ & $1(1.8)$ \\
\hline Metastelma & Apocynaceae & $18(2.1)$ & $10(4.5)$ & $2(3.5)$ \\
\hline Cissus & Vitaceae & $13(1.5)$ & $1(0.5)$ & $0(0.0)$ \\
\hline Dictyanthus & Apocynaceae & $13(1.5)$ & $10(4.5)$ & $3(5.3)$ \\
\hline Mandevilla & Apocynaceae & $12(1.4)$ & $5(2.2)$ & $1(1.8)$ \\
\hline Otopappus & Asteraceae & $12(1.4)$ & $7(3.1)$ & $2(3.5)$ \\
\hline Clematis & Ranunculaceae & $11(1.3)$ & $2(0.9)$ & $0(0.0)$ \\
\hline Smilax & Smilacaceae & $11(1.3)$ & $0(0.0)$ & $0(0.0)$ \\
\hline Fridericia & Bignoniaceae & $11(1.3)$ & $0(0.0)$ & $0(0.0)$ \\
\hline Mikania & Asteraceae & $10(1.2)$ & $1(0.4)$ & $1(1.8)$ \\
\hline Paullinia & Sapindaceae & $10(1.2)$ & $0(0.0)$ & $0(0.0)$ \\
\hline Subtotal & & $328(38.1)$ & $101(45.1)$ & $29(50.9)$ \\
\hline Other 231 genera & & $533(61.9)$ & $123(54.9)$ & $28(49.1)$ \\
\hline
\end{tabular}

jania (Sapindaceae) and Passiflora (Passifloraceae) have 33 and 27 species (Table 2); the first place in the endemic species is Marsdenia (Apocynaceae), followed by Ipomoea and Matelea (Apocynaceae). Finally, standing out in terms of microendemic species are Serjania and Aristolochia (Aristolochiaceae), with six and five species, respectively.

Geographic distribution. A total of $25.9 \%(n=224)$ of the recorded species of lianas are endemic to Mexico, while only three genera $(1.2 \%)$ are included in this category (Balsas J.Jiménez-Ram. \& K.Vega, Sapindaceae; Peponopsis Naudin, Cucurbitaceae; and Thoreauea J.K. Williams, Apocynaceae). Of whole sample of Mexican lianas, 57 species were recorded as microendemic (Table 3, Appendix 2 ), including those that were assigned to two endemic genera (Balsas guerrerensis Cruz-Durán \& K.Vega restricted to Guerrero, Thoreaua aberrans J.F. Morales to Veracruz, T. guerrerensis Diego \& Lozada-Pérez to Guerrero and T. paneroi J.K. Williams to Oaxaca).

Chiapas is highlighted at the state level (Table 3 ) because almost all of the families are recorded within its area $(n=$ $57,97 \%)$, as well as $85.2 \%(n=213)$ of the genera, and $65.2 \%(n=562)$ of species. In these categories, Oaxaca presents very similar figures and therefore normally follows Chiapas in hierarchical order. In general, Veracruz occupies third place for these attributes of taxonomic richness, with the exception of the microendemic species, where second place is shared with Chiapas. Oaxaca encompass the greatest number of endemic species, with close to half of the total found in Mexico ( $n=111)$, followed by Jalisco, Guerrero, and Michoacán, the percentages of which range from 34.8 to $39.7 \%$. Unexpectedly, the differences in area among the seven states that were cited in this section did not affect the position they occupy in these comparisons of diversity because the ratio values between the richness of taxa and the $\log _{10}$ of the area of each state follow the same pattern as described above using the number of species (Table 3 ).

The frequency with which the lianas have been recorded in the 32 states of Mexico indicate that $142(16.5 \%)$ and $109(12.6 \%)$ species are found in only one and two states, respectively (Table 4). Of the total species, $60.3 \%$ have been found in up to five states, and only 18 (2\%) exist in more than 20 states. The most widely distributed species are Cardiospermum halicacabum L. (Sapindaceae, $n=31$ states), Toxicodendron radicans (L.) Kuntze (Anacardiaceae, $n=27$ ), and Canavalia villosa Benth. (Fabaceae, $n=$ 26). Restriction at state level is more noteworthy in the 224 endemic species because $40.6 \%$ are found in one or two states, and $29 \%$ ( $n=65$ species) do not occupy more than five states (Table 4). The endemic lianas with the greatest geographic distribution are Lonicera pilosa (Kunth) Willd. ex Kunth (Caprifoliaceae; $n=21$ states), followed by Funastrum pannosum Schltr. (Apocynaceae), and Nissolia microptera Poir. (Fabaceae), both found in 19 states.

Biomes, vegetation types and altitude. The richness of lianas species is dissimilar among the five recognized biomes of Mexico (Table 5) because the tropical humid forest clearly is 

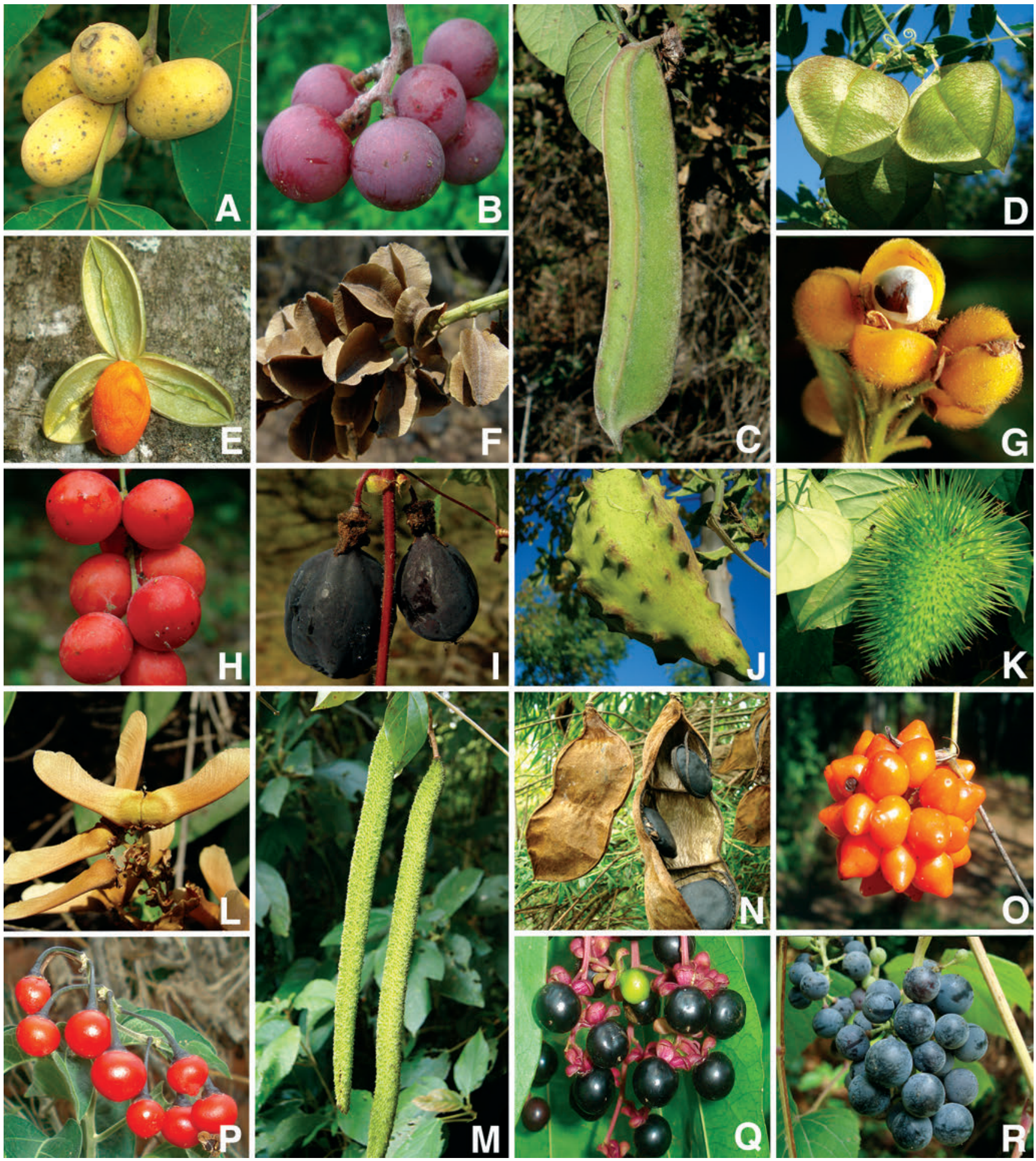

Figure 3. Fruits of liana species reported to Mexico. A) Abuta panamensis, B) Ampelocissus acapulcensis, C) Canavalia villosa, D) Cardiospermum halicacabum, E) Celastrus pringlei, F) Combretum farinosum, G) Davilla kunthii, H) Disciphania calocarpa, I) Passiflora exsudans, J) Gonolobus chloranthus, K) Hanburia mexicana, L) Heteropterys laurifolia, M) Mansoa verrucifera, N) Mucuna argyrophylla, O) Smilax pringlei, P) Solanum dulcamaroides, Q) Trichostigma octandrum, and R) Vitis tiliifolia. 
Table 3. Families, genera and species of lianas per state in Mexico. Taxonomically more diverse states are highlighted in bold and the ratio between the number of each taxon and the log base10 of the area of each state is shown in parentheses. Surface of states highlighted in bold is: Chiapas, 73,311 km²; Guerrero, 63,596 km²; Jalisco, 78,588 km²; Michoacán, 58,599 km²; Oaxaca, 93,757 km²; Tabasco, 24,731km²; Veracruz, $71,826 \mathrm{~km}^{2}$.

\begin{tabular}{|c|c|c|c|c|c|}
\hline \multirow[t]{2}{*}{ States } & \multirow[t]{2}{*}{ Families } & \multirow[t]{2}{*}{ Genera } & \multicolumn{3}{|c|}{ Species } \\
\hline & & & Total & Endemics & Microendemics \\
\hline Aguascalientes & 9 & 13 & 14 & 6 & 0 \\
\hline Baja California & 10 & 11 & 13 & 3 & 0 \\
\hline Baja California Sur & 16 & 24 & 34 & 15 & 2 \\
\hline Campeche & 36 & 115 & 216 & 8 & 0 \\
\hline Chiapas & $57(11.7)$ & $213(43.8)$ & $562(115.5)$ & $38(7.8)$ & $10(2.1)$ \\
\hline Chihuahua & 18 & 32 & 42 & 12 & 0 \\
\hline Coahuila & 12 & 23 & 31 & 9 & 0 \\
\hline Colima & 36 & 97 & 158 & 43 & 1 \\
\hline Distrito Federal & 15 & 24 & 34 & 15 & 0 \\
\hline Durango & 21 & 34 & 48 & 15 & 0 \\
\hline Guanajuato & 19 & 38 & 53 & 22 & 0 \\
\hline Guerrero & $41(8.5)$ & $133(27.7)$ & 305 (63.5) & $88(18.3)$ & $5(1.0)$ \\
\hline Hidalgo & 35 & 78 & 134 & 31 & 0 \\
\hline Jalisco & $41(8.4)$ & $133(27.2)$ & 292 (59.6) & $89(18.2)$ & $6(1.2)$ \\
\hline México & 31 & 88 & 157 & 48 & 1 \\
\hline Michoacán & $41(8.6)$ & $115(24.1)$ & 255 (53.5) & $78(16.4)$ & $2(0.4)$ \\
\hline Morelos & 32 & 81 & 137 & 33 & 0 \\
\hline Nayarit & 35 & 97 & 162 & 39 & 2 \\
\hline Nuevo León & 19 & 40 & 56 & 15 & 0 \\
\hline Oaxaca & $56(11.3)$ & $210(42.2)$ & $541(108.8)$ & $111(22.3)$ & $12(2.4)$ \\
\hline Puebla & 34 & 92 & 179 & 57 & 2 \\
\hline Querétaro & 33 & 77 & 134 & 5 & 0 \\
\hline Quintana Roo & 34 & 106 & 198 & 32 & 0 \\
\hline San Luis Potosí & 32 & 79 & 133 & 48 & 1 \\
\hline Sinaloa & 33 & 86 & 149 & 28 & 0 \\
\hline Sonora & 25 & 47 & 70 & 31 & 1 \\
\hline Tabasco & $41(9.3)$ & $133(30.3)$ & $265(60.3)$ & $4(0.9)$ & $1(0.2)$ \\
\hline Tamaulipas & 33 & 76 & 130 & 18 & 1 \\
\hline Tlaxcala & 7 & 8 & 8 & 3 & 0 \\
\hline Veracruz & $51(10.5)$ & $190(39.1)$ & 449 (92.5) & 57 (11.7) & $10(2.1)$ \\
\hline Yucatán & 35 & 97 & 169 & 6 & 0 \\
\hline Zacatecas & 11 & 21 & 30 & 7 & 0 \\
\hline
\end{tabular}

the most important, hosting 692 species (80.3\%), followed by tropical seasonally dry forest, temperate forest, humid mountain forest and xerophytic scrubland. Regarding the endemic species, the position of the latter two biomes in terms of total species is not altered; however, the top two places are changed because the tropical seasonally dry forest becomes of highest importance ( $n=134$ species, $59.8 \%$ ), whereas second and third position was taken by temperate forest and tropical humid forest, respectively. The values of richness differ significantly among the biomes, both for the total number of species $\left(\chi^{2}=531.16\right.$, d.f. $\left.4, P=0.001\right)$ and the number of endemic species $\left(\chi^{2}=80.55\right.$, d.f. $\left.4, P=0.001\right)$.

When species richness is evaluated by vegetation type, the tropical dry forest occupies first place, both for the total number of species $(52.1 \%)$ and for that of the endemic species (55.8\%); another similarity between both sets of species is the reduced importance of mangrove, savanna and thorn forest (Table 5). Second place in terms of the total number of species is occupied by the tropical evergreen forest $(44.3 \%)$, while for the endemic species this position is held by the oak forest $(24.6 \%)$. The nine vegetation types also showed difference for all lianas species $\left(\chi^{2}=836.92\right.$, d.f. $8, P=0.001)$ and by their number of endemics $\left(\chi^{2}=\right.$ 194.15 , d.f. $8, P=0.001$ ).

Species of lianas are negatively correlated with altitude, with a higher concentration found at lower elevations (Table 6), particularly below $1,500 \mathrm{~m}$ elevation, with a statistically significant correlation, both for the total number of species $(R=-0.98, P=0.001)$ and for endemic species $(R=-0.904$, $P=0.001)$. 
Table 4. Frequency distribution of the number of states occupied by liana species in Mexico.

\begin{tabular}{cccc}
\hline $\begin{array}{c}\text { Number of } \\
\text { states per } \\
\text { species }\end{array}$ & $\begin{array}{c}\text { Total of } \\
\text { species } \\
\text { (endemics) }\end{array}$ & $\begin{array}{c}\text { Number of } \\
\text { states per } \\
\text { species }\end{array}$ & $\begin{array}{c}\text { Total of } \\
\text { species } \\
\text { (endemics) }\end{array}$ \\
\hline $\mathbf{1}$ & $142(57)$ & $\mathbf{1 5}$ & $12(2)$ \\
$\mathbf{2}$ & $109(34)$ & $\mathbf{1 6}$ & $9(1)$ \\
$\mathbf{3}$ & $124(36)$ & $\mathbf{1 7}$ & $7(1)$ \\
$\mathbf{4}$ & $80(14)$ & $\mathbf{1 8}$ & $11(1)$ \\
$\mathbf{5}$ & $65(18)$ & $\mathbf{1 9}$ & $10(2)$ \\
$\mathbf{6}$ & $54(14)$ & $\mathbf{2 0}$ & $9(0)$ \\
$\mathbf{7}$ & $51(11)$ & $\mathbf{2 1}$ & $4(1)$ \\
$\mathbf{8}$ & $35(6)$ & $\mathbf{2 2}$ & $5(0)$ \\
$\mathbf{9}$ & $25(3)$ & $\mathbf{2 3}$ & $4(0)$ \\
$\mathbf{1 0}$ & $25(6)$ & $\mathbf{2 4}$ & $1(0)$ \\
$\mathbf{1 1}$ & $35(11)$ & $\mathbf{2 5}$ & $1(0)$ \\
$\mathbf{1 2}$ & $18(2)$ & $\mathbf{2 6}$ & $1(0)$ \\
$\mathbf{1 3}$ & $9(2)$ & $\mathbf{2 7}$ & $1(0)$ \\
$\mathbf{1 4}$ & $14(2)$ & $\mathbf{3 1}$ & $1(0)$ \\
\hline
\end{tabular}

\section{Discussion}

Taxonomic richness. Fifteen families with the greatest taxonomic diversity include $81 \%$ of the species (Table 1). This figure differs from that documented by Gentry (1991a), who found that 26 families of the Neotropics contained $85 \%$ of

Table 5. Liana species per biome (in bold and cursives) and vegetation types recognized in Mexico. Humid mountain forest biome and xerophytic scrubland biome include only one vegetation type named as the same.

\begin{tabular}{lcc}
\hline Biome and Vegetation & \multicolumn{2}{c}{ Species } \\
\cline { 2 - 3 } & Total (\%) & Endemics (\%) \\
\hline Humid mountain forest & $\mathbf{2 6 0 ( 3 0 . 2 )}$ & $\mathbf{4 7}(\mathbf{2 1 . 0 )}$ \\
Temperate forest & $\mathbf{3 6 1 ( 4 2 . 0 )}$ & $\mathbf{1 1 2 ( 5 0 . 1 )}$ \\
$\quad$ Quercus forest & $168(19.5)$ & $55(24.6)$ \\
$\quad$ Pinus-Quercus forest & $128(15.0)$ & $36(16.1)$ \\
$\quad$ Conipherous forest & $65(7.5)$ & $21(9.4)$ \\
Tropical seasonally dry forest & $\mathbf{4 7 2 ( 5 4 . 8 )}$ & $\mathbf{1 3 4}(\mathbf{5 9 . 8})$ \\
$\quad$ Tropical dry forest & $449(52.1)$ & $125(55.8)$ \\
$\quad$ Thorn forest & $23(2.7)$ & $9(4.0)$ \\
Tropical humid forest & $\mathbf{6 9 2 ( 8 0 . 3 )}$ & $\mathbf{7 4}(\mathbf{3 3 . 1})$ \\
$\quad$ Tropical evergreen forest & $382(44.3)$ & $23(10.3)$ \\
Tropical semi evergreen forest & $310(36.0)$ & $51(22.8)$ \\
Xerophytic scrubland & $\mathbf{9 8 ( 1 1 . 4 )}$ & $\mathbf{4 1}(\mathbf{1 8 . 3 )}$ \\
Other vegetation types & & \\
$\quad$ Riparian forest & $144(16.7)$ & $23(10.3)$ \\
$\quad$ Mangrove & $31(3.6)$ & $2(0.9)$ \\
Savanna & $25(2.9)$ & $3(1.3)$ \\
Secondary vegetation & $309(35.8)$ & $42(18.8)$ \\
\hline
\end{tabular}

Table 6. Relationship between elevation ( $\mathrm{m}$ asl) and number of liana species in Mexico.

\begin{tabular}{lcc}
\hline Altitudinal range & \multicolumn{2}{c}{ Species } \\
\cline { 2 - 3 } & Total (\%) & Endemics (\%) \\
\hline $0-500$ & $596(69.1)$ & $111(49.6)$ \\
$501-1,000$ & $521(60.4)$ & $106(47.3)$ \\
$1,001-1,500$ & $441(51.2)$ & $113(50.4)$ \\
$1,501-2,000$ & $300(34.8)$ & $99(44.2)$ \\
$2,001-2,500$ & $154(17.9)$ & $51(22.8)$ \\
$2,501-3,000$ & $73(8.5)$ & $19(8.5)$ \\
$3,001-3,500$ & $9(1.0)$ & $3(1.3)$ \\
$3,501-4,000$ & $2(0.2)$ & $1(0.4)$ \\
\hline
\end{tabular}

the species that exhibit a scandent habit. Likewise, there is no coincidence at the level of the entire Mexican flora because the most speciose 15 families include $55 \%$ of the total species (Villaseñor, 2003). Families with more species for Mexico as documented by this last author and for the lianas in the present study indicate a marginal similarity because these groups only share Asteraceae, Fabaceae, Rubiaceae, and Solanaceae, although a subsequent modification of the data of Villaseñor (2003) by Juárez-Jaimes et al. (2007) also incorporated Apocynaceae s.l. within the most diverse Mexican families (position 14). These data suggest that the specialization of climbers has centered in particular family lineages (e.g., Apocynaceae, Convolvulaceae, Malpighiaceae, or Passifloraceae). In addition, it is important to consider that in Apocynaceae and Malpighiaceae, the climbing habit is more frequent compared to other growth forms, such as the trees or shrubs (Juárez-Jaimes et al., 2007; Anderson, 2013).

Considering that the 20 most diverse genera in Mexico include $15 \%$ of the species (Villaseñor, 2004), our results are important given that the 18 more diverse liana genera comprise $38.1 \%(n=328)$ of the species (Table 2), despite the fact that the proportion of liana genera with only one species is greater $(50 \%)$ than that of the entire flora $(\mathrm{n}=$ 988; $37 \%$ ). The role of Ipomoea is of the greatest importance, and it is no surprise that it is cited as the second most speciose genus among the Neotropical climbing plants (Gentry, 1991a). If the genera of Table 1 are compared with those of the entire Mexican flora (Villaseñor, 2004), only Solanum and Ipomoea are found, occupying the nineth and tenth positions, respectively.

Geographic distribution. For endemic liana genera, our results are comparable with those of Rzedowski (1991a), who calculated an endemism of $\pm 1 \%$, which is much lower than that obtained for the entire Mexican flora ( $8 \%$ ) by Villaseñor (2004). However, the value of $25.9 \%$ of endemism for liana species is practically half of the percentage that was recently 
obtained for the whole country (Villaseñor and Ortiz, 2014) but is much closer to that estimated by these authors for the climbers $(38 \%)$ or for the vines or woody climbers $(15 \%)$ by Rzedowski (1991a). The causes of this important value of endemism may be found in the role that plant communities (e.g., the tropical deciduous forest) or biomes (e.g., the tropical montane humid forest) have played as "ecological islands", as well as the influence of the climate and edaphic substrate in the life cycle and evolution of plants in Mexico (Rzedowski, 1991a, b).

Another aspect to highlight is that the families and genera that are most notable for their richness of lianas play similar roles in their contribution to endemic and microendemic species (Tables 1,2). The trend for families is explained, in part, by the fact that some of these families are among the most speciose in Mexico, while also presenting noteworthy levels of endemism (Rzedowski, 1991a; Villaseñor, 2003; Juárez-Jaimes et al., 2007): Apocynaceae $47 \%$, Asteraceae 65.9-66 \%, Fabaceae 52-60 \%, and Rubiaceae $49 \%$. A similar explanation can be applied to Ipomoea because it is the tenth most diverse genus in the country (Villaseñor, 2004). An important point to note is the contribution of Apocynaceae, with six genera (Table 2), which present a notable level of restriction within the Mexican territory (Juárez-Jaimes et al., 2007): Dictyanthus (87\%), Gonolobus (49\%), Mandevilla (47\%), Marsdenia (53\%), Matelea (60\%), and Metastelma (52\%).

The richness, number of endemics and of the microendemic species that were detected among the lianas of Mexico indicate a very clear inequality among the Mexican states; this relationship is maintained for the seven most important states, regardless of their different sizes (Table 3). One factor that explains these relationships, both for the total number of liana species and for the endemic species, is the proximity of the states (Ibarra-Manríquez et al., 2015). Consequently, the floristic importance at the national level of the states of Chiapas, Guerrero, Jalisco, Michoacán, Oaxaca, and Veracruz is ratified by our results, which support similar conclusions by studies that were conducted on different groups of plants (e.g., Villaseñor et al., 1998; Espejo-Serna et al., 2004a; Juárez-Jaimes et al., 2007; Alcántar-Mejía et al., 2012) or the entire flora (Rzedowski, 1991a, b; Villaseñor, 2003; Villaseñor and Ortiz, 2014).

The lianas found in only one state (Table 4) present much lower values for the total number of species $(16.5 \%)$ and endemic species $(26 \%)$ than those obtained by Villaseñor (2003) for the entire Mexican flora, with $33 \%(n=8,228$ of 24,626 species), and $43 \%(n=5,566$ of 12,900$)$, respectively. However, a direct comparison cannot be made because the figures that were presented by this author include subspecific categories. In addition, as indicated by Villaseñor (2003), these results must be treated with caution as they may be affected by collection bias, thus highlighting the need for a future floristic study that is directed towards a more exact characterization of the geographic distribution of these rare species.

Biomes, vegetation types and altitude. At the level of biomes, the tropical forests have proved to be the most important for liana species, a global pattern that has been indicated in previous studies (Gentry, 1982, 1991a, b; Schnitzer and Bongers, 2002; Schnitzer, 2005). The role of the tropical humid forest is particularly remarkable for its number of species, while the tropical seasonally dry forest stands out for its endemics (Table 5). Contrasting results are seen at the level of the entire flora of Mexico (Villaseñor and Ortiz, 2014) because the temperate forest has the greatest importance both at the level of total richness and of endemic species; in fact, in this latter group of taxa, the tropical humid forest is the least important. These data affirm the significance of conducting particular biogeographic studies for the different growth forms present in Mexico because some can present patterns that are discordant with those that are obtained for the entire flora.

In terms of the hosted liana species, the vegetation types that are recognized in Mexico are of uneven importance (Table 5). In this sense, it is notable that first place for the total number of species and of endemic species is occupied by the tropical deciduous forest, ratifying the ecological and floristic importance of this vegetation type at the global (Olson and Dinerstein, 2002), continental (Lott and Atkinson, 2006) and national scales (e.g., Trejo and Dirzo, 2002; CuéBär et al., 2006a, b; Juárez-Jaimes et al., 2007; Rzedowski and Calderón de Rzedowski, 2013). The importance of the oak forest for endemic liana species was not expected because this vegetation type had been considered important for other growth forms, especially herbaceous plants (e.g., Rzedowski, 1978).

However, our results agree with those of Gentry (1991a), Bhattarai and Vetaas (2003) and Jiménez-Castillo et al. (2007) in terms of the negative relationship between the richness of lianas and the altitude. Additional evidence is found in Jiménez-Castillo et al. (2007), who analyzed the data for the lianas in a gradient of tropical vegetation in Mexico and detected the same statistically significant trend. One factor that may explain this result is the sensitivity of lianas to temperatures below the freezing point, which affect their vascular system and impede an efficient transport of water from the root to the crown (Gentry, 1991b; Schnitzer and Bongers, 2002; Schnitzer, 2005). However, the sensitivity of lianas to this factor must be explored in greater detail because other factors that change with altitude could also affect these plants; for example, the total annual precipitation, the seasonality of precipitation or the relative humidity of the air. This last factor has a direct effect on the hydric balance of the plants and the energy availability (Schnitzer and Bongers, 2002; Schnitzer, 2005). Furthermore, it should be considered that lianas in Mexico are by no means scarce 
in temperate forests (Table 5), justifying the future investigation of the influence of frost and other climatic factors on liana physiology and ecology.

\section{Conclusions}

Mexican lianas present patterns of diversity and distribution that must be considered when defining strategies that are directed toward their conservation. The coincidence in the richness, endemism and microendemic taxa at the state level is of particular value, highlighting the relevance of these attributes in the states of Chiapas, Oaxaca, Guerrero, Jalisco and Michoacán (Table 3), where the establishment of Protected Areas is a urgent priority; the same conclusion can be invoked if we analyze the patterns of richness and endemism for the whole flora (Villaseñor, 2003; Villaseñor and Ortíz, 2014). These reserves should have the consent of the different social and political stakeholders that are involved, as well as financial income to guarantee their long-term functioning. From the results that were obtained for the lianas, it is clear that these reserves must include important areas of tropical and temperate forest within the vegetation types that are under protection, especially the tropical deciduous forest, tropical montane cloud forest and oak forest. The high beta diversity that is exhibited by lianas is concordant with that exhibited by the flora (Villaseñor, 2003; PérezGarcía et al., 2005; Trejo, 2005) and fauna (Rodríguez et al., 2003; Flores-Villela et al., 2005), and their complementarity should be considered during these conservation exercises, especially in terms of the endemic species.

The data from the present study indicate that lianas play a fundamental role in the taxonomic diversity of the flora of Mexico, which is one of the greatest worldwide. Botanical exploration is an important task that must still be completed, particularly for those biological groups underrepresented in plant inventories and collections mainly by the difficulties inherent in their biological form, such as lianas, in which many species represent microendemics, that are currently being discovered and named (e.g. Cuevas-Guzmán and NúñezLópez, 2015; Linares, 2015). However, Ibarra-Manríquez et al. (2015) indicated that the conservation status of lianas in Mexico is of concern. For this reason, it is hoped that our contribution can promote the development of studies that further the knowledge of the biogeography, ecology and systematic of the lianas in this country, providing broader information with which to prioritize efforts to ensure the future conservation of lianas in the ecosystems that they occupy.

\section{Acknowledgments}

This study formed part of the postdoctoral research project of Pablo Carrillo-Reyes "Diversity and biogeography of the Mexican lianas", from 2011-2012, which was funded by the Consejo Técnico de la Investigación Científica (oficio CJIC/
CTIC/2318/2010) of the Universidad Nacional Autónoma de México (UNAM). Francisco Javier Rendón-Sandoval was a Master's student in the Posgrado de Ciencias Biológicas of UNAM, for which he gratefully received a grant from the Consejo Nacional de Ciencia y Tecnología (CONACyT). Thanks to Heberto Ferreira Medina, Alberto Valencia García and Atzimba López Maldonado for their support with data base and computer programs. Finally, we thank José Luis Villaseñor Ríos, Luis Enrique Eguiarte Frunz and an anonymous reviewer for important comments on the manuscript.

\section{Literature cited}

Alcántar-Mejía J., Carranza-González E., Cuevas-García G. and Cuevas-García E. 2012. Distribución geográfica y ecológica de Ipomoea (Convolvulaceae) en el estado de Michoacán, México. Revista Mexicana de Biodiversidad 83:731-741.

Alvarado-Cárdenas L.O. 2004. Apocynaceae. Flora del Valle de Tehuacán-Cuicatlán, Fascículo 38. Instituto de Biología, Universidad Nacional Autónoma de México, México.

Anderson W.R. 2013. Origins of Mexican Malpighiaceae. Acta Botanica Mexicana 104:107-156.

Bhattarai K.R. and Vetaas O.R. 2003. Variation in plant species richness of different life forms along a subtropical elevation gradient in the Himalayas, east Nepal. Global Ecology and Biogeography 12:327-340.

Bongers F., Schnitzer S.A. and Traoré D. 2002. The importance of lianas and consequences for forest management in West Africa. Bioterre, Revue International des Sciences de la Vie et de la Terre $\mathbf{N}^{\circ}$ spécial:59-70.

Borhidi A. 2006. Rubiáceas de México. Academia de Ciencias de Hungría, Budapest.

Calderón de Rzedowski G., Rzedowski J. and MacDougal J.M. 2004. Passifloraceae. Flora del Bajío y de Regiones Adyacentes Fascículo 121. Instituto de Ecología A.C., Pátzcuaro.

Cué-Bär E.M., Villaseñor J.L., Arredondo-Amezcua L., CornejoTenorio G. and Ibarra-Manríquez G. 2006a. La flora arbórea de Michoacán, México. Boletín de la Sociedad Botánica de México 78:47-81.

Cué-Bär E.M., Villaseñor J.L., Morrone J.J., and Ibarra-Manríquez G. 2006b. Identifying priority areas for conservation in Mexican tropical deciduous forest based on tree species. Interciencia 31:712-719.

Cuevas-Guzmán R. and Núñez-López N.M. 2015. Solanum edmundoi (Solanaceae), una especie nueva de bejuco con aguijones del occidente de México. Revista Mexicana de Biodiversidad 86:293-297.

Espejo-Serna A., López-Ferrari A. R. and Salgado U.I. 2004a. A current estimate of Angiosperm diversity in Mexico. Taxon 53:127-130.

Espejo-Serna A., López-Ferrari A.R., Ramírez-Morillo I., Holst B.K., Luther H.E. and Till W. 2004b. Checklist of Mexican Bromeliaceae with notes on species distribution and levels of endemism. Selbyana 25:33-86.

Ferrusquía-Villafranca I. 1993. Geology of Mexico: a synopsis. In: Ramamoorthy T.P., Bye R., Lot A. and Fa J. Eds. Biological Diversity of Mexico: Origins and Distribution, pp. 3-108, Oxford University Press, Oxford. 
Flores-Villela O., Ochoa-Ochoa L. and Moreno C.E. 2005. Variación latitudinal y longitudinal de la riqueza de especies y la diversidad beta de la herpetofauna mexicana. In: Halffter G., Soberón J., Koleff P. and Melic A. Eds. Sobre Diversidad Biológica: el Significado de las Diversidades Alfa, Beta y Gama, pp. 143-151, Monografías Tercer Milenio, vol. 4, Sociedad Entomológica Aragonesa, Comisión Nacional para el Conocimiento y Uso de la Biodiversidad, Grupo Diversitas-México y Consejo Nacional de Ciencia y Tecnología, Zaragoza.

Gallagher R.V. and Leishman M.R. 2012. A global analysis of trait variation and evolution in climbing plants. Journal of Biogeography 39:1757-1771.

Gentry A.H. 1982. Patterns of Neotropical plant species diversity. Evolutionary Biology 15:1-84.

Gentry A.H. 1991a. The distribution and evolution of climbing plants. In: Putz F.E. and Mooney H.A. Eds. The Biology of Vines, pp. 3-52, Cambridge University Press, Cambridge.

Gentry A.H. 1991b. Breeding and dispersal systems of lianas. In: Putz F.E. and Mooney H.A. Eds. The Biology of Vines, pp. 393423, Cambridge University Press, Cambridge.

Gentry A.H and Dodson C.H. 1987.Contribution of nontrees to species richness of a tropical rain forest. Biotropica 19:149-156.

González-Espinosa M., Meave J.A., Lorea-Hernández F.G., Ibarra-Manríquez G. and Newton A.C. Eds. 2011. The Red List of Mexican Cloud Forest Trees. Fauna and Flora International, Cambridge.

Hágsater E., Soto-Arenas M.A., Salazar-Chávez G.A., JiménezMachorro R., López-Rosas M.A. and Dressler R.L. 2005. Las Orquídeas de México. Instituto Chinoín, México D.F.

Ibarra-Manríquez G., Villaseñor J.L. and Durán R. 1995. Riqueza y endemismo del componente arbóreo de la Península de Yucatán, México. Boletín de la Sociedad Botánica de México 57:49-77.

Ibarra-Manríquez G. and Sinaca S. 1995. Lista florística comentada de la Estación de Biología Tropical "Los Tuxtlas", Veracruz, México. Revista de Biología Tropical 43:75-115.

Ibarra-Manríquez G. and Sinaca S. 1996a. Estación de Biología Tropical "Los Tuxtlas", Veracruz, México: lista florística comentada (Mimosaceae a Verbenaceae). Revista de Biología Tropical 44:41-60.

Ibarra-Manríquez G. and Sinaca S. 1996b. Estación de Biología Tropical "Los Tuxtlas", Veracruz, México: lista florística comentada (Violaceae a Zingiberaceae). Revista de Biología Tropical 44:427-447.

Ibarra-Manríquez G., Carrillo-Reyes P., Rendón-Sandoval F. J. and Cornejo-Tenorio G. 2015. Diversity and distribution of lianas in Mexico. In: Schnitzer S.A., Bongers F., Burnham R.J. y Putz F. Eds. The Ecology of Lianas, pp. 93-105. John Wiley \& Sons, LTD., Oxford.

Jiménez-Castillo M., Wiser S.K. and Lusk C.H. 2007. Elevational parallels of latitudinal variation in the proportion of lianas in woody floras. Journal of Biogeography 34:163-168.

Juárez-Jaimes V. and Lozada L. 2003. Asclepiadaceae. Flora del Valle de Tehuacán-Cuicatlán, Fascículo 37. Instituto de Biología, Universidad Nacional Autónoma de México, México.

Juárez-Jaimes V., Alvarado-Cárdenas L.O. and Villaseñor J.L. 2007. La familia Apocynaceae sensu lato en México: diversidad y distribución. Revista Mexicana de Biodiversidad 78:459482.

Kottek M., Grieser J., Beck C., Rudolf B. and Rubel F. 2006. World Map of the Köppen-Geiger climate classification updated. $M e$ - teorologische Zeitschrift 15:259-263.

Letcher S.G. and Chazdon R.L. 2012. Life history traits of lianas during tropical forest succession. Biotropica 44:720-727.

Linares J.L. 2015. Nuevas especies de Machaerium (Leguminosae: Papilionoideae: Dalbergiae) en México y Centroamérica. Journal of the Botanical Research Institute of Texas 9:49-61.

Lott E.J. 2002. Lista anotada de las plantas vasculares de Chamela-Cuixmala. In: Noguera F.A., Vega R.J.H, García-Aldrete A.N. and Quesada-Avendaño M. Eds. Historia Natural de Chamela, pp. 99-136, Instituto de Biología, Universidad Nacional Autónoma de México, México D.F.

Lott E.J., Bullock S.H. and Solís-Magallanes A.J. 1987. Floristic diversity and structure of upland and arroyo forest of coastal Jalisco. Biotropica 19:228-235.

Lott E.J. and Atkinson T.H. 2006. Mexican and Central American seasonally dry tropical forests: Chamela-Cuixmala, Jalisco, as a focal point for comparison. In: Pennington T., Lewis G.P. and Ratter J.A. Eds. Neotropical Savannas and Seasonally Dry Forests, Plant Diversity, Biogeography, and Conservation, pp. 315-342, The Systematics Association Special Volume Series 69. CRC Press, Taylor and Francis Group, Boca Raton.

Martínez-Cruz J., Méndez-Toribio M., Cortés-Flores J., CobaPérez P., Cornejo-Tenorio G. and Ibarra-Manríquez G. 2013. Estructura y diversidad de los bosques estacionales desaparecidos por la construcción de la presa Gral. Francisco J. Múgica, en la Depresión del Balsas, Michoacán, México. Revista Mexicana de Biodiversidad 84:1216-1234.

Meave J.A., Romero-Romero M.A., Valle-Doménech A., RincónGutiérrez A., Martínez E. and Ramos C.H. 2008. Plant diversity assessment in the Yaxchilán Natural Monument, Chiapas, Mexico. Boletín de la Sociedad Botánica de México 83:53-76.

Miranda F. and Hernández-X. E. 1963. Los tipos de vegetación de México y su clasificación. Boletín de la Sociedad Botánica de México 28:29-179.

Olson D.M. and Dinerstein E. 2002. The global 200: priority ecoregions for global conservation. Annals of the Missouri Botanical Garden 89:199-224.

Ortega J.F. and Ortega R.V. 1997. Aristolochiaceae. Flora de Veracruz, Fascículo 99. Instituto de Ecología A.C., Xalapa.

Padilla-Velarde E., Cuevas-Guzmán R., Ibarra-Manríquez G. y Moreno-Gómez S. 2006. Riqueza y biogeografía de la flora arbórea del estado de Colima, México. Revista Mexicana de Biodiversidad 77:271-295.

Pérez-Cueto E. 1995. Menispermaceae. Flora de Veracruz, Fascículo 87. Instituto de Ecología A.C., Xalapa.

Pérez-García E.A., Meave J.A. and Gallardo-Cruz J.A. 2005. Diversidad $\beta$ y diferenciación florística en un paisaje complejo del trópico estacionalmente seco del sur de México. In: Halffter G., Soberón J., Koleff P. and Melic A. Eds. Sobre Diversidad Biológica: el Significado de las Diversidades Alfa, Beta y Gama, pp. 123-142, Monografías Tercer Milenio, vol. 4, Sociedad Entomológica Aragonesa, Comisión Nacional para el Conocimiento y Uso de la Biodiversidad, Grupo Diversitas-México y Consejo Nacional de Ciencia y Tecnología, Zaragoza.

Pineda-García F., Arredondo-Amezcua L. and Ibarra-Manríquez G. 2007. Riqueza y diversidad de especies leñosas del bosque tropical caducifolio de El Tarimo, Cuenca del Balsas, Guerrero. Revista Mexicana de Biodiversidad 78:129-139.

Rejmánek M. 1996. Species richness and resistance to invasions. 
In: Orians G., Dirzo R. and Cushman J.H. Eds. Biodiversity and Ecosystem Processes in Tropical Forests, pp.153-172, SpringerVerlag, Berlin.

Ricker M., Hernández H.M., Sousa M. and Ochoterena H. 2013. Tree and tree-like species of Mexico: Asteraceae, Leguminosae and Rubiaceae. Revista Mexicana de Biodiversidad 84:439470.

Rodríguez P., Soberón J. and Arita H.T. 2003. El componente beta de la diversidad de mamíferos de México. Acta Zoologica Mexicana 89:241-259.

Rzedowski J. 1978. Vegetación de México. Ed. Limusa, México D.F.

Rzedowski J. 1991a. Diversidad y orígenes de la flora fanerogámica de México. Acta Botanica Mexicana 14:3-21.

Rzedowski J. 1991b. El endemismo de la flora fanerogámica de México, una apreciación analítica preliminar. Acta Botanica Mexicana 15:47-64.

Rzedowski J. and Calderón de Rzedowski G. 1993. Bignoniaceae. Flora del Bajío y de Regiones Adyacentes, Fascículo 22. Instituto de Ecología A.C., Pátzcuaro.

Rzedowski J. and Calderón de Rzedowski G. 2013. Datos para la apreciación de la flora fanerogámica del bosque tropical caducifolio de México. Acta Botanica Mexicana 102:1-23.

Schnitzer S.A. 2005. A mechanistic explanation for global patterns of liana abundance and distribution. American Naturalist 166:262-276.

Schnitzer S.A. and Bongers F. 2002. The ecology of lianas and their role in forests. Trends in Ecology and Evolution 17:223-230.

Schnitzer S.A., Kuzee M.E. and Bongers F. 2005. Disentangling above- and below-ground competition between lianas and trees in tropical forest. Journal of Ecology 93:1115-1125.

Solórzano S., Ibarra-Manríquez G. and Oyama K. 2002. Liana diversity and reproductive attributes in two tropical forests in
Mexico. Biodiversity and Conservation 11:197-212.

Soto-Arenas M. A., Hágsater E., Jiménez-Machorro R. and Solano-Gómez R. 2007. Orquídeas de México. Herbario AMOInstituto Chinoín, A.C. y Centro Interdisciplinario de Investigación para el Desarrollo Integral Regional-Unidad-Oaxaca, Instituto Politécnico Nacional. Informe final SNIB-CONABIO proyecto No. P107. México D.F.

Trejo I. 2005. Análisis de la diversidad de la selva baja caducifolia en México. In: Halffter G., Soberón J., Koleff P. and Melic A. Eds. Sobre Diversidad Biológica: el Significado de las Diversidades Alfa, Beta y Gama, pp. 111-122, Monografías Tercer Milenio, vol. 4, Sociedad Entomológica Aragonesa, Comisión Nacional para el Conocimiento y Uso de la Biodiversidad, Grupo Diversitas-México y Consejo Nacional de Ciencia y Tecnología, Zaragoza.

Trejo I. and Dirzo R. 2002. Floristic diversity of Mexican seasonally dry tropical forests. Biodiversity and Conservation 11:2063-2048.

Tropicos. 2014. Tropicos.org. Missouri Botanical Garden. <http:// www.tropicos.org > (4 Septiembre 2014).

Villaseñor J.L. 2003. Diversidad y distribución de las Magnoliophyta de México. Interciencia 28:160-167.

Villaseñor J.L. 2004. Los géneros de plantas vasculares de la flora de México. Boletín de la Sociedad Botánica de México 75:105135.

Villaseñor J.L., Ibarra-Manríquez G. and Ocaña D. 1998. Strategies for the conservation of Asteraceae in Mexico. Conservation Biology 12: 1066-1075.

Villaseñor J.L. and Ortiz E. 2014. Biodiversidad de las plantas con flores (División Magnoliophyta) en México. Revista Mexicana de Biodiversidad Supl. 85:S134-S142.

Wyka T.P., Oleksyn J., Karolewski P. and Schnitzer S.A. 2013. Phenotypic correlates of the lianescent growth form: a review. Annals of Botany 112:1667-1681.

Received: May 2nd, 2014

Accepted: Septembrer 15th, 2014 
Appendix 1.Floristic and taxonomic literature revised in the present study.

Acevedo-Rodríguez P. 1997. Two new species of Serjania section Serjania (Sapindaceae). Brittonia 49:498-502.

Acosta S., Flores A., Saynes A., Aguilar R. and Manzanero G. 2003. Vegetación y flora de una zona semiárida de la cuenca alta del Río Tehuantepec, Oaxaca, México. Polibotánica 16:125-152.

Alcántara A.O. and Luna I.V. 1997. Florística y análisis biogeográfico del bosque mesófilo de montaña de Tenango de Doria, Hidalgo, México. Anales del Instituto de Biología de la Universidad Nacional Autónoma de México. Serie Botánica 68:57-106.

Almeda F. 2001. Melastomataceae. Flora de Nicaragua. Monographs on Systematic Botany from the Missouri Botanical Garden, Vol. 85, pp. 1339-1418. Missouri Botanical Garden Press, St. Luis.

Alvarado-Cárdenas L.O. 2007. Passifloraceae. Flora del Valle de Tehuacán-Cuicatlán, Fascículo 48. Instituto de Biología, Universidad Nacional Autónoma de México, México D.F.

Anderson C. 1997. Monograph of Stigmaphyllon (Malpighiaceae). Monographs on Systematic Botany from the Missouri Botanical Garden, Vol. 51, 1-313 Missouri Botanical Garden Press, St. Luis.

Anderson C. 2001. Novelties in Mascagnia (Malpighiaceae). Brittonia 53:405-415.

Anderson W.R. 1987. Notes on Neotropical Malpighiaceae-II. Contributions from the University of Michigan Herbarium 16:55-108.

Anderson W.R. 2001. Malpighiaceae. Flora de Nicaragua. Monographs on Systematic Botany from the Missouri Botanical Garden, Vol. 85, 1339-1418. Missouri Botanical Garden Press, St. Luis.

Anderson W.R. 2006a.A new species of Callaeum (Malpighiaceae) from Puebla, Mexico. Acta Botanica Mexicana 74:179-183.

Anderson W.R. 2006b. Eight segregates from the Neotropical genus Mascagnia (Malpighiaceae). Novon 16:168-204.

Anderson W.R. and Corso S. 2007. Psychopterys, a new genus of Malpighiaceae from Mexico and Central America. Contributions from the University of Michigan Herbarium 25:113-135.

Anderson W.R. and Davis C.C. 2007. Generic adjustments in Neotropical Malpighiaceae. Contributions from the University of Michigan Herbarium 25:137-166.

Andress M.E. and Hansen B.F. 2007. Pinochia, a new genus of Apocynaceae, Apocynoideae from the Greather Antilles, Mexico and Central America. Edinburgh Journal of Botany 64:269-274.

Arias S., Gama S. and Guzmán L.U. 1997. Cactaceae. Flora del Valle de Tehuacán-Cuicatlán, Fascículo14. Instituto de Biología, Universidad Nacional Autónoma de México, México D.F.

Austin D.F. 2001. Convolvulaceae. Flora de Nicaragua. Monographs on Systematic Botany from the Missouri Botanical Garden, Vol. 85, pp. 653-679. Missouri Botanical Garden Press, St. Luis.

Austin D.F., McDonald J.A. and Murguía-Sánchez G. 2011.Convolvulaceae. In Davidse G., Sousa S., Knapp M. and Chiang F. Eds. Flora Mesoamericana Vol.4, Parte 1, pp. 1-109. Instituto de Biología, Universidad Nacional Autónoma de México, Missouri Botanical Garden and The Natural History Museum (London), México D.F.

Barrie F.R. 2003. Seven new species and one new variety of Valeriana (Valerianaceae) from Mexico. Acta Botanica Mexicana 62:31-64.

Barrie F.R. 2001. Valerianaceae. Flora de Nicaragua. Monographs on Systematic Botany from the Missouri Botanical Garden, Vol. 85, pp. 2495-2497. Missouri Botanical Garden Press, St. Luis.

Bedell H.G. 1980. A taxonomic and morphological re-evaluation of Stegnospermaceae (Caryophyllales). Systematic Botany 5:419-431.

Bernardello L.M. and Hunziker A.T. 1987. A synoptical revision of Solandra (Solanaceae). Nordic Journal of Botany 7:639-652.

Borhidi A. and Diego-Pérez N. 2008. Coussareae, Gardenieae, Hedyotideae, Mussaendeae, Naucleae, Rondeletieae (Rubiaceae). Flora de Guerrero, Fascículo 35. Facultad de Ciencias, Universidad Nacional Autónoma de México, México D.F.

Borsch T. 2001. Amaranthaceae. Flora de Nicaragua. Monographs on Systematic Botany from the Missouri Botanical Garden, Vol. 85, pp. 56-83. Missouri Botanical Garden Press, St. Luis.

Burger W. and Barringer K. 2000. Flora Costaricensis: Schlegeliaceae. Fieldiana Botany 41:69-77.

Calderón de Rzedowski G and Rzedowski J. 1994. Smilacaceae. Flora del Bajío y de regiones adyacentes, Fascículo 26. Instituto de Ecología A.C., Pátzcuaro.

Calderón de Rzedowski G. 1997. Basellaceae. Flora del Bajío y de regiones adyacentes, Fascículo 59. Instituto de Ecología A.C., Pátzcuaro.

Calderón de Rzedowski G. 1999. Menispermaceae. Flora del Bajío y de regiones adyacentes, Fascículo 72 . Instituto de Ecología, A.C., Centro Regional del Bajío. Pátzcuaro.

Calderón de Rzedowski G and Rzedowski J. 2006. Sapindaceae. Flora del Bajío y de regiones adyacentes, Fascículo 142. Instituto de Ecología A.C., Pátzcuaro.

Calónico-Soto J. 2011. Sapindaceae. Flora del Valle de Tehuacán-Cuicatlán, Fascículo 86. Instituto de Biología, Universidad Nacional Autónoma de México, México D.F.

Campos A., Kelly L.M. and Delgado A. 2004. Bejucos y otras trepadoras de la Estación de Biología Tropical Los Tuxtlas, Veracruz, México. Cuadernos del Instituto de Biología 36. Instituto de Biología, Universidad Nacional Autónoma de México y The New York Botanical Garden, México D.F. 
Appendix 1. Continuation.

Carranza E. 2001. Hippocrateaceae. Flora del Bajío y de regiones adyacentes, Fascículo 98. Instituto de Ecología A.C., Pátzcuaro.

Carranza E. 2007. Convolvulaceae I. Flora del Bajío y de regiones adyacentes, Fascículo 151. Instituto de Ecología A.C., Pátzcuaro.

Carranza E. 2008. Diversidad del género Ipomoea L. (Convolvulaceae) en el estado de Michoacán, México. Flora del Bajío y de regiones adyacentes, Fascículo complementario 23. Instituto de Ecología A.C., Pátzcuaro.

Carrillo-Reyes P. 2009. Menispermaceae. Flora del Valle de Tehuacán-Cuicatlán, Fascículo 70. Instituto de Biología, Universidad Nacional Autónoma de México, México D.F.

Carrillo-Reyes P., Sahagún-Godínez E. and Ibarra-Manríquez G. 2013. The genus Disciphania (Menispermaceae:Tinosporoideae, Tinosporeae) in Mexico. Brittonia 65:439-451.

Cartujano S., Zamudio S., Alcántara O. and Luna I. 2002. El bosque mesófilo de montaña en el municipio de Landa de Matamoros, Querétaro. Boletín de la Sociedad Botánica de México 70:13-43.

Castelo E. 2006. Combretaceae. Flora de Guerrero, Fascículo 28. Facultad de Ciencias, Universidad Nacional Autónoma de México, México D.F.

Castillejos C. and Solano E. 2008. Polygonaceae. Flora del Bajío y de regiones adyacentes, Fascículo 153. Instituto de Ecología A.C., Pátzcuaro.

Castillo-Campos G. 1994. Solanum guamuchilense sp. nov. (Solanaceae, Solaneae) del estado de Nayarit, México. Acta Botanica Mexicana 27:33-37.

Castillo-Campos G., Robles R.G. and Medina M.E. 2003. Flora y vegetación de la sierra Cruz Tetela, Veracruz, México. Polibotánica 15:41-87.

Castillo-Campos G. and Medina M.E. 2005. Hippocrateaceae. Flora de Veracruz, Fascículo 137. Instituto de Ecología A.C., Xalapa.

Castillo-Campos G., Medina M.E., Dávila P.D. and Zavala J.A. 2005. Contribución al conocimiento del endemismo de la flora vascular en Veracruz, México. Acta Botanica Mexicana 73:19-57.

Castillo-Campos G., Dávila-Aranda P. and Zavala-Hurtado J.A. 2007. La selva baja caducifolia en una corriente de lava volcánica en el centro de Veracruz: lista florística de la flora vascular. Boletín de la Sociedad Botánica de México 80:77-104.

Cook K. 2001. Aristolochiaceae. Flora de Nicaragua. Monographs on Systematic Botany from the Missouri Botanical Garden, Vol. 85, pp. 229-233. Missouri Botanical Garden Press, St. Luis.

Cornejo X. and Hiltis H. 2012. Capparaceae. Flora de Jalisco y áreas colindantes, Fascículos 25. Universidad de Guadalajara, Zapopan.

Cornejo-Tenorio G. and Ibarra-Manríquez G. 2011. Diversidad y distribución del género Salvia (Lamiaceae) en Michoacán, México. Revista Mexicana de Biodiversidad 82:1279-1296.

Cristóbal C.L. 2001. Sterculiaceae. Flora de Nicaragua. Monographs on Systematic Botany from the Missouri Botanical Garden, Vol. 85, 2428-2437. Missouri Botanical Garden Press, St. Luis.

Croat T.B. 1976. Notes on Central and South American Cissus (Vitaceae). Annals of the Missouri Botanical Garden 63:358-362.

Cruz R. and Sousa M. 2004. Nissolia ruddiae (Leguminosae, Papilionoideae), una especie nueva de la cuenca del Balsas, México. Acta Botanica Mexicana 68:65-71.

Cuatrecasas J. and Croat T.B. 1980. Flora de Panama part VI, Family 93. Malpighiaceae. Annals of the Missouri Botanical Garden 67:851-945.

Daniel T.F. 1992. Acanthaceae: Mendoncioideae of Mexico. Acta Botanica Mexicana 17:53-60.

Daniel T.F. 1995. Acanthaceae. Flora of Chiapas, Part 4. California Academy of Sciences, San Frascisco.

Daniel T.F. 2010. Catalog of Guatemalan Acanthaceae: taxonomy, ecology, and conservation. Proceedings of the California Academy of Sciences 61:289-377.

D'Arcy W. 2001. Solanaceae. Flora de Nicaragua. Monographs on Systematic Botany from the Missouri Botanical Garden, Vol. 85, pp. 2376-2426. Missouri Botanical Garden Press, St. Luis.

Dávila P., Villaseñor J.L., Medina R., Ramírez A., Salinas A., Sánchez-Ken J. and Tenorio P. 1993. Listados Florísticos de México, Flora del Valle de Tehuacán-Cuicatlán, Fascículo 10. Instituto de Biología, Universidad Nacional Autónoma de México, México D.F.

De Santiago J.R. 2011. Polemoniaceae. Flora de Guerrero, Fascículo 46. Facultad de Ciencias, Universidad Nacional Autónoma de México, México D.F.

Delgadillo-Rodríguez J. and Macías-Rodríguez M.A. 2002. Componente florístico del Desierto de San Felipe, Baja California, México. Boletín de la Sociedad Botánica de México 70:45-65.

Diego N. and Pérez L. 2006. Thoreauea guerrerensis (Apocynaceae, Apocynoideae), una nueva especie de Guerrero, México. Novon: a journal botanical nomenclature 16:332-335.

Diego-Pérez N. 2004. Apocynaceae. Flora de Guerrero, Fascículo 20. Facultad de Ciencias, Universidad Nacional Autónoma de México, México D.F.

Diego-Pérez N. 2011. Sterculiaceae. Flora de Guerrero, Fascículo 45. Facultad de Ciencias, Universidad Nacional Autónoma de México, México D.F. 
Appendix 1. Continuation.

Dillon M.O., Harriman N.A., Turner B.L., Keeley S.C., Keil D.J., Stuessy T.F., Sundberg S., Jansen R.K. and Spooner D.M. 2001. Asteraceae. Flora de Nicaragua. Monographs on Systematic Botany from the Missouri Botanical Garden, Vol. 85, pp. 271393. Missouri Botanical Garden Press, St. Luis.

Dorado O. 1997. Inventario florístico de la Sierra de Huautla, Morelos. Informe final SNIB-CONABIO proyecto No. B054. Universidad Autónoma del Estado de Morelos, México.

Durán R., Trejo-Torres J.C. and Ibarra-Manríquez G. 1998. Endemic phytotaxa of the Peninsula of Yucatán. Harvard Papers in Botany 3:263-314.

Durán-Espinosa C. 1997. Dichapetalaceae. Flora de Veracruz, Fascículo 101. Instituto de Ecología A.C., Xalapa.

Durán-Espinosa C.M. 2003. Gelsemiaceae. Flora de Veracruz, Fascículo 133. Instituto de Ecología A.C., Xalapa.

Enríquez E.D., Koch S.D. and González-Elizondo M.S. 2003. Flora y vegetación de la Sierra de Órganos, municipio de Sombrerete, Zacatecas, México. Acta Botanica Mexicana 64:45-89.

Escalante S. 2000. Flora del Jardín Botánico. In: Sánchez G. and Islebe G.A. Eds. El Jardín Botánico Dr. Alfredo Barrera Marín, pp. 27-47, Comisión Nacional para el Conocimiento y Uso de la Biodiversidad y El Colegio de la Frontera Sur, México D.F.

Espejo A. 1992. Hernandiaceae. Flora de Veracruz, Fascículo 67. Instituto de Ecología A.C., Xalapa.

Espinosa-Jiménez J.A., Pérez-Farrera M.A. and Martínez-Camilo R. 2011. Inventario florístico del parque nacional Cañón del Sumidero, Chiapas, México. Boletín de la Sociedad Botánica de México 89:37-82.

Estrada C.E., Villarreal J.A. and Jurado E. 2005. Leguminosas del norte del estado de Nuevo León, México. Acta Botanica Mexicana 73:1-18.

Estrada-Castillón E. and Villarreal-Quintanilla J.A. 2010. Flora del centro del estado de Chihuahua, México. Acta Botanica Mexicana 92:51-118.

Farrera O. and Bye R. 2011.El género Solandra Sw. (Solanaceae) en Mesoamérica. Lacandonia 5:43-80.

Fay J.J. 1980. Nyctaginaceae. Flora de Veracruz, Fascículo 13. Instituto de Ecología A.C., Xalapa.

Felger R. 1999. The Flora of the Cañón de Nacapule: A desert-bounded tropical canyon near Guaymas, Sonora, Mexico. Proceedings of the San Diego Society of Natural History 35:1-42.

Felger R.S., Austin D.F., Van Devender T.R., Sánchez-Escalante J.J. and Costea M. 2012.Convolvulaceae of Sonora, Mexico. I. Convolvulus, Cressa, Dichondra, Evolvulus, Ipomoea, Jacquemontia, Merremia, and Operculina. Journal of the Botanical Research Institute of Texas 6:459-527.

Fernández R. 1996. Rhamnaceae. Flora del Bajío y de regiones adyacentes, Fascículo 43. Instituto de Ecología, A.C., Pátzcuaro.

Fernández R., Rodríguez J.C., Arreguín S.M.L. and Rodríguez J.A. 1998. Listado florístico de la cuenca del Río Balsas, México. Polibotánica 9:1-151.

Fernández R. 1986. Rhamnaceae. Flora de Veracruz, Fascículo 50. Instituto de Ecología A.C., Xalapa.

Ferrufino A.L. and Gómez-Laurito J. 2004. Estudio morfológico de Smilax L. (Smilacaceae) en Costa Rica, con implicaciones sistemáticas. Lankesteriana 4:5-36.

Fonseca R.M. 1995. Hippocrateaceae. Flora de Guerrero, Fascículo 3. Facultad de Ciencias, Universidad Nacional Autónoma de México, México D.F.

Fonseca R.M. 2005. Connaraceae y Resedaceae. Flora de Guerrero, Fascículo 23. Facultad de Ciencias, Universidad Nacional Autónoma de México, México D.F.

Fonseca R.M. and Ortíz M.F. 2007. Violaceae. Flora de Guerrero, Fascículo 34. Facultad de Ciencias, Universidad Nacional Autónoma de México, México D.F.

Forero E. 1983. Connaraceae. Flora de Veracruz, Fascículo 28. Instituto de Ecología A.C., Xalapa.

Forero E. 2007. Una nueva especie mexicana de Rourea (Connaraceae). Caldasia 29:19-21.

Forero E. and González F. 2001. Connaraceae. Flora de Nicaragua. Monographs on Systematic Botany from the Missouri Botanical Garden, Vol. 85, pp. 625-653. Missouri Botanical Garden Press, St. Luis.

Fryxell P. and Guadarrama M.A. 2001. New Mexican species of Byttneria (Sterculiaceae), Bakeridesia (Malvaceae), and Triumfetta (Tiliaceae). Brittonia 53:59-65.

Fryxell P.A. 1990. Malvaceae. Flora of Chiapas, Part 3. California Academy of Sciences, San Frascisco..

Fryxell P.A. 1992. Malvaceae. Flora de Veracruz, Fascículo 68. Instituto de Ecología A.C., Xalapa.

Fryxell P.A. 2001. Malvaceae. Flora de Nicaragua. Monographs on Systematic Botany from the Missouri Botanical Garden, Vol. 85, pp. 1293-1322. Missouri Botanical Garden Press, St. Luis.

Galindo B.G. and Fernández R. 2002. Inventario florístico del municipio de Amacuzac, Morelos, México. Polibotánica 13:107-135.

Gallardo C. 1996. Parque Ecológico La Vainilla. Estudios florísticos en Guerrero, Fascículo 8. Facultad de Ciencias, Universidad Nacional Autónoma de México, México D.F.

Gallardo-Hernández C. 2004. Dilleniaceae. Flora de Veracruz, Fascículo 134. Instituto de Ecología A.C., Xalapa.

Gentry A.H. 1982. Bignoniaceae. Flora de Veracruz, Fascículo 24. Instituto de Ecología A.C., Xalapa. 
Appendix 1. Continuation.

Gentry A.H. 2001a. Apocynaceae. Flora de Nicaragua. Monographs on Systematic Botany from the Missouri Botanical Garden, Vol. 85, pp. 116-132. Missouri Botanical Garden Press, St. Luis.

Gentry A.H. 2001b. Bignoniaceae. Flora de Nicaragua. Monographs on Systematic Botany from the Missouri Botanical Garden, Vol. 85, pp. 403-428. Missouri Botanical Garden Press, St. Luis.

Gentry A.H. 2001c. Passifloraceae. Flora de Nicaragua. Monographs on Systematic Botany from the Missouri Botanical Garden, Vol. 85, pp.1913-1922. Missouri Botanical Garden Press, St. Luis.

Gereau R.E. 2001. Basellaceae. Flora de Nicaragua. Monographs on Systematic Botany from the Missouri Botanical Garden, Vol. 85, pp. 395-396. Missouri Botanical Garden Press, St. Luis.

Gillespie L.J. 1993. A synopsis of Neotropical Plukenetia (Euphorbiaceae) including two new species. Systematic Botany 18:575-592.

González C.O., Giménez de Azcárate J., García J.P. and Aguirre J.R.R. 2007. Flórula vascular de la sierra de Catorce y territorios adyacentes, San Luis Potosí, México. Acta Botanica Mexicana 78:1-38.

González-Elizondo M.S., González-Elizondo M. and Cortés-Ortíz A. 1993. Vegetación de la Reserva de la Biosfera "La Michilia", Durango, México. Acta Botanica Mexicana 22:1-104.

Grether R., Martínez-Bernal A., Luckow M. and Zárate S. 2006. Mimosaceae tribu Mimoseae. Flora del Valle de Tehuacán-Cuicatlán, Fascículo 44. Instituto de Biología, Universidad Nacional Autónoma de México, México.

Guevara S., Meave J., Moreno-Casasola P., Laborde J. and Castillo S. 1994. Vegetación y flora de potreros en la Sierra de Los Tuxtlas. Acta Botanica Mexicana 28:1-27.

Hahn W.J. 2001. Hydrangeaceae. Flora de Nicaragua. Monographs on Systematic Botany from the Missouri Botanical Garden, Vol. 85, pp. 1151. Missouri Botanical Garden Press, St. Luis.

Hamilton C.W. and Pool A. 2001. Vitaceae. Flora de Nicaragua. Monographs on Systematic Botany from the Missouri Botanical Garden, Vol. 85, 2536-2543. Missouri Botanical Garden Press, St. Luis.

Hartman R.L. and Stuessy T.F. 1983. Revision of Otopappus (Compositae, Heliantheae). Systematic Botany 8:185-210.

Hauk W.D. 1997. A review of the genus Cydista (Bignoniaceae). Annals of the Missouri Botanical Garden 84:815-840.

Hauk W.D. 1998. A review of the genus Paragonia (Bignoniaceae). Annals of the Missouri Botanical Garden 85:460-474.

Holm R.W. 1950. The American species of Sarcostemma R. Br. (Asclepiadaceae). Annals of the Missouri Botanical Garden 37:447-560.

Holmes W.C. 1990. The genus Mikania (Compositae-Eupatorieae) in Mexico. Sida 5:1-45.

Hou D. 1955.A revision of a genus Celastrus. Annals of the Missouri Botanical Garden 43:215-302.

Howard R.A. 2001. Polygonaceae. Flora de Nicaragua. Monographs on Systematic Botany from the Missouri Botanical Garden, Vol. 85, 2167-2176. Missouri Botanical Garden Press, St. Luis.

Huft M.J. 1994. Smilacaceae. In Davidse G., Sousa S., Knapp M. and Chiang F. Eds. Flora Mesoamericana Vol. 6, pp. 20-25. Instituto de Biología, Universidad Nacional Autónoma de México, Missouri Botanical Garden and The Natural History Museum (London), México D.F.

Huft M.J. 2001 a. Loganiaceae. Flora de Nicaragua. Monographs on Systematic Botany from the Missouri Botanical Garden, Vol. 85, pp. 1235-1239. Missouri Botanical Garden Press, St. Luis.

Huft M.J. 2001b. Smilacaceae. Flora de Nicaragua. Monographs on Systematic Botany from the Missouri Botanical Garden, Vol. 85, pp. 2372-2376. Missouri Botanical Garden Press, St. Luis.

Iltis H.H. 2001. Capparaceae. Flora de Nicaragua. Monographs on Systematic Botany from the Missouri Botanical Garden, Vol. 85, pp. 566-584. Missouri Botanical Garden Press, St. Luis.

Jeffrey C. 2001. Cucurbitaceae. Flora de Nicaragua. Monographs on Systematic Botany from the Missouri Botanical Garden, Vol. 85, pp. 688-717. Missouri Botanical Garden Press, St. Luis.

Jiménez J., Vega K. and Cruz R. 2011. Balsas (Sapindaceae), género nuevo de la Cuenca del Río Balsas en el estado de Guerrero, México. Novon: a journal for botanical nomenclature 21:196-200.

Johnson D.M. 1986. Revision of the Neotropical genus Callaeum (Malpighiaceae). Systematic Botany 11:335-353.

Johnston M.C. 2001. Rhamnaceae. Flora de Nicaragua. Monographs on Systematic Botany from the Missouri Botanical Garden, Vol. 85, pp. 2192-2200. Missouri Botanical Garden Press, St. Luis.

Jørgensen P.M. and León-Yánez S. Eds. 1999. Catalogue of the vascular plants of Ecuador. Monographs on Systematic Botany from the Missouri Botanical Garden, Vol. 75. Missouri Botanical Garden Press, St. Luis.

Juárez-Jaimes V. and Stevens W.D. 1995. Una nueva especie Marsdenia (Asclepiadaceae) de México. Novon: a journal for botanical nomenclature 5:337-339.

Juárez-Jaimes V. and Campos-Villanueva A. 2003. Especie nueva de Marsdenia (Asclepiadaceae), de Los Tuxtlas, Veracruz, México. Anales del Instituto de Biología de la Universidad Nacional Autónoma de México. Serie Botánica 74:73-78.

Juárez-Jaimes V. and Stevens W.D. 2005.Una nueva especie de México y Centroamérica, Marsdenia hiriartiana (Apocynaceae, Asclepiadoideae, Marsdenieae). Novon 15:552-554. 
Appendix 1. Continuation.

Juárez-Jaimes V., Stevens W.D. and Lozada L.P. 2009. Gonolobus spiranthus (Apocynaceae, Asclepiadoideae), una nueva especie de la vertiente del Pacífico Mexicano. Novon: a journal for botanical nomenclature 19:479-481.

Juárez-Jaimes V. and Ángeles-Trujillo A.L.M. 2013. El género Marsdenia (Apocynaceae: Asclepiadoideae) en Guerrero. Revista Mexicana de Biodiversidad 84:425-438.

Knapp S. 2010. Four new vining species of Solanum (Dulcamaroid Clade) from montane habitats in tropical America. PLoS ONE 5:e10502.

Knapp S. 2013. A revision of the Dulcamaroid Clade of Solanum L. (Solanaceae). PhytoKeys 22:1-432.

León de la Luz J.L., Coria R.C. and Cansino J. 1995. Listados Florísticos de México XI. Reserva de la Biosfera El Vizcaíno, Baja California Sur. Instituto de Biología, Universidad Nacional Autónoma de México, México D.F.

León de la Luz J.L., Domínguez-Cadena R. and Medel-Narváez A. 2012. Florística de la selva baja caducifolia de la Península de Baja California. Botanical Sciences 90:143-162.

Levy S.I., Aguirre J.R., García J.D. and Martínez M.M.R. 2006. Aspectos florísticos de Lacanhá Chansayab, Selva Lacandona, Chiapas. Acta Botanica Mexicana 77:69-98.

Liede S. and Meve U. 2004.Revision of Metastelma (Apocynaceae-Asclepiadoideae) in southwestern North America and Central America. Annals of the Missouri Botanical Garden 91:31-86.

Lira R. 2001. Cucurbitaceae. Flora del Bajío y de regiones adyacentes, Fascículo 92. Instituto de Ecología A.C., Pátzcuaro.

Lira R., Rodríguez-Jiménez C., Alvarado J.L., Rodríguez I., Castrejón J. and Domínguez-Mariani A. 1998. Diversidad and importancia de la familia Cucurbitaceae en México. Acta Botanica Mexicana 42:43-77.

Lira R. and Rodríguez I. 1999. Cucurbitaceae. Flora del Valle de Tehuacán-Cuicatlán, Fascículo 22. Instituto de Biología, Universidad Nacional Autónoma de México, México D.F.

Lira R. and Monro A.K. 2009.Cucurbitaceae. In Davidse G., Sousa S., Knapp M. and Chiang F. Eds. Flora Mesoamericana Vol. 4, Parte 1, pp. 1-49. Instituto de Biología, Universidad Nacional Autónoma de México, Missouri Botanical Garden and The Natural History Museum (London), México D.F.

Lira-Noriega A., Guevara S., Laborde J. and Sánchez-Ríos G. 2007. Composición florística en potreros de Los Tuxtlas, Veracruz, México. Acta Botanica Mexicana 80:59-87.

Lleras E. 2001. Trigoniaceae. Flora de Nicaragua. Monographs on Systematic Botany from the Missouri Botanical Garden, Vol. 85, pp. 2468-2469. Missouri Botanical Garden Press, St. Luis.

Lombardi J.A. 2000. Vitaceae- Géneros Ampelocissus, Ampelopsis y Cissus. Flora Neotropica 80:1-251.

Lombardi J.A. 2005. Three new species of Vitaceae from Mesoamerica. Novon: a journal for botanical nomenclature 15:562-567.

López-Pérez Y., Tejero-Díez J.D., Torres-Díaz A.N. and Luna-Vega I. 2011. Flora del bosque mesófilo de montaña y vegetación adyacente en Avándaro, Valle de Bravo, estado de México, México. Boletín de la Sociedad Botánica de México 88:35-53.

López-Sandoval J.A., Koch S.D., Vázquez-García L.M., Munguía-Lino G. and Morales-Rosales E.J. 2010. Estudio florístico de la parte central de la barranca Nenetzingo, municipio de Ixtapan de la Sal, Estado de México. Polibotánica 30:9-33.

Lorence D.H. and Nee M. 1987. Randia retroflexa (Rubiaceae), a new species from Southern Mexico. Brittonia 39:371-375.

Lozada-Pérez L. 2000. Marsdenia gallardoae (Asclepiadaceae), una nueva especie de Chiapas y Oaxaca, México. Novon: a journal for botanical nomenclature 10:128-131.

Lozada-Pérez L. 2010. Polystemma mirandae (Apocynaceae, Asclepiadoideae), una nueva especie de México. Novon: a journal for botanical nomenclature 20:429-431.

Luna I., Ocegueda S. and Alcántara O. 1994. Florística y notas biogeográficas del bosque mesófilo de montaña del municipio de Tlanchinol, Hidalgo, México. Anales del Instituto de Biología de la Universidad Nacional Autónoma de México. Serie Botánica 65:31-62.

MacDougal J.M. 1995. Passiflora sanctae-mariae in series Luteae (Passifloraceae), a new species and series in subgenus Decaloba. Novon: a journal for botanical nomenclature 5:48-51.

Martínez E., Ramos C.H. and Chiang F. 1994. Lista florística de la Lacandona, Chiapas. Boletín de la Sociedad Botánica de México 54:99-177.

Martínez E., Sousa M. and Ramos C.H. 2001. Listados Florísticos de México XXII. Región de Calakmul, Campeche. Instituto de Biología y Facultad de Ciencias, Universidad Nacional Autónoma de México, México D.F.

Martínez E. and Ramos C.H. 2007. Un nuevo género de Anacardiaceae de la península de Yucatán. Acta Botanica Hungarica 49:353-358.

Martínez E. and Ramos C.H. 2012. Bignoniaceae. Flora del Valle de Tehuacán-Cuicatlán, Fascículo 104. Instituto de Biología, Universidad Nacional Autónoma de México, México D.F.

Martínez M., Cruz R., Castrejón J.F., Valencia S., Jiménez J. and Ruiz-Jiménez C.A. 2004. Flora vascular de la porción guerrerense de la Sierra de Taxco, Guerrero, México. Anales del Instituto de Biología de la Universidad Nacional Autónoma de México. Serie Botánica 75:105-189.

Martínez-Bernal A., Duno-de Stefano R. and Lorena-Can L. 2011. Los géneros Cajanus y Rhynchosia (Leguminosae, Papilionoideae, Phaseoleae, Cajaninae) en la península de Yucatán, México. Revista Mexicana de Biodiversidad 82:1098-1107. 
Appendix 1. Continuation.

Martínez-Camilo R., Pérez-Farrera M.A. and Martínez-Meléndez N. 2012. Listado de plantas endémicas y en riesgo de la Reserva de la Biosfera El Triunfo, Chiapas, México. Botanical Sciences 90:263-285.

Martínez-García J. 1984. Phytolaccaceae. Flora de Veracruz, Fascículo 36. Instituto de Ecología A.C., Xalapa.

Martínez-García J. and Avendaño S. 1996. Basellaceae. Flora de Veracruz, Fascículo 90. Instituto de Ecología A.C., Xalapa.

Martínez-Meléndez J., Pérez-Farrera M.A. and Farrera-Sarmiento O. 2008. Inventario florístico del Cerro El Cebú y zonas adyacentes en la Reserva de la Biosfera El Triunfo (Polígono V), Chiapas, México. Boletín de la Sociedad Botánica de México 82:21-40.

Mayorga R., Luna I. and Alcántara O. 1998. Florística del bosque mesófilo de montaña de Molocotlán, Molango-Xochicoatlán, Hidalgo, México. Boletín de la Sociedad Botánica de México 63:101-119.

McDonald A. 1993. Convolvulaceae I. Flora de Veracruz, Fascículo 73. Instituto de Ecología A.C., Xalapa.

McDonald A. 1994. Convolvulaceae II. Flora de Veracruz, Fascículo 77. Instituto de Ecología A.C., Xalapa.

McDonald J.A. 2008. Merremia cielensis (Convolvulaceae: Merremiae): a new species and narrow endemic from tropical Northeast Mexico. Systematic Botany 33:552-555.

McVaugh R. 1984. Compositae. Flora Novo-Galiciana Vol. 12. University of Michigan Press, Ann Arbor.

McVaugh R. 1987. Leguminosae. Flora Novo-Galiciana Vol. 5. University of Michigan Press, Ann Arbor.

McVaugh R. 1989. Bromeliaceae to Dioscoreaceae. Flora Novo-Galiciana Vol. 15. Contributions from University of Michigan Herbarium, Ann Arbor.

McVaugh R. 2001. Ochnaceae to Loasaceae. Flora Novo-Galiciana Vol. 3. Contributions from University of Michigan Herbarium, Ann Arbor.

McVaugh R. and Schmid R. 1967. Novelties in Satureja sect. Gardoquia (Labiatae). Brittonia 19:261-267.

Medina G.C. and Rodríguez L.S.J. 1993. Estudio florístico de la cuenca del río Chiquito de Morelia, Michoacán, México. Flora del Bajío y de regiones adyacentes, Fascículo complementario 4. Instituto de Ecología A.C., Pátzcuaro.

Medina G.C., Guevara-Féfer F., Martínez M.A., Silva-Sáenz P., Chávez-Carbajal M.A. and García I. 2000. Estudio florístico en el área de la comunidad indígena de Nuevo San Juan Parangaricutiro, Michoacán, México. Acta Botanica Mexicana 52:5-41.

Medina L.R. 2001. Basellaceae. Flora del Valle de Tehuacán-Cuicatlán, Fascículo 35. Instituto de Biología, Universidad Nacional Autónoma de México, México D.F.

Medina-Lemos R. and Fonseca R.M. 2009. Anacardiaceae. Flora del Valle de Tehuacán-Cuicatlán, Fascículo 71. Instituto de Biología, Universidad Nacional Autónoma de México, México D.F.

Mennega A.M.W. 2001. Hippocrateaceae. Flora de Nicaragua. Monographs on Systematic Botany from the Missouri Botanical Garden, Vol. 85, pp. 1142-1148. Missouri Botanical Garden Press, St. Luis.

Miller J.S. 2001. Boraginaceae. Flora de Nicaragua. Monographs on Systematic Botany from the Missouri Botanical Garden, Vol. 85, pp. 435-455. Missouri Botanical Garden Press, St. Luis.

Miller J.S. 2012. Heliotropiaceae. In Davidse G., Sousa S., Knapp M. and Chiang F. Eds. Flora Mesoamericana Vol. 4, Parte 2, pp. 310-317. Instituto de Biología, Universidad Nacional Autónoma de México, Missouri Botanical Garden and The Natural History Museum (London), México D.F.

Miller J.S. 2012. Boraginaceae. In Davidse G., Sousa S., Knapp M. and Chiang F. Eds. Flora Mesoamericana Vol. 4, Parte 2, pp. 288-291. Instituto de Biología, Universidad Nacional Autónoma de México, Missouri Botanical Garden and The Natural History Museum (London), México D.F.

Monro A.K. and Rodríguez A. 2009. Three new species and a nomenclatural synopsis of Urera (Urticaceae) from Mesoamerica. Annals of the Missouri Botanical Garden 96:268-285.

Morales J.F. 1996. Una nueva especie de Tintinnabularia (Apocynaceae). Novon: a journal for botanical nomenclature 6:392-394.

Morales J.F. 1997. A reevaluation of Echites and Prestonia sect. Coalitae (Apocynaceae). Brittonia 49:328-336.

Morales J.F. 1998. A synopsis of the genus Mandevilla (Apocynaceae) in Mexico and Central America. Brittonia 50:214-232.

Morales J.F. 2002a. Studies in Neotropical Apocynaceae I: A revision of the genus Laubertia. Rhodora 104:170-185.

Morales J.F. 2002b. Studies in Neotropical Apocynaceae II: A review of the genus Fernaldia. Rhodora 104:186-200.

Morales J.F. 2005a. Estudios en las Apocynaceae neotropicales XIX: La familia Apocynaceae s. str. (Apocynoideae, Rauvolfioideae) de Costa Rica. Darwiniana 43:90-191.

Morales J.F. 2005b. Estudios en las Apocynaceae neotropicales XV: Sinopsis del género Thoreauea (Apocynoideae, Echiteae), con una nueva especie de Veracruz, México. Brittonia 57:258-263.

Morales J.F. 2006. Estudios en las Apocynaceae neotropicales XXVIII: La familia Apocynaceae (Apocynoideae, Rauvolfioideae) de El Salvador, Centroamérica. Darwiniana 44:453-489.

Morales J.F. 2009. La familia Apocynaceae (Apocynoideae, Rauvolfioideae) en Guatemala. Darwiniana 47:140-184.

Morales J.F. and Williams J.K. 2004. Allotoonia, a new Neotropical genus of Apocynaceae based on a subgeneric segregate of Echites. Sida 21:133-158.

Morales J.F. and Williams J.K. 2005. Una nueva combinación en el género Allotoonia (Apocynaceae, Apocynoideae, Echiteae). Lankesteriana 5:119-120. 
Appendix 1. Continuation.

Moreno P.P. 2001.Celastraceae. Flora de Nicaragua. Monographs on Systematic Botany from the Missouri Botanical Garden, Vol. 85, pp. 596-600. Missouri Botanical Garden Press, St. Luis.

Moura T.M., Mansano V.F., Gereau R. and Tozzi A.M.G.A. 2013. Mucuna jarocha (Leguminosae-Papilionoideae-Phaseoleae), a new species from Mexico. Phytotaxa 89:43-46.

Nash D.L. 1979. Polemoniaceae. Flora de Veracruz, Fascículo 7. Instituto de Ecología A.C., Xalapa.

Nash D.L. and Moreno N.P. 1981. Boraginaceae. Flora de Veracruz, Fascículo 18. Instituto de Ecología A.C., Xalapa.

Nash D.L. and Nee M. 1984. Verbenaceae. Flora de Veracruz, Fascículo 41. Instituto de Ecología A.C., Xalapa.

Nee M. 1984. Ulmaceae. Flora de Veracruz, Fascículo 40. Instituto de Ecología A.C., Xalapa.

Nee M. 1986. Solanaceae I. Flora de Veracruz, Fascículo 49. Instituto de Ecología A.C., Xalapa.

Nee M. 1993a. Cucurbitaceae. Flora de Veracruz, Fascículo 74. Instituto de Ecología A.C., Xalapa.

Nee M. 1993b. Solanaceae II. Flora de Veracruz, Fascículo 72. Instituto de Ecología A.C., Xalapa.

Nee M. 1999. Flacourtiaceae. Flora de Veracruz, Fascículo 111. Instituto de Ecología A.C., Xalapa.

Nesom G.L. 1988. Studies in mexican Archibaccharis (Compositae). Phytologia 65:120-128.

Ochoterena-Booth H. and Delgado A. 1994. Contribuciones a la taxonomía de Ramirezella (Leguminosae, Papilionideae). Anales del Instituto de Biología de la Universidad Nacional Autónoma de México. Serie Botánica 65:7-19.

Olvera-Luna A.R., Gama-López S. and Delgado-Salinas A. 2012. Fabaceae Tribu Aeschynomeneae. Flora del Valle de TehuacánCuicatlán, Fascículo 107. Instituto de Biología, Universidad Nacional Autónoma de México, México D.F.

Ortega J.F. and Ortega R.V. 1998. Una nueva especie de Aristolochia (Aristolochiaceae) de Chiapas, México. Acta Botanica Mexicana 44:1-6.

Ortiz R. 2001. Menispermaceae. Flora de Nicaragua. Monographs on Systematic Botany from the Missouri Botanical Garden, Vol. 85, pp. 1432-1442. Missouri Botanical Garden Press, St. Luis.

Padilla E., Cuevas R. and Koch S.D. 2008. Plantas vasculares y vegetación de la parte alta del arroyo Agua Fría, municipio de Minatitlán, Colima, México. Acta Botanica Mexicana 84:25-72.

Palmas-Pérez S., Krömer T., Dressler S. and Arévalo-Ramírez J.A. 2013. Diversidad y distribución de Marcgraviaceae en México. Revista Mexicana de Biodiversidad 84:170-183.

Panero J.L. and Dávila P. 1998. The family Schisandraceae: a new record for the Flora of Mexico. Brittonia 50:87-90.

Pankhurst R.J. 2001. Rosaceae. Flora de Nicaragua. Monographs on Systematic Botany from the Missouri Botanical Garden, Vol. 85, pp. 2202-2206. Missouri Botanical Garden Press, St. Luis.

Pérez-García E.A., Meave J. and Gallardo C. 2001. Vegetación y flora de la región de Nizanda, Istmo de Tehuantepec, Oaxaca, México. Acta Botanica Mexicana 56:19-88.

Pérez L.A., Sousa M., Hanan A.M., Chiang F. and Tenorio P. 2005. Vegetación terrestre. In: Bueno J., Álvarez F. and Santiago S. Eds. Biodiversidad del estado de Tabasco, pp. 65-110, Instituto de Biología, UNAM-CONABIO, México D.F.

Pérez-Calix E. 2004. Hydrangeaceae. Flora del Bajío y de regiones adyacentes, Fascículo 126. Instituto de Ecología A.C., Pátzcuaro.

Pérez-Calix E. and Carranza E. 1999. Ulmaceae. Flora del Bajío y de regiones adyacentes, Fascículo 75. Instituto de Ecología A.C., Pátzcuaro.

Pérez-Farrera M.A. 2004. Flora y vegetación de la Reserva de la Biósfera El Triunfo: Diversidad, riqueza y endemismo. In: PérezFarrera M.A., Martínez-Meléndez N., Hernández-Yáñez A. and Arreola-Muñoz A.V. Eds. Reserva de la Biósfera El Triunfo. Tras una Década de Conservación, pp. 77-100, Universidad de Ciencias y Artes de Chiapas, Tuxtla Gutiérrez.

Pérez-Farrera M.A., Martínez-Camilo R., Martínez-Meléndez N., Farrera-Sarmiento O. and Maza-Villalobos S. 2012. Listado florístico del Cero Quetzal (Polígono III), de la Reserva de la Biosfera El Triunfo, Chiapas. Botanical Sciences 90:113-142.

Pfeifer H.W. 1966. Revision of the North and Central American hexandrous species of Aristolochia (Aristolochiaceae). Annals of the Missouri Botanical Garden 53:115-196.

Pool A. 2001a. Nyctaginaceae. Flora de Nicaragua. Monographs on Systematic Botany from the Missouri Botanical Garden, Vol. 85, pp. 1581-1592. Missouri Botanical Garden Press, St. Luis.

Pool A. 2001b. Ranunculaceae. Flora de Nicaragua. Monographs on Systematic Botany from the Missouri Botanical Garden, Vol. 85, pp. 2190-2192. Missouri Botanical Garden Press, St. Luis.

Pool A. 2001c. Urticaceae. Flora de Nicaragua. Monographs on Systematic Botany from the Missouri Botanical Garden, Vol. 85 , pp. 2479-2495. Missouri Botanical Garden Press, St. Luis.

Pool A. 2007a. A review of the genus Distictis (Bignoniaceae). Annals of the Missouri Botanical Garden 94:791-820.

Pool A. 2007b. A revision of the genus Pithecoctenium (Bignoniaceae). Annals of the Missouri Botanical Garden 94:622-642.

Pool A. and Rueda R. 2001. Verbenaceae. Flora de Nicaragua. Monographs on Systematic Botany from the Missouri Botanical Garden, Vol. 85, pp. 2497-2525. Missouri Botanical Garden Press, St. Luis.

Pool A., Porter D.M. and Chiang F. 2001. Rutaceae. Flora de Nicaragua. Monographs on Systematic Botany from the Missouri Botanical Garden, Vol. 85, pp. 2285-2303. Missouri Botanical Garden Press, St. Luis.

Prance G.T. 2001. Dichapetalaceae. Flora de Colombia 20:1-62. 
Appendix 1. Continuation.

Prather A. 1999. Systematics of Cobaea (Polemoniaceae). Monographs on Systematic Botany from the Missouri Botanical Garden, Vol. 57. Missouri Botanical Garden Press, St. Luis.

Puig-Magrinyà F. and Carrillo-Reyes P. 2004.Aproximación a la flora y Vegetación del Cañón del Río Chico, Monte Escobedo, Zacatecas. In: Vázquez-García J.A and Vargas-Rodríguez Y.L. Eds. Flora del Norte de Jalisco y Etnobotánica Huichola, pp. 69-76, Universidad de Guadalajara, CUCBA-CUCSH, Guadalajara.

Quero H.J. 1994. Palmae (Arecaceae). Flora de Veracruz, Fascículo 81. Instituto de Ecología A.C., Xalapa.

Read R.W., Henderson A., Ulloa C. and Evans R.J. 2001. Arecaceae. Flora de Nicaragua. Monographs on Systematic Botany from the Missouri Botanical Garden, Vol. 85, pp 192-228. Missouri Botanical Garden Press, St. Luis.

Redonda-Martínez R. and Villaseñor-Ríos J.L. 2001. Asteraceae. Tribu Senecioneae. Flora del Valle de Tehuacán-Cuicatlán, Fascículo 89. Instituto de Biología, Universidad Nacional Autónoma de México, México D.F.

Rendón-Sandoval F.J., Ramírez-Delgadillo R. and Frías-Castro A. 2011. Una especie nueva de Combretum (Combretaceae, sección Combretum) de la Costa del Occidente de México. Novon: a journal for botanical nomenclature 21:483-486.

Reyes J.A., González F. and García J.D. 1996. Flora vascular de la Sierra Monte Grande, municipio de Charcas, San Luis Potosí, México. Boletín de la Sociedad Botánica de México 58:31-42.

Reyes-García A., Sousa M. and León-Velasco M.E. 2011. Inventario florístico, sinecología y aspectos fitogeográficos de la Reserva de la Biosfera la Sepultura, Sierra Madre de Chiapas. In: Álvarez Noguera F. Coordinador(es). Chiapas. Estudios sobre su diversidad biológica, pp. 71-183, Instituto de Biología, Universidad Nacional Autónoma de México, México D.F.

Reyes-García T., Maradiaga-Ceceña F.S., Catalán-Heverástico C., Ávila-Sánchez P. and Jímenez-Hernández J. 2012. Flora leñosa del municipio de Cocula, Guerrero, México. Polibotánica 34:21-49.

Rico M.L. 2000. El género Acacia (Leguminosae, Mimosoideae) en el estado de Oaxaca, México. Anales del Jardín Botánico de Madrid 58:251-302.

Rico L., Sousa M., Grether R., Hernández H.M., Andrade G., Zárate S., Pool A., Zarucchi J.M. and Luckow M. 2001. Mimosaceae. Flora de Nicaragua. Monographs on Systematic Botany from the Missouri Botanical Garden, Vol. 85, pp. $1445-1507$. Missouri Botanical Garden Press, St. Luis.

Rico M.L. and Fonseca R.M. 2005. Acaciae (Mimosaceae). Flora de Guerrero, Fascículo 25. Facultad de Ciencias, Universidad Nacional Autónoma de México, México D.F.

Robbins R.L. 2001. Sapindaceae. Flora de Nicaragua. Monographs on Systematic Botany from the Missouri Botanical Garden, Vol. 85, pp. 2307-2332. Missouri Botanical Garden Press, St. Luis.

Robinson H. 1976. Three new Asteraceae from Guerrero, Mexico. Phytologia 33:285-292.

Romero R.S. and Rojas E.C.Z. 1991. Estudio florístico de la región de Huehuetoca, Estado de México. Acta Botanica Mexicana 14:33-57.

Romero-Romero M.A., Castillo S., Meave J. and Van der Wal H. 2000. Análisis florístico de la vegetación secundaria derivada de la selva húmeda de montaña de Santa Cruz Tepetotutla (Oaxaca, México). Boletín de la Sociedad Botánica de México 67:89-106.

Rudd V. 1991. Nissolia gentryi (Leguminosae, Papilionoideae), a new species from Sonora and Sinaloa, Mexico. Acta Botanica Mexicana 15:23-27.

Rydberg P.A. 1924. The section Tuberarium of the genus Solanum in Mexico and Central America: Concluding paper. Bulletin of the Torrey Botanical Club 51:127-165.

Rzedowski J. and Calderón de Rzedowski G. 1998. Apocynaceae. Flora del Bajío y de regiones adyacentes, Fascículo 70. Instituto de Ecología A.C., Pátzcuaro.

Rzedowski J. and Calderón de Rzedowski G. 1999. Anacardiaceae. Flora del Bajío y de regiones adyacentes, Fascículo 78. Instituto de Ecología A.C., Pátzcuaro.

Rzedowski J. and Calderón de Rzedowski G. 2000. Phytolaccaceae. Flora del Bajío y de regiones adyacentes, Fascículo 91. Instituto de Ecología A.C., Pátzcuaro

Rzedowski J. and Calderón de Rzedowski G. 2003. Valerianaceae. Flora del Bajío y de regiones adyacentes, Fascículo 112. Instituto de Ecología A.C., Pátzcuaro.

Rzedowski J. and Calderón de Rzedowski G. 2005a. Rosaceae. Flora del Bajío y de regiones adyacentes, Fascículo 135. Instituto de Ecología A.C., Pátzcuaro.

Rzedowski J. and Calderón de Rzedowski G. 2005b. Vitaceae. Flora del Bajío y de regiones adyacentes, Fascículo 131. Instituto de Ecología A.C., Pátzcuaro.

Rzedowski J., Calderón de Rzedowski G. and Villarreal J.A. 1995. Notas sobre algunas Polemoniaceae mexicanas. Acta Botanica Mexicana 31:55-61.

Rzedowski J., Calderón de Rzedowski G. and Carrillo-Reyes P. 2011. Compositae-Heliantheae II. Flora del Bajío y de regiones adyacentes, Fascículo 172. Instituto de Ecología A.C., Pátzcuaro.

Salas-Morales S.H., Schibli L., Nava-Zafra A. and Saynes-Vázquez A. 2007. Flora de la costa de Oaxaca, México (2): Lista florística comentada del Parque Nacional Huatulco. Boletín de la Sociedad Botánica de México 81:101-130. 
Appendix 1. Continuation.

Sánches-Garfias B., Ibarra-Manríquez G. and González-García L. 1991. Manual de identificación de frutos y semillas anemócoros de árboles y lianas de la Estación "Los Tuxtlas", Veracruz, México. Cuadernos del Instituto de Biología 12. Instituto de Biología, Universidad Nacional Autónoma de México y The New York Botanical Garden, México D.F.

Santana J. 2011. Ulmaceae. Flora de Guerrero, Fascículo 44. Facultad de Ciencias, Universidad Nacional Autónoma de México, México D.F.

Schubert B.G. and Téllez O. 2001. Dioscoreaceae. Flora de Nicaragua. Monographs on Systematic Botany from the Missouri Botanical Garden, Vol. 85, 807-814. Missouri Botanical Garden Press, St. Luis.

Schultz G.P. 2005. Vascular Flora of the El Edén Ecological Reserve, Quintana Roo, Mexico. Journal of the Torrey Botanical Society 132:311-322.

Short M.J. and Helgason T. 2009. Polemoniaceae. In Davidse G., Sousa S., Knapp M. and Chiang F. Eds. Flora Mesoamericana Vol. 4, Parte 1, pp. 768-772. Instituto de Biología, Universidad Nacional Autónoma de México, Missouri Botanical Garden and The Natural History Museum (London), México D.F.

Skog L.E. 2001. Gesneriaceae. Flora de Nicaragua. Monographs on Systematic Botany from the Missouri Botanical Garden, Vol. 85, pp. 1114-1128. Missouri Botanical Garden Press, St. Luis.

Sohmer S.H. 1977. A revision of Chamissoa (Amaranthaceae). Bulletin of the Torrey Botanical Club 104:111-126.

Solano E. and Ayala M.M. 2008. Polygonaceae. Flora del Valle de Tehuacán-Cuicatlán, Fascículo 63. Instituto de Biología, Universidad Nacional Autónoma de México, México D.F.

Sosa V., Schuber B.G. and Gómez-Pompa A. 1987. Dioscoreaceae. Flora de Veracruz, Fascículo 53. Instituto de Ecología A.C., Xalapa.

Spellenberg R. 2001. Nyctaginaceae. Flora del Bajío y de regiones adyacentes, Fascículo 93. Instituto de Ecología A.C., Pátzcuaro.

Stace C.A. 2001. Combretaceae. Flora de Nicaragua. Monographs on Systematic Botany from the Missouri Botanical Garden, Vol. 85, pp. 631-638. Missouri Botanical Garden Press, St. Luis.

Standley P.C. 1920. Six new species of plants from Mexico. Proceedings of the Biological Society of Washington 33:65-68.

Standley P.C. and Steyermark J.A. 1946. Flora of Guatemala. Fieldiana Botany 24:468-500.

Standley P.C. and Steyermark J.A. 1949. Flora of Guatemala. Fieldiana Botany 24:5-273.

Standley P.C. and Steyermark J.A. 1952. Flora of Guatemala. Fieldiana Botany 24:59-100.

Standley P.C. and Williams L.O. 1961. Flora of Guatemala. Fieldiana Botany 24:115-146.

Staples G.W. and Austin D.F. 2009. Revision of Neotropical Calycobolus and Porana (Convolvulaceae). Edinburgh Journal of Botany 66:133-153.

Stevens W.D. 1988. A synopsis of Matelea subg. Dictyanthus (Apocynaceae: Asclepiadoideae). Annals of the Missouri Botanical Garden 75:1533-1564.

Stevens W.D. 2000. New and interesting milkweeds (Apocynaceae, Asclepiadaceae). Novon: a journal for botanical nomenclature 10:242-256.

Stevens W.D. 2001a. Asclepiadaceae. Flora de Nicaragua. Monographs on Systematic Botany from the Missouri Botanical Garden, Vol. 85, pp. 234-270. Missouri Botanical Garden Press, St. Luis.

Stevens W.D. 2001b. Phytolaccaceae. Flora de Nicaragua. Monographs on Systematic Botany from the Missouri Botanical Garden, Vol. 85, pp. 1924-1928. Missouri Botanical Garden Press, St. Luis.

Stevens W.D. 2005. Fourteen new species of Gonolobus (Apocynaceae, Asclepiadoideae) from Mexico and Central America. Novon: a journal for botanical nomenclature 15:222-244.

Stevens W.D. and Montiel O.M. 2004. Gonolobus incerianus (Apocynaceae, Asclepiadoideae), una nueva especie de Mesoamérica. Novon: a journal for botanical nomenclature 14:350-353.

Strother J.L. 1999. Compositae-Heliantheae s.I. Flora of Chiapas, Part 5. California Academy of Sciences, San Frascisco..

Taylor C.M. 2001. Rubiaceae. Flora de Nicaragua. Monographs on Systematic Botany from the Missouri Botanical Garden, Vol. 85, pp. 2206-2284. Missouri Botanical Garden Press, St. Luis.

Téllez O. 1996. Smilacaceae. Flora del Valle de Tehuacán-Cuicatlán, Fascículo 11. Instituto de Biología, Universidad Nacional Autónoma de México, México D.F.

Téllez O. and Schubert B.G. 1991. Especies nuevas y colecciones notables de Dioscorea (Dioscoreaceae) en Mesoamérica. Annals of the Missouri Botanical Garden 78:245-248.

Téllez O., Rudd V.E., Crowder C., Sousa M., Delgado-Salinas A., Zarucchi J.L., Fantz P.R., Maxwell R.H., Guzmán-Teare M., Torres-Colín L., Neill D., Barneby R., Pool M., Lavin M., Germán M., Harder D., Antonio R., Klitgaard H., Ochoterena-Booth H., Hughes C., and Dorado O. 2001. Fabaceae. Flora de Nicaragua. Monographs on Systematic Botany from the Missouri Botanical Garden, Vol. 85, pp. 945-1076. Missouri Botanical Garden Press, St. Luis.

Todzia C.A. 2001a. Dilleniaceae. Flora de Nicaragua. Monographs on Systematic Botany from the Missouri Botanical Garden, Vol. 85, pp. 802-807. Missouri Botanical Garden Press, St. Luis.

Todzia C.A. 2001b. Ulmaceae. Flora de Nicaragua. Monographs on Systematic Botany from the Missouri Botanical Garden, Vol. 85, pp. 2474-2478. Missouri Botanical Garden Press, St. Luis. 
Appendix 1. Continuation.

Todzia C.A. 2001c. Violaceae. Flora de Nicaragua. Monographs on Systematic Botany from the Missouri Botanical Garden, Vol. 85, pp. 2526-2530. Missouri Botanical Garden Press, St. Luis.

Turner B. and Olsen J. 1988. Two new species of Verbesina sect. Platypteris (Asteraceae) from Jalisco, Mexico. Sida 13:39-43.

Turner B.L. 1988. A new species of Hidalgoa (Asteraceae, Coreopsideae) from Southern Mexico. Phytologia 65:379-381.

Utley J.F. 1984. Marcgraviaceae. Flora de Veracruz, Fascículo 38. Instituto de Ecología A.C., Xalapa.

Utley J.F. 2001. Marcgraviaceae. Flora de Nicaragua. Monographs on Systematic Botany from the Missouri Botanical Garden, Vol. 85, pp. 1335-1338. Missouri Botanical Garden Press, St. Luis.

Valiente-Banuet A. and Luna G.E. 1990. Una lista florística actualizada para la Reserva del Pedregal de San Ángel, México D.F. Acta Botanica Mexicana 9:13-30.

Van der Werff H. 2001. Lauraceae. Flora de Nicaragua. Monographs on Systematic Botany from the Missouri Botanical Garden, Vol. 85, pp. 1190-1206. Missouri Botanical Garden Press, St. Luis.

Vargas A. and Pérez A. 1996. Cerro Chilepepetl y alrededores (Cuenca del Balsas). Estudios florísticos en Guerrero, Fascículo 7. Facultad de Ciencias, Universidad Nacional Autónoma de México, México D.F.

Vázquez-García J.A., Nieves G., Cházaro M., Vargas-Rodríguez Y.L., Flores A. and Luquín H. 2004. Listado preliminar de plantas vasculares del norte de Jalisco y zonas adyacentes. In: Vázquez-García J.A and Vargas-Rodríguez Y.L. Eds. Flora del norte de Jalisco y etnobotánica Huichola, pp. 116-168, Universidad de Guadalajara, CUCBA-CUCSH, Guadalajara.

Villarreal J.A. 2000. Caprifoliaceae. Flora del Bajío y de regiones adyacentes, Fascículo 88. Instituto de Ecología A.C., Pátzcuaro. Villarreal J.A. 2002. Caprifoliaceae. Flora de Veracruz, Fascículo 126. Instituto de Ecología A.C., Xalapa.

Villarreal J.A., Carranza M.A., Estrada E. and Rodríguez A. 2006. Flora riparia de los ríos Sabinas y San Rodrigo, Coahuila, México. Acta Botanica Mexicana 75:1-20.

Villaseñor J.L. and Strother J.L. 1989. Tuxtla, a new genus for Zexmenia pittieri (Compositae: Heliantheae). Systematic Botany 14:529-540.

Vincent M.A. 1997. Schisandraceae. In Flora of North America Editorial Committee. Eds. Flora of North America, Vol. 3, pp. 62-63. New York and Oxford.

Webster G.L., Huft M.J. and Levin G.A. 2001. Euphorbiaceae. Flora de Nicaragua. Monographs on Systematic Botany from the Missouri Botanical Garden, Vol. 85, pp. 839-903. Missouri Botanical Garden Press, St. Luis.

Wendt T.L. 2001. Polygalaceae. Flora de Nicaragua. Monographs on Systematic Botany from the Missouri Botanical Garden, Vol. 85, pp. 2154-2467. Missouri Botanical Garden Press, St. Luis.

Williams J.K. 1995. A new species of Thenardia (Apocynaceae, Apocynoideae) from Chiapas, Mexico, with notes of the genus. Brittonia 47:403-407.

Williams J.K. 2002. A further evaluation of Echites sect. Yucatanense (Apocynaceae) with additional notes on the genus. Brittonia 54:310-317.

Willman D., Schmidt E., Heinrich M. and Rimpler H. 2000. Verbenaceae. Flora del Valle de Tehuacán-Cuicatlán, Fascículo 27. Instituto de Biología, Universidad Nacional Autónoma de México, México D.F.

Woodson R.E. and Schery R.W. 1980. Flora de Panama, Family 83. Leguminosae. Annals of the Missouri Botanical Garden 67:523-818.

Zamora C.P. 2003. Contribución al estudio florístico y descripción de la vegetación del municipio de Tenabo, Campeche, México. Polibotánica 15:1-40.

Zepeda C. and Velázquez E. 1999. El bosque tropical caducifolio de la vertiente sur de la sierra de Nanchititla, Estado de México: la composición y la afinidad geográfica de su flora. Acta Botanica Mexicana 46:29-55.

Zumaya S., Flores-Olvera H. and Borsch T. 2013. Two new Mexican endemic species of Iresine (Amaranthaceae). Systematic Botany 38:434-443. 
Guillermo IbarRa-ManRíQueZ ET AL.

Appendix 2. List of liana species of Mexico. For each family, the number of genera and species is shown in parentheses, separated by a slash. For each species we mentioned species name and their author, original publication, growth form, geographical distribution in Mexico (states) and a continental scale (Africa, America [Antilles, Middle America, South America, United States of America] and Asia), vegetation where species was found and its altitudinal range. Abbreviations: 1) Growth form: He (hemiepiphythe), Li (liana), Sc (scandent shrub); 2) Geographical distribution: USA (United States of America), MEX (Mexico), Ags (Aguascalientes), BC (Baja California), BCS (Baja California Sur), Cam (Campeche), Chis (Chiapas), Chih (Chihuahua), Coa (Coahuila), Col (Colima), DF (Distrito Federal), Dgo (Durango), Gto (Guanajuato), Gro (Guerrero), Hgo (Hidalgo), Jal (Jalisco), Mex (México), Mich (Michoacán), Mor (Morelos), Nay (Nayarit), NL (Nuevo León), Oax (Oaxaca), Pue (Puebla), Qro (Querétaro), QR (Quintana Roo), SLP (San Luis Potosí), Sin (Sinaloa), Son (Sonora), Tab (Tabasco), Tam (Tamaulipas), Tla (Tlaxcala), Ver (Veracruz), Yuc (Yucatán), Zac (Zacatecas), ANT (Antilles), MA (Middle America), Bel (Belize), CR (Costa Rica), ES (El Salvador), Gua (Guatemala), Hon (Honduras), Nic (Nicaragua), Pan (Panama), SA (South America), AF (Africa), AS (Asia); 3) Vegetation types: CF (conipherous forest), HMF (humid mountain forest), MG (mangrove), PQF (Pinus-Quercus forest), QF (Quercus forest), RF (riparian forest), SAV (Savanna), SV (Secondary vegetation), TDF (tropical dry forest), TEF (tropical evergreen forest), TF (thorn forest), TSEF (tropical semi evergreen forest), XS (xerophytic scrubland); 4) NA.: without available data.

\section{Acanthaceae (1/2)}

Mendoncia guatemalensis Standl. \& Steyerm. Publ. Field Mus. Nat. Hist. Bot. Ser. 23(5): 245. 1947. Li. MEX (Chis, Oax, Ver), MA (Gua, Hon). TEF. 60-1,100 m.

M. retusa Turrill. Bull. Misc. Inform. Kew 1919(10): 423. 1919. Li. MEX (Chis, Ver), MA (Bel, CR, Gua, Hon, Nic, Pan), SA. TDF, TEF, TSEF. 0-1,010 m.

\section{Amaranthaceae (4/6)}

Chamissoa altissima (Jacq.) Kunth var. altissima. Nov. Gen. Sp. (quarto ed.) 2(7): 197, t. 125. 1817[1818]. Li, Sc. MEX (Cam, Chis, Col, Hgo, Jal, Mich, Nay, Oax, Pue, Qro, Sin, SLP, Tab, Tam, Ver, Yuc), MA (Bel, CR, ES, Gua, Hon, Nic, Pan), ANT, SA. HMF, SV, TDF, TEF, TSEF. 160-1,120 m.

Hebanthe grandiflora (Hook.) Borsch \& Pedersen. Sendtnera 4: 20. 1997. Li. MEX (Chis, Oax), MA (Bel, CR, Hon, Nic), SA. TEF. 280-300 m.

Iresine nigra Uline \& W.L. Bray. Bot. Gaz. 21(5): 350. 1896. Sc. MEX (Cam, Chis, Gro, Nay, Oax, Ver), MA (Bel, CR, ES, Gua, Hon, Nic, Pan). PQF, SV, TDF, TSEF. 95-1,180 m.

I. palmeri (S. Watson) Standl. J. Wash. Acad. Sci. 5(11): 395. 1915. Li. USA, MEX (Hgo, NL, SLP, Tam, Ver). PQF, TEF. 20-2,200 m.

I. rzedowskii Zumaya, Flores Olv. \& Borsch. Syst. Bot. 38(2): 435-438, f. 1-2. 3a, 4. 2013. Li. MEX (Chih, Dgo, Gto, Gro, Jal, Mich, Nay, Oax, Pue, Qro, Sin). PQF, TDF, SV, XS. 1,145-2,500 m.

Pleuropetalum pleiogynum (Kuntze) Standl. J. Wash. Acad. Sci. 13(16): 369. 1923. Sc. MEX (Oax), MA (CR, Nic, Pan), SA. PQF. NA.

Anacardiaceae (3/3)

Attilaea abalak E. Martínez \& Ramos. Acta Bot. Hung. 49(3-4): 354-356, f. 1. 2007. Li, Sc. MEX (Cam, QR), MA (Gua). SV, TDF, TEF, TSEF. 40-276 m.

Rhus terebinthifolia Schltdl. \& Cham. Linnaea 5: 600-601. 1830. Sc. MEX (Chis, Dgo, Gro, Hgo, Jal, Mex, Mich, Mor, Nay, Oax, Pue, Qro, SLP, Sin, Son, Tam, Ver, Zac), MA (CR, ES, Gua, Hon). HMF, QF, TSEF, XS. 750-1,900 m.

Toxicodendron radicans (L.) Kuntze. Revis. Gen. PI. 1: 153. 1891. Li, Sc. USA, MEX (Ags, BC, BCS, Chis, Chih, Coa, Col, DF, Dgo, Gto, Gro, Hgo, Jal, Mex, Mich, Mor, Nay, NL, Oax, Pue, Qro, SLP, Sin, Son, Tam, Ver, Zac), MA (Gua), AS. BC, HMF, QF, RF. 300-2,700 m.

\section{Apocynaceae (32/158)}

Cynanchum ligulatum (Benth.) Woodson. Ann. Missouri Bot. Gard. 28(2): 210. 1941. Li. USA, MEX (Ags, Chih, Gto, Gro, Jal, Mex, Mich, Mor, Nay, Oax, Sin, Son, Zac). QF, SV, TDF, XS. 7-2,020 m.

Dictyanthus aeneus Woodson. Amer. J. Bot. 22(7): 691-692, t. 1, f. 4a. 1935. Li. MEX (Cam, Yuc). TDF, XS. 0-10 m.

D. altatensis (Brandegee) W.D. Stevens. Novon 10(3): 243. 2000. Li. MEX (Jal, Sin, Son). SV, TDF, XS. 0-750 m.

D. asper (Mill.) W.D. Stevens. Novon 9(4): 564. 1999. Li. MEX (Chis, Col, Jal, Mex, Mich, Oax, Ver), MA (ES, Gua, Hon, Nic). SV, TDF. $300 \mathrm{~m}$.

D. eximius (W.D. Stevens) W.D. Stevens. Novon 10(3): 243. 2000. Li. MEX (Chis). CF, TDF. 700-900 m.

D. hamatus (W.D. Stevens) W.D. Stevens. Novon 10(3): 243. 2000. Li. MEX (Gro, Oax). SV, TDF, TSEF. 80-200 m.

D. lautus (W.D. Stevens) W.D. Stevens. Novon 10(3): 243. 2000. Li. MEX (Col). TDF. 200-300 m.

D. parviflorus Hemsl. Biol. Cent.-Amer. Bot. 2(11): 329. 1882. Li. MEX (Chis, Gro, Mex, Mich, Mor, Oax, Pue, Ver), MA (ES, Gua). PQF, TDF. 800-2,600 m.

D. pavonii Decne. Prodr. 8: 605. 1844. Li. MEX (Chis, Col, Gto, Gro, Jal, Mex, Mich, Mor, Nay, Oax, Sin). PQF, QF, SV, TDF. $600-2,500 \mathrm{~m}$. 
D. sepicola (W.D. Stevens) W.D. Stevens. Novon 10(3): 243. 2000. Li. MEX (Jal, Nay, Sin, Son). TDF. 20-30 m.

D. suffruticosus (W.D. Stevens) W.D. Stevens. Novon 10(3): 243. 2000. Li. MEX (Oax). TDF, XS. 50-100 m.

D. tigrinus Conz. \& Standl. Contr. U.S. Natl. Herb. 23(4): 1183-1184. 1924. Li. MEX (Chis, Oax, Ver). QF, TEF. 60-1,220 m.

D. tuberosus B.L. Rob. Proc. Amer. Acad. Arts 27: 180-181. 1893. Li. MEX (Jal, Son, Zac). PQF, SV, TDF. 500-1,600 m.

D. yucatanensis Standl. Publ. Field Mus. Nat. Hist. Bot. Ser. 8(1): 37. 1930. Li. MEX (Cam, QR, Yuc). SV, TDF, TSEF. 0-200 m.

Echites turbinatus Woodson. Ann. Missouri Bot. Gard. 21(4): 615-616. 1934. Li. MEX (Chis), MA (CR, Hon, Pan). HMF, SV. 1,500$1,800 \mathrm{~m}$.

E. tuxtlensis Standl. Contr. U.S. Natl. Herb. 23: 1164. 1924. Li, Sc. MEX (Cam, Chis, Oax, Qro, QR, SLP, Tab, Ver, Yuc), MA (Bel, CR, ES, Gua, Hon). QF, SV, TDF, TEF. 110-1,100 m.

E. umbellatus Jacq. Enum. Syst. PI. 13: 1790. Li. USA, MEX (Cam, QR, Tab, Ver, Yuc), ANT, MA (Bel, Gua, Hon), SA. SV, TDF, TSEF. 0-200 m.

E. woodsonianus Monach. Bull. Torrey Bot. Club. 86: 245, f. 1. 1959. Li. MEX (Gro, Jal, Mich, Oax, QR), MA (CR, Gua, Hon, Nic). RF, SV, TDF, TSEF. 170-450 m.

E. yucatanensis Millsp. ex Standl. Publ. Field Columbian Mus. Bot. Ser. 8(1): 35. 1930. Li. MEX (Cam, Col, Gro, Jal, Mich, QR, Yuc), MA (Bel, ES, Gua, Hon, Nic). TDF, TEF, TSEF. 0-1,200 m.

Fernaldia asperoglottis Woodson. Ann. Missouri Bot. Gard. 26(2): 96-97. 1939. Li. MEX (Gro, Mex, Mich). SV, TDF. 200-1,350 m.

F. pandurata (A. DC.) Woodson. Ann. Missouri Bot. Gard. 19: 48. 1932. Li. MEX (Cam, Chis, Col, Gro, Hgo, Jal, Nay, Oax, Pue, Qro, QR, SLP, Tam, Ver, Yuc), MA (CR, ES, Gua, Hon, Nic). HMF, MG, QF, RF, SV, TDF, TSEF. 10-900 m.

Fischeria scandens DC. Cat. Pl. Horti Monsp. 112-113. 1813. Li. MEX (Chis, Oax, Pue, Tab, Ver), ANT, MA (Bel, Gua, Hon, Nic, Pan), SA. TEF. 0-1,350 m.

Forsteronia acouci (Aubl.) A. DC. Prodr. 8: 439. 1844. Li. MEX (Chis, Oax, Ver), MA (Bel, CR, Gua, Pan), SA. SV, TEF. 100-400 m.

F. myriantha Donn. Sm. Bot. Gaz. 27 (6): 435. 1899. Li. MEX (Chis, Gro, Oax, Pue, Ver), MA (Bel, CR, ES, Gua, Hon, Nic, Pan), SA. RF, SV, TDF, TEF. 0-1,250 m.

F. spicata (Jacq.) G. Mey. Prim. Fl. Esseq. 135. 1818. Li, Sc. MEX (Chis, Gro, Jal, Nay, Oax, Ver), ANT, MA (CR, ES, Gua, Hon, Nic, Pan), SA. RF, SV, TDF, TSEF. 250-275 m.

Funastrum elegans (Decne.) Schltr. Repert. Spec. Nov. Regni Veg. 13: 284. 1914. Li. MEX (Col, DF, Gro, Hgo, Jal, Mex, Mich, Oax, Pue, Qro, SLP, Tla, Ver). QF, SV, TDF. 1,500-2,400 m.

F. pannosum Schltr. Repert. Spec. Nov. Regni Veg. 13(363/367): 286. 1914. Li. MEX (BC, BCS, Chis, Chih, Dgo, Gto, Gro, Jal, Mex, Mich, Mor, Nay, Oax, Pue, Qro, SLP, Sin, Son, Tam). TDF, XS. 150-1,800 m.

Gonolobus barbatus Kunth. Nov. Gen. Sp. (quarto ed.) 3: 209, t. 239. 1818[1819]. Li. MEX (Cam, Chis, Jal, Mor, Oax, QR, Tab, Ver, Yuc), MA (CR, ES, Gua, Hon, Nic). RF, SV, TDF, TEF, TSEF. 0-1,000 m.

G. breedlovei L.O. Williams. Fieldiana, Bot. 32(4): 44-45. 1968. Li. MEX (Chis). PQF, TEF. 1,000-1,400 m.

G. chiapensis (Brandegee) Woodson. Ann. Missouri Bot. Gard. 28: 242. 1941. Li. MEX (Chis), MA (Gua). HMF, TEF. 1,325$1,800 \mathrm{~m}$.

G. chloranthus Schltdl. Linnaea 8(5): 520-521. 1834. Li. MEX (Gro, Hgo, Jal, Mich, Pue, Qro, SLP, Ver). HMF, QF, SV. 1,000$2,170 \mathrm{~m}$.

G. cteniophorus (S.F. Blake) Woodson. Ann. Missouri Bot. Gard. 28: 243. 1941. Li. MEX (Cam, Chis, QR, Tab, Ver, Yuc), MA (Bel, Gua, Hon). TDF, TEF, TSEF. 0-350 m.

G. cuajayote W.D. Stevens. Novon 15(1): 228-230, f. 5. 2005. Li. MEX (Chis), MA (ES, Gua, Nic). HMF, TEF. $150-900$ m.

G. erianthus Decne. Prodr. 8: 592. 1844. Li. MEX (Chis, Gto, Gro, Hgo, Jal, Mex, Mich, Mor, Nay, NL, Oax, Pue, SLP, Tam, Ver), MA (Gua, Hon, Nic). HMF, TDF. 300-1,500 m.

G. fraternus Schltdl. Linnaea 8(5): 521. 1833[1834]. Li. MEX (Cam, Chis, Gro, Hgo, Mich, Oax, Pue, Qro, SLP, Tab, Tam, Ver), MA (Bel, Gua). PQF, TDF, TEF. 30-1,900 m.

G. grandiflorus (Cav.) R. Br. ex Schult. Syst. Veg. 6: 61. 1820. Li. MEX (DF, Gto, Gro, Hgo, Jal, Mex, Mich, Mor, Oax, Pue, Qro, Ver, Zac). CF, RF, TDF, XS. 1,800-2,000 m.

G. incerianus W.D. Stevens \& Montiel. Novon 14(3): 350-353. 2004. Li. MEX (Chis, Oax, Ver), MA (ES, Gua, Hon, Nic). HMF, PQF. 1,300-2,700 m.

G. leianthus Donn. Sm. Bot. Gaz. 48: 296. 1909. Li. MEX (Cam, Chis, Tab), MA (Bel, Gua, Hon, Nic). HMF, SV, TEF. 40-1,250 m.

G. nemorosus Decne. Prodr. 8: 596. 1844. Li. MEX (Oax, Pue). QF, TDF, XS. 960-1,800 m.

G. niger (Cav.) R. Br. ex Schult. Syst. Veg. 6: 61. 1820. Li. MEX (Chis, Hgo, Oax, Qro, SLP, Tab, Tam, Ver), MA (Gua). RF, HMF, PQF, TEF. 0-1,700 m.

G. pectinatus Brandegee. Univ. Calif. Publ. Bot. 3(8): 387. 1909. Li. MEX (Gro, Mex, Mich, Mor, Oax, Pue). RF, TDF. 700$1,100 \mathrm{~m}$.

G. spiranthus Juárez-Jaimes, W.D. Stevens \& Lozada-Pérez. Novon 19(4): 479-481, f. 1. 2009. Li. MEX (Gro, Jal, Mex, Mich, Oax). SV, TSEF. 220-1,500 m. 
Appendix 2. Continuation.

G. stenanthus (Standl.) Woodson. Ann. Missouri Bot. Gard. 28: 243. 1941. Li. MEX (Cam, QR, Tab, Yuc), MA (Bel, Hon). TDF, TSEF. 0-200 m.

G. stenosepalus (Donn. Sm.) Woodson. Ann. Missouri Bot. Gard. 28: 243. 1941. Li. MEX (Chis), MA (Gua, Hon). CF, HMF. 1,8002,700 m.

G. uniflorus Kunth. Nov. Gen. Sp. (quarto ed.) 3: 207, t. 238. 1818[1819]. Li. MEX (Chis, Col, DF, Mex, Mich, Mor, Oax, Pue), MA (Bel, ES, Gua, Hon). HMF, QF, XS. 1,300-2,600 m.

Laubertia contorta (M. Martens \& Galeotti) Woodson. N. Amer. Fl. 29(2): 187-188. 1938. Li. MEX (Chis, Col, Gro, Jal, Mex, Mich, Mor, Nay, Oax, Pue, SLP, Sin). SV, TDF, TEF, TSEF. 50-2,050 m.

Macroscepis diademata (Ker Gawl.) W.D. Stevens. Phytologia 53(6): 405. 1983. Li. MEX (Cam, Chis, Oax, QR, Ver, Yuc), MA (Gua). CF, RF, SV, TDF, TEF, TSEF. 10-1,000 m.

M. pleistantha Donn. Sm. Bot. Gaz. 20(12): 543-544. 1895. Li. MEX (Chis), MA (CR, ES, Gua, Hon, Nic). HMF, TDF, TEF, TSEF. 600-1,000 m.

Mandevilla acutiloba (A. DC.) Woodson. Ann. Missouri Bot. Gard. 19(1): 54. 1932. Li. MEX (Chis, Gro, Oax), MA (ES, Gua, Hon). PQF, TDF. 1,000-2,240 m.

M. andrieuxii (Müll. Arg.) Hemsl. Biol. Cent.-Amer. Bot. 2(10): 316. 1881. Li. MEX (Col, Jal, Mich, Oax). TDF, TSEF. 0-900 m.

M. convolvulacea (A. DC.) Hemsl. Biol. Cent.-Amer. Bot. 2(10): 316. 1881. Li. MEX (Oax, Pue). QF, TDF, XS. 1,400-1,950 m.

M. hirsuta (Rich.) K. Schum. Nat. Pflanzenfam. 4(2): 171. 1895. Li. MEX (Chis, Oax, Tab), ANT, MA (Bel, CR, Gua, Hon, Nic, Pan), SA. SAV, SV, TEF. 30-250 m.

M. holosericea (Sessé \& Moc.) J.K. Williams. Sida 18(1): 237. 1998. Li. MEX (Ags, Chis, Gto, Gro, Hgo, Jal, Mex, Mich, Mor, Nay, Oax, Pue, Qro, Sin). QF, SV, TDF, XS. 0-2,300 m.

M. karwinskii (Müll. Arg.) Hemsl. Biol. Centr. Amer. Bot. 2: 316. 1881. Sc. MEX (Coa, Gto, Gro, Hgo, Mex, NL, Qro, SLP, Tam, Ver). HMF, QF, TDF, XS. 250-2,200 m.

M. oaxacana (A. DC) Hemsl. Biol. Cent.-Amer. Bot. 2(10): 316. 1881. Li. MEX (Oax). PQF, QF, SV. 1,650-1,880 m.

M. subsagittata (Ruiz \& Pav.) Woodson. Ann. Missouri Bot. Gard. 19(1): 69-70. 1932. Li. MEX (Cam, Chis, Gro, Hgo, Jal, Mex, Mich, Mor, Nay, Oax, Pue, Qro, QR, SLP, Sin, Tab, Tam, Ver, Yuc), ANT, MA (Bel, CR, ES, Gua, Hon, Nic, Pan), SA. PQF, QF, RF, SV TDF, TSEF. 10-1,200 m.

M. subsessilis (A. DC.) Woodson. Ann. Missouri Bot. Gard. 19(1): 59. 1932. Li. MEX (Chis, Gro, Jal, Mich, Oax, SLP), MA (ES, Gua). RF, SV, TDF, XS. 700-1,600 m.

M. torosa (Jacq.) Woodson. Ann. Missouri Bot. Gard. 19(1): 64-65. 1932. Li. MEX (Cam, Chis, Hgo, NL, QR, Qro, SLP, Tam, Ver, Yuc), ANT. QF, SV, TDF, TSEF. 2-1,950 m.

M. tubiflora (M. Martens \& Galeotti) Woodson. Ann. Missouri Bot. Gard. 19(1): 52. 1932. Li. MEX (Chis, Gro, Mich, Oax, Tab, Ver, Yuc), MA (ES, Gua, Hon, Nic). CF, HMF, SV, TDF, TSEF, XS. 400-1,700 m.

M. villosa (Miers) Woodson. Ann. Missouri Bot. Gard. 19(1): 70. 1932. Li. MEX (Chis), MA (CR, ES, Gua, Hon, Nic, Pan), SA. SAV, TDF. 640-800 m.

Marsdenia astephanoides (A. Gray) Woodson. Ann. Missouri Bot. Gard. 28: 244. 1944. Li. MEX (Col, Gro, Jal, Mich, Nay, Oax, Sin). SV, TDF. 50-1,100 m.

M. bourgaeana (Baill.) W. Rothe. Bot. Jahrb. Syst. 53: 408. 1915. Li. MEX (Chis, Mor, Oax, Ver), MA (Gua, Hon), SA, AS. HMF, TDF. 820-1,480 m.

M. callosa Juárez-Jaimes \& W.D. Stevens. Novon 7: 253-255. 1997. Li. MEX (Gro, Jal, Mich, Oax). SV, TDF, TSEF, XS. 0-1,100 m.

M. coulteri Hemsl. Biol. Cent.-Amer. Bot.) 2(11): 336-337. 1882. Li. MEX (Cam, Chis, Coa, Gto, Hgo, Jal, NL, Oax, Pue, QR, Sin, Son, Ver, Yuc). TDF, TSEF, XS. 1,000-1,600 m.

M. cuixmalensis Juárez-Jaimes \& L.O. Alvarado-Cárdenas. Candollea 65(1): 64. 2010. Li. MEX (Jal). TDF. 5-300 m.

M. edulis S. Watson. Proc. Amer. Acad. Arts 24: 61. 1889. Li. MEX (Chih, Sin, Son). TDF. 50-835 m.

M. gallardoae Lozada. Novon 10(2): 128-131, f. 1. 2000. Li. MEX (Chis, Oax). RF, TDF. 0-800 m.

M. gualanensis Donn. Sm. Bot. Gaz. 49: 456-457. 1910. Li. MEX (Chis, Jal, Oax, Yuc), MA (CR, ES, Gua, Hon, Nic). SV, TDF. 0-1,080 m.

M. hiriartiana Juárez-Jaimes \& W.D. Stevens. Novon 15(4): 552-554, f. 1. 2005. Li. MEX (Chis, QR), MA (Gua, Hon). TEF. 5$620 \mathrm{~m}$.

M. lanata (Paul G. Wilson) W.D. Stevens. SouthW. Naturalist 21(3): 406. 1976. Li. MEX (Chis, Col, Gro, Jal, Mex, Mich, Mor, Nay, Oax, Pue, Sin, Ver), MA (ES, Gua, Hon). TDF, TSEF. 10-1,500 m.

M. laxiflora Donn. Sm. Bot. Gaz. 40(1): 7-8. 1905. Li. MEX (Chis, Oax, Tab, Ver), MA (Bel, ES, Gua, Hon). HMF, TEF. 0-1,200 m.

M. macrophylla (Humb. \& Bonpl. ex Schult.) E. Fourn. Fl. Bras. 6(4): 321. 1885. Li. MEX (Chis, Mich, QR, Tab, Tam, Ver, Yuc), ANT, MA (CR, Gua, Hon, Nic, Pan), SA. HMF, SV, TEF, TSEF. 0-3 m.

M. mayana Lundell. Contr. Univ. Michigan Herb. 4: 23-24. 1940. Li. MEX (Chis, Gro, Jal, Nay, QR, Yuc), MA (Bel, Gua, Hon). RF, SV, TDF. 30-1,400 m. 
Appendix 2. Continuation.

M. mexicana Decne. Prodr. 8: 617. 1844. Li. MEX (Chis, Gro, Jal, Mex, Mich, Mor, Oax, Pue, Ver), MA (Gua). CF, PQF, SV, TDF. 100-2,200 m.

M. parvifolia Brandegee. Zoë 5: 235-236. 1908. Li. MEX (Pue). TDF, XS. 1550-1,700 m.

M. popoluca Juárez-Jaimes \& A. Campos V. Anales Inst. Biol. Univ. Nac. Autón. México, Bot. 74(1): 74-78, f. 1-2. 2003. Li. MEX (Tab, Ver). TEF. 20-200 m.

M. pringlei S. Watson. Proc. Amer. Acad. Arts 25: 158. 1890. Sc. MEX (NL, Qro, SLP, Tam). HMF, QF. 900-1,300 m.

M. propinqua Hemsl. Biol. Cent.-Amer. Bot. 2(11): 337. 1882. Li. MEX (Chis, Col, Gro, Hgo, Mich, Nay, NL, Oax, Pue, QR, Tab, Tam, Ver, Yuc), MA (Bel, Gua, Hon). QF, SV, TDF, TEF, TSEF. 5-1,600 m.

M. rzedowskiana Juárez-Jaimes \& W.D. Stevens. Novon 5(4): 337-339. 1995. Li. MEX (Oax, Pue, Ver). HMF, SV. 1,400-2,120 m.

M. steyermarkii Woodson. Ann. Missouri Bot. Gard. 28(3): 285. 1941. Li. MEX (Chis), MA (Gua). HMF. 2,585 m.

M. tholiformis Juárez-Jaimes \& L.O. Alvarado-Cárdenas. Candollea 65(1): 64-67, fig. 2. 2010. Li. MEX (Nay). TSEF. NA.

M. trivirgulata Bartlett. Proc. Amer. Acad. Arts 44: 632. 1909. Li. MEX (Chis, Col, Gro, Jal, Mex, Oax, Sin), MA (CR, ES, Gua, Hon, Nic, Pan). SV, TDF, TEF, TSEF. 30-830 m.

M. tubularis L.O. Williams. Fieldiana, Bot. 32(4): 51-52. 1968. Li. MEX (Chis, Mex, Oax). HMF, TDF. 1,000-1,100 m.

M. veronicae W.D. Stevens. Novon 10(3): 246, 248-249. 2000. Li. MEX (Chis, Oax), MA (CR, ES, Gua, Hon, Nic). TDF. 370$740 \mathrm{~m}$.

M. zimapanica Hemsl. Biol. Cent.-Amer. Bot. 2(11): 338. 1882. Li. MEX (Chis, Gro, Hgo, Jal, Mex, Mich, Mor, Nay, Oax, Pue, Qro). SV, TDF, TSEF, XS. 200-2,400 m.

Matelea belizensis (Lundell \& Standl.) Woodson. Ann. Missouri Bot. Gard. 28: 232. 1941. Li. MEX (QR), MA (Bel). SV, TSEF. 700-900 m.

M. campechiana (Standl.) Woodson. Ann. Missouri Bot. Gard. 28: 234. 1941. Li. MEX (Cam, Chis, QR, Yuc), MA (Bel, Gua). SAV, TDF, TEF, TSEF. 0-1,500 m.

M. caudata (A. Gray) Woodson. Ann. Missouri Bot. Gard. 28: 233. 1941. Li. MEX (BCS, Chih, Son). TDF, XS. $160-740$ m.

M. chrysantha (Greenm.) Woodson. Ann. Missouri Bot. Gard. 28: 222. 1941. Li. MEX (DF, Gro, Jal, Mex, Mich, Mor, Oax, Pue, Qro, Tla). HMF, PQF, QF, XS. 1,060-3,000 m.

M. crassifolia Woodson. Ann. Missouri Bot. Gard. 28: 236. 1941. Li. MEX (Cam, QR, Yuc), MA (Bel). TDF, TSEF. 0-200 m.

M. crenata (Vail) Woodson. Ann. Missouri Bot. Gard. 28: 233. 1941. Li. MEX (Gto, Gro, Hgo, Jal, Mex, Mich, Mor, Oax, SLP, Sin). CF, QF, TDF, TSEF, XS. 1,900-2,250 m.

M. cyclophylla (Standl.) Woodson. Ann. Missouri Bot. Gard. 28: 233. 1941. Li. MEX (Chis, Col, Gro, Jal, Mex, Mich, Oax, Pue, Sin, Ver). TDF, TSEF. 210-1,480 m.

M. emmartinezii W.D. Stevens. Novon 15(4): 607-609, f. 3. 2005. Li. MEX (Chis). TDF, TEF. 800-900 m.

M. fulvida (F. Ballard) W.D. Stevens. Novon 9(4): 564. 1999. Li. MEX (Chis), MA (Bel, CR, Gua, Hon, Nic). TEF. $220-300$ m.

M. gonoloboides (B.L. Rob. \& Greenm.) Woodson. Ann. Missouri Bot. Gard. 28: 222. 1941. Li. MEX (Chis, Mex, Oax), MA (Gua, Hon). PQF, QF, TF. 200-1,800 m.

M. inconspicua (Brandegee) Woodson. Ann. Missouri Bot. Gard. 28: 222. 1941. Li. MEX (Oax, Pue, Ver). QF, SV, TF, XS. 1,900$2,850 \mathrm{~m}$.

M. magallanesii E.J. Lott. Phytologia 73: 277-280. 1992. Li. MEX (Jal). TDF. 50-250 m.

M. magnifolia (Pittier) Woodson. Ann. Missouri Bot. Gard. 28: 225. 1941. Li. MEX (Chis, Oax, Pue, Tab, Ver), MA (Bel, CR, ES, Gua, Hon, Nic, Pan). HMF, TEF. 70-1,200 m.

M. micrantha L.O. Williams. Fieldiana, Bot. 32(12): 184. 1970. Li. MEX (Cam, Chis), MA (Bel, Gua, Hon). SV, TDF, TEF. 120$430 \mathrm{~m}$.

M. ocellata W.D. Stevens. Novon 10(3): 252, 254. 2000. Li. MEX (Mich, Oax), MA (Nic). HMF, TDF, TSEF. 60-1,100 m.

M. picturata (Hemsl.) Woodson. Ann. Missouri Bot. Gard. 28: 233. 1941. Li. MEX (Chis, Qro), MA (Gua, Pan). HMF. 1,200$2,000 \mathrm{~m}$.

M. pueblensis (Brandegee) Woodson. Ann. Missouri Bot. Gard. 28: 223. 1941. Li. MEX (Oax, Pue). TDF. $800-940$ m.

M. purpusii (Brandegee) Woodson. Ann. Missouri Bot. Gard. 28: 223. 1941. Li. MEX (Gro, Oax, Pue). CF, XS. 2,100-2,300 m.

M. pusilliflora L.O. Williams. Fieldiana, Bot. 32(4): 55-56. 1968. Li. MEX (Cam, Chis), MA (Bel, Gua, Hon). TEF, TSEF. 180$240 \mathrm{~m}$.

M. reticulata (Engelm. ex A. Gray) Woodson. Ann. Missouri Bot. Gard. 28: 234. 1941. Li. MEX (Gro, Mex, Mor, Nay, Oax, Pue, Ver). CF, QF, XS. 1,850-2,300 m.

M. steyermarkii Woodson. Ann. Missouri Bot. Gard. 28: 278-279. 1941. Li. MEX (Chis), MA (Gua). HMF. 1,700-3,900 m.

M. sugillata W.D. Stevens. Novon 10(3): 252-256. 2000. Li. MEX (Oax, Qro, Ver), MA (Nic). HMF. 650-1,000 m.

M. velutina (Schtld.) Woodson. Ann. Missouri Bot. Gard. 28: 234. 1941. Li. MEX (Cam, Gto, Hgo, Pue, Qro, QR, SLP, Tab, Tam, Ver). HMF, SV, TDF, TSEF. 0-30 m.

Mesechites trifidus (Jacq.) Müll. Arg. Fl. Bras. 6(1): 151-152. 1860. Li. MEX (Cam, Chis, Gro, Oax, Tab, Tam, Ver), ANT, MA (Bel, CR, ES, Gua, Hon, Nic, Pan), SA. RF, SV, TDF, TEF, XS. 90-850 m. 
Appendix 2. Continuation.

Metalepis peraffinis (Woodson) Morillo. Ernstia, n.s. 1(2): 55. 1991. Li. MEX (Chis, Oax, Ver), MA (Bel, CR, Gua, Nic, Pan), SA. HMF, QF, TEF. 20-2,000 m.

Metastelma arizonicum A. Gray subsp. arizonicum. Proc. Amer. Acad. Arts 19: 85. 1883. Li. USA, MEX (Son). XS. 10-1,000 m.

M. arizonicum A. Gray subsp. chiapense (A. Gray) Liede \& Meve. Ann. Missouri Bot. Gard. 91(1): 42-43, f. 2a-f, 3. 2004. Li. MEX (Chis, Oax, Pue), MA (Bel, ES, Gua, Hon). CF, HMF, PQF. 915-2,250 m.

M. brachymischum W.D. Stevens. Novon 15(4): 623-625, f. 2. 2005. Li. MEX (Chis). TEF. 1,250-1,350 m.

M. californicum Benth. subsp. californicum. Bot. Voy. Sulphur 33-34, pl. 18. 1844. Li. MEX (BCS, Col, Nay, SLP, Sin, Son). XS. 0-100 m.

M. cuneatum Brandegee. Zoë 5: 216. 1905. Li. MEX (Sin, Son). XS. 0-25 m.

M. lanceolatum Schltr. Li. MEX (Chis, DF, Gro, Hgo, Jal, Mex, Mor, Oax, Pue, SLP, Yuc). TDF, XS. 1,000-2,200 m.

M. latifolium Rose. Contr. U.S. Natl. Herb. 1: 106. 1891. Li. MEX (Chih, Sin, Son). TDF. 250-1,200 m.

M. Iongicoronatum (L.O. Williams) Liede. Novon 7(1): 42. 1997. Li. MEX (Chis). PQF, TSEF. 1,300-1,700 m.

M. mexicanum (Brandegee) Fishbein \& R.A. Levin. Madroño 44(3): 270. 1997. Li. USA, MEX (Sin, Son). PQF, TDF. 900-1,550 m.

M. minutiflorum Wiggins. Contr. Dudley Herb. 3: 71. 1940. Li. MEX (BCS, Son). XS. 200-500 m.

M. miserum (L.O. Williams) W.D. Stevens. Phytologia 64(5): 335. 1988. Li. MEX (Chis), MA (Gua, Hon). HMF, PQF. 700-2,800 m.

M. palmeri S. Watson. Proc. Amer. Acad. Arts 18: 115. 1883. Li. USA, MEX (Chis, Coa, Gro, NL, Oax, Pue, Tam, Yuc), MA (Gua). TDF, XS. 500-2,540 m.

M. pringlei A. Gray. Proc. Amer. Acad. Arts 21: 396-397. 1886. Li. USA, MEX (Chih, Coa, Dgo, NL, SLP, Tam, Zac). CF, XS. 500$2,300 \mathrm{~m}$.

M. pubescens (Greenm.) W.D. Stevens. Phytologia 53(6): 405. 1983. Li. MEX (DF, Mex, Mich, Mor, Pue). TDF. 1,600-2,650 m.

M. schaffneri A. Gray. Proc. Amer. Acad. Arts 21: 396. 1886. Li. MEX (BCS, Dgo, Gro, Jal, Mor, Nay, Oax, Pue, SLP, Sin, Son). TDF, TF. 500-2,900 m.

M. schlechtendalii Decne. var. arenicola Liede \& Meve. Ann. Missouri Bot. Gard. 91(1): 77, f. 22d, 23. 2004. Li. MEX (Sin, Son). XS. 30-400 m.

M. schlechtendalii Decne. var. schlechtendalii. Prodr. 8: 513. 1844. Li. MEX (Cam, Chis, Mor, Oax, Pue, QR, Tab, Ver, Yuc), MA (Bel, ES, Gua, Hon, Nic). MG, PQF, SV, TDF, TSEF, XS. 0-2,200 m.

M. schlechtendalii Decne. var. trichophyllum (L.O. Williams) Liede \& Meve. Ann. Missouri Bot. Gard. 91(1): 77-78, f. 22a-f, 23. 2004. Li. MEX (Cam, Chis, Oax), MA (ES, Gua, Hon, Nic). PQF, TDF. 200-2,100 m.

M. sepium (Decne.) W.D. Stevens. Phytologia 64(5): 335. 1988. Li. MEX (Hgo, Oax), MA (Gua). NA.

M. stenomeres (Standl. \& Steyer.) W.D. Stevens. Phytologia 64(5): 335. 1988. Li. MEX (Oax), MA (Bel, Gua). CF. $250-300$ m.

M. turneri Liede \& Meve. Ann. Missouri Bot. Gard. 91(1): 80-82, f. 15, 25a-f. 2004. Li. MEX (NL, SLP, Tam). XS. $400-2,000$ m.

Microdactylon cordatum Brandegee. Zoë 5(11): 252-253. 1908. Li. MEX (Oax, Pue). XS. 1800-2060 m.

Odontadenia macrantha (Willd. ex Roem. \& Schult.) Markgr. Fl. Suriname 4(1): 461-462. 1937. Li. MEX (Chis, Oax), MA (Bel, CR, Gua, Hon, Nic, Pan), SA. RF, SAV, TDF, TEF. 300 m.

Orthosia cynanchoides W.D. Stevens. Novon 15(4): 633-635, f. 7. 2005. Li. MEX (Chis). HMF. 2,800 m.

O. glaberrima (Woodson) W.D. Stevens. Novon 15(4): 635. 2005. Li. MEX (Chis), MA (CR, ES, Gua, Nic, Pan). HMF, PQF, TEF. 700-2,500 m.

Oxypetalum cordifolium (Vent.) Schtlr. Symb. Antill. 1(2): 269. 1899. Li. MEX (Chis, Hgo, Jal, Oax, Qro, QR, SLP, Ver), ANT, MA (CR, Gua, Hon, Pan), SA. HMF. 40-2,000 m.

Pentalinon andrieuxii (Müll. Arg.) B.F. Hansen \& Wunderlin. Taxon 35(1): 168. 1986. Li. USA, MEX (Cam, Chis, Gro, Hgo, Mor, Oax, Pue, Qro, QR, SLP, Sin, Tab, Tam, Ver, Yuc), MA (Bel, ES, Gua, Hon, Nic). QF, SAV, SV, TDF, TSEF. 10-1,550 m.

Pinochia monteverdensis (J.F. Morales) M.E. Endress \& B.F. Hansen. Edinburgh J. Bot. 64(2): 272. 2007. Li. MEX (Oax), MA (CR, Gua). HMF, TEF. NA.

P. peninsularis (Woodson) M.E. Endress \& B.F. Hansen. Edinburgh J. Bot. 64(2): 272. 2007. Li. MEX (Cam, Tab), MA (Bel, Gua, Pan). TEF, TSEF. NA.

Polystemma guatemalense (Schltr.) W.D. Stevens. Novon 15(4): 618. 2005. Li. MEX (Chis, Col, Gro, Jal, Mex, Mich, Mor, Nay, Oax, Pue), MA (CR, ES, Gua, Hon, Nic). CF, SV, TDF. 1,000-1,320 m.

P. mirandae Lozada-Pérez. Novon 20(4): 429-431, f. 1. 2010. Li. MEX (Gro, Oax, Pue). SV, TDF. 1,000-1,300 m.

P. viridiflorum Decne. Prodr. 8: 602-603. 1844. Li. MEX (Chis, Mich, Oax, Ver), MA (Gua, Hon, Nic). SV, TDF, TSEF. 80-1,585 m.

Prestonia clandestina J.F. Morales. Novon 7(1): 60. 1997. Li. MEX (Chis, Ver). SAV, SV, TEF. 100-900 m.

P. longifolia (Sessé \& Moc.) J.F. Morales. Novon 6(3): 287. 1996. Li. MEX (Chis, Tab, Ver), MA (Bel, CR, ES, Gua, Nic, Pan), SA. SV, TEF, XS. 28-820 m.

P. mexicana A. DC. Prodr. 8: 429. 1844. Li. MEX (Cam, Chis, Col, Gto, Gro, Jal, Mich, Mor, Nay, Oax, Qro, QR, SLP, Sin, Tab, Tam, Ver, Yuc), MA (Bel, CR, ES, Gua, Hon, Nic, Pan), SA. QF, RF, TDF, TEF, TSEF, XS. 10-1,280 m. 
Appendix 2. Continuation.

P. portobellensis (Beurl.) Woodson. Ann. Missouri Bot. Gard. 18(4): 553-554. 1931. Li. MEX (Chis, Hgo, Qro, Tab, Ver), MA (Bel, CR, ES, Gua, Hon, Nic, Pan), SA. RF, SV, TDF, TEF, XS. 0-600 m.

P. speciosa Donn. Sm. Bot. Gaz. 27(6): 435. 1899. Li. MEX (Chis, Oax), MA (ES, Gua, Hon, Nic). CF, HMF, SV. 2,010 m.

Prosthecidiscus guatemalensis Donn. Sm. Bot. Gaz. 25(3): 150, t. 12. 1898. Li. MEX (Chis, Gro, Jal, Oax, Ver), MA (CR, ES, Gua, Hon, Nic). TDF. 30-1,000 m.

Rhabdadenia biflora (Jacq.) Müll. Arg. Fl. Bras. 6(1): 175. 1860. Li. USA, MEX (Cam, Chis, QR, Tab, Ver, Yuc), ANT, MA (Bel, CR, Gua, Hon, Nic, Pan), SA. MG, TDF, TSEF. 25 m.

Sarcostemma bilobum Hook. \& Arn. Bot. Beechey Voy. 438. 1841. Li. MEX (Cam, Chis, Col, Jal, Mex, Mich, Mor, Oax, Pue, QR, Ver, Yuc), MA (Bel, CR, ES, Gua, Hon, Nic, Pan), SA. SV, TDF, TEF, TSEF, XS. 660-900 m.

S. clausum (Jacq.) Schult. Syst. Veg. (ed. 15 bis) 6: 114-115. 1820. Li. USA, MEX (BCS, Cam, Chis, Chih, Col, Gro, Jal, Mich, Nay, Oax, QR, Sin, Son, Tab, Tam, Ver, Yuc, Zac), ANT, MA (Bel, CR, ES, Gua, Hon, Nic, Pan), SA. MG, SV, TDF. 0-1,200 m.

Tassadia obovata Decne. Prodr. 8: 579-580. 1844. Li. MEX (Chis, Oax, Ver), ANT, MA (Bel, CR, Nic, Pan), SA. TEF. 10-1,500 m.

Telminostelma foetidum (Cav.) Fontella \& E.A. Schwarz. Bol. Mus. Bot. Munic. 45: 6. 1981. Li. MEX (Chis, DF, Gto, Gro, Jal, Mex, Mich, Mor, Oax, Pue, Qro). TDF, XS. 200-2,330 m.

Thenardia chiapensis J.K. Williams. Brittonia 47(4): 403-407, f. 1-3. 1995. Li. MEX (Chis, Gro, Oax), MA (Hon). CF, PQF, RF. 910-1,700 m.

T. floribunda Kunth. Nov. Gen. Sp. (quarto ed.) 3: 210-211, pl. 240. 1818[1819]. Li. MEX (Col, Gro, Jal, Mex, Mich, Mor, Nay, Oax). HMF, QF. 1,200-1,900 m.

T. galeottiana Baill. Bull. Mens. Soc. Linn. Paris 2: 819. 1890. Li, Sc. MEX (Chis, Gro, Oax). HMF, PQF, SV, TDF. 1,070-1,690 m.

Thoreauea aberrans J.F. Morales. Brittonia 57(3): 260-262, f. 1-2. 2005. Li. MEX (Ver). TEF. 1,350 m.

T. guerrerensis Diego \& Lozada-Pérez. Novon 16(3): 332-334, f. 1. 2006. Li. MEX (Gro). HMF, PQF, QF. 1,800-2,650 m.

T. paneroi J.K. Williams. Lundellia 5: 47-57, f. 1-2. 2002. Li. MEX (Oax). HMF, PQF. 2,400-2,600 m.

Tintinnabularia gratissima J.F. Morales. Novon 6(4): 392-394, f. 1. 1996. Li. MEX (Ver). SV, TEF. 1,400-1,600 m.

T. mortonii Woodson. Ann. Missouri Bot. Gard. 23(2): 389-391, t. 7. 1936. Li. MEX (Chis, Oax), MA (Gua). HMF, SV, TEF. 600$2,600 \mathrm{~m}$.

\section{Arecaceae (1/1)}

Desmoncus orthacanthos Mart. Hist. Nat. Palm. 2(3): 87, t. 69, 98. 1824. Li, Sc. MEX (Cam, Chis, Oax, QR, Tab, Ver, Yuc), ANT, MA (Bel, CR, ES, Gua, Hon, Nic, Pan), SA. RF, TEF, TSEF. 150-200 m.

\section{Aristolochiaceae (1/18)}

Aristolochia anguicida Jacq. Enum. Syst. PI. 30. 1760. Li. MEX (Chis, Mich, Oax, Yuc), ANT, MA (CR, ES, Gua, Hon, Nic, Pan), SA. SV, TDF. 0-1,000 m.

A. asclepiadifolia Brandegee. Univ. Calif. Publ. Bot. 6(8): 178. 1915. Li, Sc. MEX (Ver). QF, RF, TDF, TSEF. 100-1,000 m.

A. bullata Pfeifer. Ann. Missouri Bot. Gard. 53(2): 134-135, f. 8. 1966. Li. MEX (Gro, Ver), MA (Gua). TDF. 25-100 m.

A. cardiantha Pfeiffer. Taxon. Rev. Pentandrous Sp. Aristolochia 21, f. 3. 1970. Li. MEX (Gro). TDF. 500 m.

A. carterae Pfeifer. Ann. Missouri Bot. Gard. 53(2): 178-179, f. 52. 1966. Li. MEX (Jal, Nay). RF, TSEF. 0-30 m.

A. chiapensis J.F. Ortega \& R.V. Ortega. Acta Bot. Mex. 44: 2-4, f. 1. 1998. Li. MEX (Chis). TEF. 100-350 m.

A. glossa Pfeifer. Ann. Missouri Bot. Gard. 53(2): 180-181, f. 54. 1966. Li. MEX (Mich). RF, TDF, TSEF. 300-1,000 m.

A. grandiflora Sw. Prodr. 126. 1788. Li. MEX (Chis, Oax, Tab, Ver, Yuc), ANT, MA (Bel, CR, ES, Gua, Hon, Nic, Pan), SA. SV, RF, TEF. 0-1,500 m.

A. inflata Kunth. Nov. Gen. Sp. (quarto ed.) 2: 145-146, t. 111. 1817. Li. MEX (Chis, Gro), MA (CR, Gua, Hon, Nic, Pan), SA. TDF, TSEF. 0-1,500 m.

A. leuconeura Linden. Belgique Hort. 8: 165. 1858. Li. USA, MEX (Ver), MA (Bel, CR, Hon, Pan), SA. TEF. 0-1,000 m.

A. malacophylla Standl. Proc. Biol. Soc. Wash. 33(12): 65-66. 1920. Li, Sc. MEX (Chis, Col, Jal, Mich), MA (Gua, Hon). PQF. 1,500-1,800 m.

A. maxima Jacq. Enum. Syst. PI. 30. 1760. Li. USA, MEX (Cam, Chis, Tab, Yuc), ANT, MA (Bel, CR, ES, Gua, Hon, Nic, Pan), SA. SV, TEF, TSEF. 0-1,200 m.

A. odoratissima L. Sp. Pl. (ed. 2) 2: 1362. 1763. Li. MEX (Chis, Jal, Mich, Oax, Tab, Ver), ANT, MA (Bel, CR, Gua, Hon, Nic, Pan), SA. SV, TEF, TSEF. 500-750 m.

A. ovalifolia Duch. Ann. Sci. Nat. Bot. sér. 4, 2: 50-51. 1854. Li. MEX (Chis, Oax, Tab, Ver, Yuc), MA (Bel). RF, SV, TEF. 0-500 m.

A. paracleta Pfeifer. Ann. Missouri Bot. Gard. 53(2): 135-136, f. 9. 1966. Li. MEX (Chis), MA (Gua). NA.

A. pilosa Kunth. Nov. Gen. Sp. (quarto ed.) 2: 146-147, t. 113. 1817. Li. MEX (Chis, Gro, Mich, Mor, Oax, Tab, Ver), MA (Bel, CR, Gua, Hon, Nic, Pan), SA. SV, TDF, TEF. 10-50 m.

A. taliscana Hook. \& Arn. Bot. Beechey Voy. 309. 1841 [1838]. Li. MEX (Col, Jal, Mich, Nay, Sin). RF, TDF. $700-900$ m.

A. veracruzana J.F. Ortega. Biótica 12: 209. 1987. Li. MEX (Ver). QF, RF, SV, TEF. 150-650 m. 


\section{Asteraceae (23/61)}

Archibaccharis albescens (J.D. Jacks.) G.L. Nesom. Phytologia 65(2): 124. 1988. Li. MEX (Oax). PQF, QF. 1,900-2,900 m.

A. flexilis (S.F. Blake) S.F. Blake. J. Wash. Acad. Sci. 17(3): 60. 1927. Li. MEX (Chis), MA (CR, ES, Gua, Nic). CF, HMF, SV, TEF. 500-3,000 m.

A. hirtella (DC.) Heering. Jahrb. Hamburg. Wiss. Anst. 21: Beih. 3: 41. 1904. Li. MEX (Chis, DF, Gro, Hgo, Mex, Mich, Mor, Oax), MA (ES). CF, HMF, PQF, QF. 600-3,200 m.

A. salmeoides (S.F. Blake) S.F. Blake. J. Wash. Acad. Sci. 17(3): 61. 1927. Li. MEX (Chis, Oax, Ver), MA (Gua). 1,300-1,900 m.

A. schiedeana (Benth.) J.D. Jacks. Phytologia 28(3): 297. 1974. Li, Sc. MEX (Chis, Gro, Hgo, Jal, Mex, Mich, Mor, Nay, Oax, Pue, SLP, Ver), MA (CR, ES, Gua, Hon, Pan). HMF, PQF, QF, TEF. 600-2,600 m.

A. taeniotricha (S.F. Blake) G.L. Nesom. Phytologia 65(2): 125. 1988. Li. MEX (Chis), MA (ES, Gua). HMF, PQF. 900-3,000 m.

A. veracruzana G.L. Nesom. Phytologia 65(2): 127-128. 1988. Li, Sc. MEX (Ver). HMF, QF. 1,700-2,260 m.

Baccharis trinervis Pers. Syn. PI. 2: 423. 1807. Sc. MEX (Cam, Chis, Col, Gro, Hgo, Jal, Mich, Mor, Nay, Oax, Pue, QR, Qro, SLP, Tab, Ver, Yuc), MA (Bel, CR, ES, Gua, Hon, Nic, Pan), SA. HMF, PQF, SV, TEF, TSEF. 14-1,990 m.

Bidens holwayi Sherff \& S.F. Blake. Bot. Gaz. 64: 39. 1917. Li. MEX (Chis), MA (Gua). 2,700 m.

B. reptans (L.) G. Don var. urbanii (Greenm.) O.E. Schulz. Hort. Brit. (ed. 3) 360. 1839. Li, Sc. MEX (Cam, Col, Gro, Jal, Mich, Oax, QR, Sin, Ver, Yuc), ANT, MA (Bel, ES, Gua, Hon, Nic, Pan), SA. SV, TDF, TSEF. 0-1,650 m.

Calea jamaicensis (L.) L. Sp. PI. (ed. 2) 2: 1179. 1763. Li, Sc. MEX (Cam, Chis, Nay, Oax, QR, Tab, Ver, Yuc), ANT, MA (Bel, CR, Gua, Hon, Nic, Pan). SV, TDF, TEF, TSEF. 20-500 m.

Chromolaena odorata (L.) R.M. King \& H. Rob. Phytologia 20(3): 204. 1970. Sc. USA, MEX (Cam, Chis, Col, Gro, Hgo, Jal, Mich, Nay, NL, Oax, QR, Sin, Tab, Tam, Ver, Yuc), ANT, MA (Bel, CR, ES, Gua, Hon, Nic, Pan), SA. RF, TDF. 800-1,700 m.

Critonia billbergiana (Beurl.) R.M. King \& H. Rob. Phytologia 22(1): 48. 1971. Li. MEX (Ver), MA (Bel, CR, Gua, Nic, Pan), SA. TEF. $160 \mathrm{~m}$.

C. campechensis (B.L. Rob.) R.M. King \& H. Rob. Phytologia 22(1): 48. 1971. Li. MEX (Cam, QR, Yuc), MA (Bel, Gua). TSEF. 200-230 m.

C. yashanalensis (Whittem.) R.M. King \& H. Rob. Phytologia 69(2): 89. 1990. Sc. MEX (Chis). HMF, TEF. 1,800-2,460 m.

Critoniopsis pugana (S.B. Jones \& Stutts) H. Rob. Proc. Biol. Soc. Wash. 106(3): 620. 1993. Li, Sc. MEX (Jal). HMF, PQF. 1,500$2,000 \mathrm{~m}$.

Hidalgoa ternata La Llave. Nov. Veg. Descr. 1: 15. 1824. Li. MEX (Chis, Oax, Pue, Tab, Ver), MA (CR, Gua, Pan), SA. HMF, PQF, TEF. 150-1,900 m.

Jungia ferruginea L. f. Suppl. Pl. 390. 1781[1782]. Li, Sc. MEX (Chis), MA (CR, ES, Gua, Pan), SA. CF, HMF. 1,400-3,100 m.

Lepidaploa canescens (Kunth) Cass. var. canescens. Proc. Biol. Soc. Wash. 103(2): 483. 1990. Sc. MEX (Chis, Col, Gro, Hgo, Jal, Nay, Oax, QR, Tab, Yuc), ANT, MA (Bel, CR, ES, Gua, Hon, Nic, Pan), SA. CF, HMF, PQF, SV, TDF. 0-2,500 m.

L. tortuosa (L.) H. Rob. Proc. Biol. Soc. Wash. 103(2): 495. 1990. Sc. MEX (Chis, Hgo, Oax, Pue, Tab, Ver), MA (Bel, CR, ES, Gua, Hon, Nic, Pan). CF, PQF, SV, TDF. 5-1,900 m.

Mikania cordifolia (L. f.) Willd. Sp. PI. 3(3): 1746. 1803. Li. USA, MEX (Cam, Chis, Col, Gro, Hgo, Jal, Mex, Mich, Nay, Oax, Pue, Qro, QR, SLP, Tab, Tam, Ver, Yuc), ANT, MA (Bel, CR, ES, Gua, Hon, Nic, Pan), SA. HMF, TEF, TSEF. 0-1,000 m.

M. globosa J.M. Coult. Enum. PI. Guatem. 4: 77. 1895. Li. MEX (Chis), MA (Gua). HMF, TEF. 1,500-2,000 m.

M. gonzalezii B.L. Rob. \& Greenm. Proc. Boston Soc. Nat. Hist. 29: 107. 1899. Li. MEX (Chis, Oax, Tab, Ver), MA (CR, Gua, Nic, Pan), SA. HMF. 945-2,100 m.

M. guaco Bonpl. Pl. Aequinoct. 2(13): 84, t. 105. 1809[1811]. Li. MEX (Chis, Oax, Tam, Ver), MA (Bel, CR, Gua, Hon, Nic, Pan), SA. TEF. NA.

M. hookeriana DC. Prodr. 5: 195. 1836. Li, Sc. MEX (Chis, Oax, Tab, Ver), ANT, MA (Bel, CR, Gua, Hon, Nic, Pan), SA. HMF, TEF. 150-2,800 m.

M. leiostachya Benth. PI. Hartw. 201. 1845. Li, Sc. MEX (Chis, Oax, Ver), MA (Bel, CR, Gua, Hon, Nic, Pan), SA. SV, TEF. 152$470 \mathrm{~m}$.

M. micrantha Kunth. Nov. Gen. Sp. (folio ed.) 4: 105. 1820[1818]. Li, Sc. MEX (Cam, Chis, Col, Gro, Hgo, Jal, Mich, Nay, NL, Oax, Pue, QR, SLP, Sin, Son, Tab, Tam, Ver, Yuc), ANT, MA (Bel, ES, Gua, Hon, Nic, Pan), SA. RF, SV, TDF, TEF, TSEF. 102,600 m.

M. neei W.C. Holmes. Phytologia 58: 165. 1985. Li. MEX (Ver). TEF. 400 m.

M. tonduzii B.L. Rob. Proc. Boston Soc. Nat. Hist. 31: 256. 1904. Li. MEX (Chis, Gro, Oax, Ver), MA (CR, Nic, Pan). HMF, TEF. 470-1,070 m.

M. vitifolia DC. Prodr. 5: 202. 1836. Li. MEX (Chis, Oax, Ver), MA (CR, Nic), SA. TEF. 100-480 m.

Neohintonia monantha (Sch. Bip.) R.M. King \& H. Rob. Phytologia 22: 144. 1971. Sc. MEX (Col, Dgo, Gro, Jal, Mex, Mich, Nay, Oax, Sin, Son). HMF, QF, RF, TDF, TSEF. 60-1,700 m.

Neomirandea araliifolia (Less.) R.M. King \& H. Rob. Phytologia 19(5): 307. 1970. He. MEX (Chis, Jal, Oax, Tab, Ver), MA (Bel, CR, Gua, Hon, Pan), SA. HMF, QF. 1,600-1,850 m. 
Appendix 2. Continuation.

Oteiza scandens Panero, Villaseñor \& Medina. Contr. Univ. Michigan Herb. 19: 171-193. 1993. Li, Sc. MEX (Oax). HMF. 2,500$2,900 \mathrm{~m}$.

Otopappus acuminatus S. Watson. Proc. Amer. Acad. Arts 26: 140-141. 1891. Li, Sc. MEX (Jal). QF, TDF. 1,200-1,700 m.

O. brevipes B.L. Rob. Proc. Amer. Acad. Arts 44(21): 621-622. 1909. Li, Sc. MEX (Chis), MA (ES, Gua, Hon, Nic). HMF, PQF. 712-2,100 m.

O. curviflorus (R. Br.) Hemsl. Biol. Cent.-Amer. Bot. 2(8): 191. 1881. Li, Sc. MEX (Cam, Chis, Oax, QR, Tab, Ver), MA (Bel, CR, Gua, Hon, Nic). SV, TEF. 20-1,300 m.

O. epaleaceus Hemsl. Biol. Cent.-Amer. Bot. 2(8): 191. 1881. Li, Sc. MEX (Gro, Mex, Mich, Mor, Oax, Pue). QF, TDF, TSEF. 25$1,950 \mathrm{~m}$.

O. guatemalensis (Urb.) R.L. Hartm. \& Stuessy. Syst. Bot. 8(2): 206. 1983. Li, Sc. MEX (Cam, Chis, QR, Yuc), MA (Bel, Gua). RF, TDF, TSEF. 0-300 m.

O. koelzii McVaugh. Contr. Univ. Michigan Herb. 9(4): 425, f. 32. 1972. Li, Sc. MEX (Col, Jal, Mich). TDF, TSEF. 50-600 m.

O. mexicanus (Rzed.) H. Rob. Wrightia 6(3): 44. 1979. Li. MEX (Gro, Oax). 800-840 m.

O. microcephalus S.F. Blake. J. Bot. 53: 232. 1915. Li, Sc. MEX (Col, Gro, Jal, Mich, Nay, Oax). TDF, TSEF. 0-1,000 m.

O. robustus Hemsl. Biol. Cent.-Amer. Bot. 2(8): 191-192, t. 49. 1881. Li, Sc. MEX (Ver). TDF. 1,000 m.

O. scaber S.F. Blake. Contr. U.S. Natl. Herb. 22(8): 636-637. 1924. Li, Sc. MEX (Cam, Chis, QR), MA (Bel, Gua). PQF, TDF. 100$1,400 \mathrm{~m}$.

O. tequilanus (A. Gray) B.L. Rob. Proc. Amer. Acad. Arts 44: 622. 1909. Li, Sc. MEX (Col, Gro, Jal, Mich, Nay, Sin). PQF, QF, RF, TDF, TSEF. 0-3,700 m.

O. verbesinoides Benth. Hooker's Icon. PI. 12: 47-48, t. 1153. 1876[1873]. Li, Sc. MEX (Chis, Oax, Ver), MA (Bel, CR, ES, Gua, Hon, Nic). HMF, QF, TEF. 20-2,400 m.

Pentacalia wilburii H. Rob. Phytologia 40(1): 44-45, unnumbered figs. pg 49, 50. 1978. Li. MEX (Chis, Oax, Pue), MA (CR, Gua). HMF, PQF, QF. 550-2,600 m.

Perymeniopsis ovalifolia (A. Gray) H. Rob. Phytologia 40: 496. 1978. Sc. MEX (Hgo, Oax, Pue, Qro, SLP, Tam, Ver). HMF, QF, SV, TDF. 800-1,850 m.

Piptocarpha poeppigiana (DC.) Baker. FI. Bras. 6(2): 131. 1873. Li, Sc. MEX (Chis, Ver), MA (Bel, CR, Gua, Hon, Nic, Pan), SA. HMF, TEF. 15-2,000 m.

Pseudogynoxys chenopodioides (Kunth) Cabrera. Brittonia 7(2): 56. 1950. Li. MEX (Cam, Chis, Hgo, Oax, Qro, QR, SLP, Tab, Tam, Ver, Yuc), ANT, MA (Bel, CR, ES, Gua, Hon, Nic, Pan), SA. HMF, MG, SV, TDF, TEF, TSEF. 500-1,000 m.

P. haenkei (DC.) Cabrera. Brittonia 7(2): 54. 1950. Li. MEX (Chis, Hgo, Oax), MA (ES, Gua, Hon, Nic). HMF, TDF, TEF, TF, TSEF. $180-2,100 \mathrm{~m}$.

Salmea scandens (L.) DC. Cat. PI. Horti Monsp. 141. 1813. Li, Sc. MEX (Chis, Gro, Hgo, Mex, Qro, SLP, Tab, Tam, Ver), ANT, MA (Bel, CR, ES, Gua, Hon, Nic, Pan), SA. HMF, PQF, SV, TSEF. 150-2,600 m.

Sinclairia broomeae H. Rob. Phytologia 33(4): 287. 1976. Li. MEX (Gro, Jal). TDF. NA.

S. caducifolia (B.L. Rob. \& Bartlett) Rydb. N. Amer. Fl. 34(4): 299. 1927. Li, Sc. MEX (Gro, Jal). TDF, TSEF. NA.

S. deamii (B.L. Rob. \& Bartlett) Rydb. N. Amer. Fl. 34(4): 299. 1927. Li, Sc. MEX (Cam, Chis, Tab), MA (Bel, ES, Gua, Hon). TDF, TEF. 100-1,200 m.

S. deppeana (Less.) Rydb. N. Amer. Fl. 34(4): 300. 1927. Li, Sc. MEX (Hgo, Oax). HMF, PQF, TSEF. 1,430-1,800 m.

S. discolor Hook. \& Arn. Bot. Beechey Voy. (10): 433. 1841. Sc. MEX (Chis, Oax, Pue, Ver), MA (Bel, CR, ES, Gua, Hon, Nic). CF, HMF, SV. 170-2,700 m.

S. hypochlora (S.F. Blake) Rydb. N. Amer. FI. 34(4): 301. 1927. Sc. MEX (Chis), MA (Gua). 500-1,000 m.

S. polyantha (Klatt) Rydb. N. Amer. Fl. 34(4): 299. 1927. Li, Sc. MEX (Chis, Oax, Ver), MA (Bel, CR, Gua, Hon, Nic, Pan), SA. HMF, TEF. 0-1,800 m.

S. sublobata (B.L. Rob.) Rydb. N. Amer. Fl. 34(4): 297. 1927. Sc. MEX (Chis, Oax), MA (ES, Gua, Hon, Nic). PQF, TDF. 950 m.

Tuxtla pittieri (Greenm.) Villaseñor \& Strother. Syst. Bot. 14(4): 537. 1989. Li. MEX (Ver), MA (CR). TEF. $200-280$ m.

Zexmenia serrata La Llave. Nov. Veg. Descr. 1: 13. 1824. Sc. MEX (Chis, Oax, Tab, Ver), MA (Bel, Gua, Hon). PQF, SV, TEF, TSEF. 10-1,990 m.

\section{Bignoniaceae (16/41)}

Adenocalymma inundatum Mart. ex DC. Prodr. 9: 201. 1845. Li. MEX (Cam, Chis, Col, Gro, Jal, Mex, Mich, Mor, Nay, Oax, Pue, Sin, Tab, Ver), ANT, MA (Bel, CR, ES, Gua, Hon, Nic, Pan), SA. RF, SV, TDF, TEF, TSEF. 0-1,330 m.

A. sousae A.H. Gentry. FI. Veracruz 24: 22. 1982. Li. MEX (Tab, Ver). MG. 0-5 m.

Amphilophium buccinatorium (DC.) L.G. Lohmann. Ann. Missouri Bot. Gard. 99(3): 401. 2014. Li. MEX (Chis, Jal, Mor), MA (Gua). HMF. 1,700-2,400 m. 
Appendix 2. Continuation.

A. crucigerum (L.) L.G. Lohmann. Nuevo Cat. Fl. Vasc. Venezuela 270. 2008. Li. MEX (Cam, Chis, Col, Gto, Gro, Jal, Mex, Mich, Mor, Nay, Oax, Pue, Qro, SLP, Sin, Tab, Tam, Ver, Yuc), ANT, MA (Bel, CR, ES, Gua, Hon, Nic), SA. QF, RF, SV, TDF, TEF, TSEF. 0-1,500 m.

A. laxiflorum (DC.) L.G. Lohmann. Ann. Missouri Bot. Gard. 99(3): 405. 2014. Li. MEX (Chis, Gto, Gro, Jal, Oax, Pue, Ver), MA (Nic). HMF, QF, TDF, TSEF, XS. 1,100-2,050 m.

A. paniculatum (L.) Kunth Nov. Gen. Sp. (quarto ed.) 3: 149. 1818[1819]. Li. MEX (Cam, Chis, Col, Gro, Hgo, Jal, Mex, Mich, Mor, Nay, Oax, Pue, Qro, QR, SLP, Sin, Tab, Tam, Ver, Yuc), MA (Bel, CR, ES, Gua, Hon, Nic, Pan), SA. CF, HMF, QF, RF, SV, TDF, TEF, TSEF. 0-2,200 m.

Anemopaegma chrysanthum Dugand. Caldasia 4(19): 307-309. 1947. Li. MEX (Chis, Oax, Ver), MA (Bel, CR, Hon, Nic, Pan), SA. RF, TDF, TEF, TSEF. 0-1150 m.

A. chrysoleucum (Kunth) Sandwith. Lilloa 3: 459-460. 1938. Li. MEX (Ver), MA (Bel, CR, Gua, Hon, Nic, Pan), SA. RF, SV, TEF. 0-500 m.

A. puberulum (Seibert) Miranda. Anales Inst. Biol. Univ. Nac. México 24(1): 93-94. 1953. Li. MEX (Chis, Oax, Ver), MA (CR, ES, Gua, Hon, Pan), SA. TDF. 0-1,492 m.

Bignonia aequinoctialis L. Sp. PI. 2: 623. 1753. Li. MEX (Chis, Col, Gro, Jal, Mich, Nay, Oax, Sin, Tab, Ver, Yuc), ANT, MA (Bel, CR, ES, Gua, Hon, Nic, Pan), SA. CF, SV, TDF, TEF, TSEF. 0-1,600 m.

B. binata Thunb. PI. Bras. 3: 35. 1821. Li. MEX (Cam, Chis, Gro, Jal, Oax, QR, Tab, Ver), MA (Bel, CR, ES, Gua, Hon, Nic, Pan), SA. MG, TDF, TEF, TSEF. 0-670 m.

B. diversifolia Kunth. Nov. Gen. Sp. (quarto ed.) 3: 133-134. 1818. Li. MEX (Cam, Chis, Gro, Jal, Mich, Oax, QR, Tab, Yuc), ANT, MA (Bel, CR, ES, Gua, Hon, Nic, Pan), SA. TDF, TEF, TSEF. 50-1,000 m.

B. hyacinthina (Standl.) L.G. Lohmann. Nuevo Cat. Fl. Vasc. Venez. 272. 2008. Li. MEX (Chis, Oax, Ver), MA (Bel, Gua, Nic, Pan), SA. TEF. 100-300 m.

B. neoheterophylla L.G. Lohmann. Ann. Missouri Bot. Gard. 99(3): 420. 2014. Li. MEX (Cam, Chis, Gro, Jal, Nay, Oax, QR, Tab, Ver, Yuc), ANT, MA (Bel, CR, ES, Gua, Hon, Nic, Pan), SA. QF, SV, TDF, TEF. 0-833 m.

B. potosina (K. Schum. \& Loes.) L.G. Lohmann. Ann. Missouri Bot. Gard. 99(3): 421. 2014. Li. MEX (Cam, Chis, Oax, Pue, Qro, QR, SLP, Tab, Ver, Yuc), MA (Bel, CR, ES, Gua, Hon, Nic). RF, SV, TDF, TSEF. 0-450 m.

Callichlamys latifolia (Rich.) K. Schum. Nat. Pflanzenfam. 4(3b): 223. 1894. Li. MEX (Chis, Oax, Ver), MA (Bel, CR, Gua, Hon, Nic, Pan), SA. TDF, TEF. 0-200 m.

Cuspidaria inaequalis (DC. ex Splitg.) L.G. Lohmann. Nuevo Cat. FI. Vasc. Venez. 272. 2008. Li. MEX (Cam, Chis, Tab, Ver), MA (Bel), SA. MG, TEF. 100-200 m.

Dolichandra quadrivalvis (Jacq.) L.G. Lohmann. Nuevo Cat. Fl. Vasc. Venez. 273. 2008. Li. MEX (Chis, Col, Gro, Hgo, Jal, Oax, Pue, Qro, QR, SLP, Tam, Ver, Yuc), MA (CR, ES, Gua, Hon, Nic), SA. RF, SV, TDF, TEF, TSEF. 0-1,400 m.

D. uncata (Andrews) L.G. Lohmann. Nuevo Cat. Fl. Vasc. Venez. 273. 2008. Li. MEX (QR, Tab, Ver), MA (Bel, Gua, Hon, Nic, Pan), SA. RF, TEF. 0-100 m.

D. unguis-cati (L.) L.G. Lohmann. Nuevo Cat. Fl. Vasc. Venez. 273. 2008. Li. MEX (BCS, Cam, Chis, Gto, Gro, Jal, Mex, Mich, Mor, Oax, Pue, Qro, QR, SLP, Sin, Son, Tab, Tam, Ver, Yuc), ANT, MA (Bel, CR, ES, Gua, Hon, Nic, Pan), SA. QF, SV, TDF, TEF, TSEF. 0-1,850 m.

Fridericia candicans (Rich.) L.G. Lohmann. Ann. Missouri Bot. Gard. 99(3): 433. 2014. Li. MEX (Oax, Ver), MA (Bel, CR, Hon, Pan), SA. TEF, TSEF. 5-900 m.

F. chica (Bonpl.) L.G. Lohmann. Ann. Missouri Bot. Gard. 99(3): 434. 2014. Li. MEX (Chis, Oax, Ver), MA (Bel, ES, Gua, Nic, Pan), SA. TDF, TEF. 5-900 m.

F. dichotoma (Jacq.) L.G. Lohmann. Ann. Missouri Bot. Gard. 99(3): 436-437. 2014. Li. MEX (Chis, Col, Gro, Jal, Mex, Oax, Ver), MA (Bel, CR, Nic, Pan), SA. TDF, TEF, TSEF. 0-850 m.

F. floribunda (Kunth) L.G. Lohmann. Ann. Missouri Bot. Gard. 99(3): 438. 2014. Li. MEX (Cam, Chis, Oax, QR, Yuc), MA (Bel, Gua, Hon). RF, TDF, TEF, TSEF, XS. 0-70 m.

F. florida (DC.) L.G. Lohmann. Ann. Missouri Bot. Gard. 99(3): 438. 2014. Li. MEX (Oax, Ver), MA (Bel, CR, Nic, Pan), SA. TEF, TSEF. 20-340 m.

F. mollissima (Kunth) L.G. Lohmann. Ann. Missouri Bot. Gard. 99(3): 440. 2014. Li, Sc. MEX (Chis, Gro, Mich, Oax), MA (ES, Gua, Nic, Pan), SA. MG, TDF. 50-1,000 m.

F. patellifera (Schltdl.) L.G. Lohmann. Ann. Missouri Bot. Gard. 99(3): 442. 2014. Li. MEX (Cam, Chis, Col, Gro, Jal, Mich, Nay, Oax, QR, Tab, Ver), MA (Bel, CR, ES, Gua, Hon, Nic, Pan), SA. SAV, SV, TDF, TEF, TSEF. 0-1,270 m.

F. podopogon (DC.) L.G. Lohmann. Ann. Missouri Bot. Gard. 99(3): 443. 2014. Li. MEX (Cam, Chis, QR, Yuc), MA (Bel). TDF, TEF, TSEF. 10-275 m.

F. pubescens (L.) L.G. Lohmann. Ann. Missouri Bot. Gard. 99(3): 443-444. 2014. Li. MEX (Cam, Chis, Pue, Qro, QR, SLP, Tab, Tam, Ver, Yuc), MA (Bel, CR, Gua, Pan), SA. QF, TDF, TEF, TSEF. 100-1,300 m. 
Appendix 2. Continuation.

F. schumanniana (Loes.) L.G. Lohmann. Ann. Missouri Bot. Gard. 99(3): 444. 2014. Li. MEX (Cam, Chis, Oax, QR, Ver), MA (Bel, CR, Hon, Nic, Pan), SA. TDF, TEF, TSEF. 20-350 m.

F. viscida (Donn. Sm.) L.G. Lohmann. Ann. Missouri Bot. Gard. 99(3): 446-447. 2014. Li. MEX (Chis, Col, Gro, Jal, Mor, Oax), MA (Bel, Gua, Nic). RF, TDF, TSEF. 10-1,350 m.

Lundia puberula Pittier. Contr. U.S. Natl. Herb. 18(6): 258. 1917. Li. MEX (Cam, Oax, Ver), MA (Bel, CR, Nic, Pan), SA. TEF. 0-50 m.

Mansoa hymenaea (DC.) A.H. Gentry. Ann. Missouri Bot. Gard. 66(4): 782. 1979[1980]. Li. MEX (Cam, Chis, Gro, Nay, Oax, SLP, Sin, Tab, Tam, Ver, Yuc), MA (Bel, CR, ES, Nic, Pan), SA. RF, TDF, TSEF. 0-1,000 m.

M. verrucifera (Schltdl.) A.H. Gentry. Ann. Missouri Bot. Gard. 63(1): 62. 1976. Li. MEX (Cam, Chis, Gro, Oax, QR, Tab, Ver, Yuc), MA (CR, Hon, Nic, Pan), SA. RF, TDF, TEF, TSEF. 100-1,100 m.

Martinella obovata (Kunth) Bureau \& K. Schum. FI. Bras. 8(2): 161. 1896. Li. MEX (Oax, Tab), MA (Bel, CR, Gua, Hon, Pan), SA. TEF. 350- $\mathrm{m}$.

Paragonia pyramidata (Rich.) Bureau var. pyramidata. Kongel. Danske Vidensk. Selsk. Skr. Naturvidensk. Math. Afd. ser. 6, 6(3): 422. 1892. Li. MEX (Cam, Chis, Col, Oax, QR, Tab, Ver, Yuc), ANT, MA (Bel, CR, ES, Gua, Hon, Nic, Pan), SA. SV, TDF, TEF, TSEF. 0-1,100 m.

Stizophyllum riparium (Kunth) Sandwith. Lilloa 3: 462. 1938. Li. MEX (Cam, Chis, Oax, QR, Tab, Ver, Yuc), MA (Bel, CR, ES, Gua, Pan), SA. RF, SV, TDF, TEF, TSEF. 0-1,100 m.

Tanaecium caudiculatum (Standl.) L.G. Lohmann. Ann. Missouri Bot. Gard. 99(3): 464. 2014. Li. MEX (Oax, Ver), MA (Bel, Gua). SV, TEF. 100-150 m.

T. tetragonolobum (Jacq.) L.G. Lohmann. Nuevo Cat. Fl. Vasc. Venez. 274. 2008. Li. MEX (Cam, Chis, Oax, QR, Tab, Ver, Yuc), MA (Bel, Nic, Pan), SA. TEF, TSEF. 0-150 m.

Tynanthus guatemalensis Donn. Sm. Bot. Gaz. 18(1): 6. 1893. Li. MEX (Cam, Chis, Oax, QR, Tab, Ver, Yuc), MA (Bel, Gua). SV, TEF, TSEF. 100-350 m.

Xylophragma seemannianum (Kuntze) Sandwith. Kew Bull. 8(4): 469-470. 1953[1954]. Li. MEX (Cam, Chis, Col, Jal, Mich, Oax, Tab, Ver), MA (Bel, CR, ES, Gua, Hon, Nic, Pan), SA. TDF, TEF, TSEF. 100-1,100 m.

\section{Boraginaceae (3/9)}

Cordia spinescens L. Mant. PI. 2: 206. 1771. Sc. MEX (Chis, Col, Gro, Hgo, Jal, Mich, Nay, Oax, Sin, Tab, Ver), MA (Bel, CR, ES, Gua, Hon, Nic, Pan), SA. HMF, PQF, QF, SV, TEF, TSEF. 3-1,900 m.

Rochefortia lundellii Camp. Contr. Univ. Michigan Herb. 7: 47-48. 1942. Li, Sc. MEX (Cam, Chis, QR, Ver), MA (Bel, CR, Gua, Hon). PQF, SV, TDF, TEF. 50-900 m.

Tournefortia bicolor Sw. Prodr. 40. 1788. Li, Sc. MEX (Chis, Gro, Oax, Tab, Ver), MA (Bel, CR, Gua, Hon, Nic, Pan), SA. HMF, MG, TEF. 0-1,700 m.

T. cuspidata Kunth. Li, Sc. MEX (Oax, Ver), MA (Nic), SA. RF, TEF. 0-1,500 m.

T. hirsutissima L. Sp. PI. 1: 140. 1753. Sc. USA, MEX (Cam, Chis, Col, Dgo, Gro, Hgo, Jal, Mich, Nay, Oax, Pue, QR, SLP, Sin, Tab, Tam, Ver), ANT, MA (Bel, CR, ES, Gua, Hon, Nic, Pan), SA. HMF, SV, TDF, TEF, TSEF. 0-2,600 m.

T. maculata Jacq. Enum. Syst. PI. 14. 1760. Li. MEX (Cam, Chis, Jal, Oax, QR, SLP, Sin, Ver), ANT, MA (Bel, CR, ES, Gua, Hon, Nic, Pan), SA. TEF. 200-1,400 m.

T. pedicellata D. Nash. FI. Veracruz 18. 1981. Li, Sc. MEX (Pue, Qro, Ver). TEF. 0-350 m.

T. umbellata Kunth. Nov. Gen. Sp. (quarto ed.) 3: 79, t. 202. 1818. Sc. MEX (Cam, Chis, Gro, Oax, Tab, Yuc), MA (Bel, Gua, Hon, Nic). SV, TDF, TEF, TSEF. 20-480 m.

T. volubilis L. Sp. PI. 1: 140. 1753. Sc. USA, MEX (BCS, Cam, Chis, Col, Gro, Hgo, Jal, Mex, Mich, Oax, Pue, QR, SLP, Sin, Son, Tam, Ver, Yuc), ANT, MA (Bel, CR, ES, Gua, Hon, Nic, Pan), SA. SV, TDF. 0-1,800 m.

\section{Cactaceae (2/9)}

Epiphyllum anguliger (Lem.) G. Don. Encycl. PI. (new ed., 1855) 1380. 1855. He. MEX (Gro, Hgo, Jal, Mex, Mich, Nay, Oax). QF, TSEF. 100-2,200 m.

E. pumilum (Vaupel) Britton \& Rose. Contr. U.S. Natl. Herb. 16: 258 (1913). He. MEX (Chis, Oax, Tab, Ver), MA (Gua). HMF, SV, TEF, TSEF. 100-1,300 m.

Selenicereus coniflorus (Weing.) Britton \& Rose. Contr. U.S. Natl. Herb. 12(10): 430. 1909. USA, MEX (Chis, Oax, Tab, Ver). XS. $250 \mathrm{~m}$.

S. donkelaarii (Salm-Dyck) Britton \& Rose. Cactaceae 2: 200, f. 276. 1920. Li. MEX (Cam, QR, Tab, Yuc), MA (Bel). MG, SAV, SV, TDF, TSEF. 0-100 m.

S. hamatus (Scheidw.) Britton \& Rose. Contr. U.S. Natl. Herb. 12(10): 430. 1909. Li. MEX (Oax, Ver). CF. NA.

S. hondurensis (K.Schum.) Britton \& Rose. Contr. U.S. Natl. Herb. 12(10): 430. 1909. Li. MEX (Chis), MA (Gua, Hon). TEF. NA.

S. murrillii Britton \& Rose. Cactaceae 2: 206-207, f. 285c-d. 1920. Li. MEX (Col, Jal). NA. 
Appendix 2. Continuation.

S. pteranthus (Link \& Otto) Britton \& Rose. Contr. U.S. Natl. Herb. 12(10): 431. 1909. He. MEX (Cam, Chis, Tab, Ver), ANT, MA (Pan). TEF, TSEF. 0-500 m.

S. tricae D.R. Hunt. Bradleya 7: 91, 89-90, f. 1A-C. 1989. Li. MEX (Chis), MA (Bel). TEF. NA.

\section{Cannabaceae (1/1)}

Celtis iguanaea (Jacq.) Sarg. Silva 7: 64. 1895. Li, Sc. USA, MEX (Cam, Chis, Chih, Gto, Gro, Hgo, Jal, Mich, Mor, Oax, Pue, Qro, QR, SLP, Sin, Son, Tab, Tam, Ver, Yuc), ANT, MA (Bel, CR, ES, Gua, Hon, Nic, Pan), SA. HMF, RF, TDF, TEF, TSEF. 0-1,500 m.

\section{Capparaceae (1/1)}

Cynophalla flexuosa (L.) J. Presl. Prir. Rostlin 2: 275. 1825. Sc. USA, MEX (Cam, Chis, Col, Gro, Jal, Mich, Nay, Oax, QR, Sin, Son, Tam, Yuc), ANT, MA (CR, ES, Nic, Pan), SA. TDF. 0-250 m.

\section{Caprifoliaceae $(2 / 2)$}

Lonicera pilosa (Kunth) Spreng. Nov. Gen. Sp. (quarto ed.) 3: 427. 1818. Sc. MEX (Ags, Chis, Chih, Coa, DF, Dgo, Gto, Hgo, Jal, Mex, Mich, Mor, Nay, NL, Oax, Qro, SLP, Sin, Son, Tam, Zac). CF, HMF, PQF, QF. 1,400-3,000 m.

Valeriana subincisa Benth. PI. Hartw. 39. 1840. Li. MEX (Chis, Coa, Hgo, NL, Oax, Pue, Qro, SLP, Tam, Ver), MA (Gua). CF, HMF, QF. 1,250-2,800 m.

\section{Celastraceae $(\mathbf{8} / 12)$}

Celastrus pringlei Rose. Contr. U.S. Natl. Herb. 5(4): 195. 1899. Li. MEX (Chis, Col, Dgo, Gro, Jal, Mex, Mich, Mor, Oax, SLP, Ver). HMF, QF. 2,000-2,600 m.

C. vulcanicolus Donn. Sm. Bot. Gaz. 61(5): 373. 1916. Li, Sc. MEX (Chis, Oax, Ver), MA (Gua, Hon, Nic). HMF, TEF. 620$2,800 \mathrm{~m}$.

Cheiloclinium belizense (Standl.) A.C. Sm. Brittonia 3(3): 540. 1940. Li, Sc. MEX (Chis), ANT, MA (Bel, CR, Gua, Hon, Nic, Pan), SA. NA.

Cuervea kappleriana (Miq.) A.C. Sm. Brittonia 3(3): 399-401. 1940. Li, Sc. MEX (Tab, Ver), MA (CR, Hon, Pan), SA. TEF, TSEF. 110-150 m.

Elachyptera floribunda (Benth.) A.C. Sm. Bot. Voy. Sulphur 78. 1844. Li, Sc. MEX (Chis, QR, Ver), MA (Gua, Hon, Pan), SA. MG, RF. 8-100 m.

Hippocratea volubilis L. Sp. PI. 2: 1191. 1753. Li, Sc. USA, MEX (Cam, Chis, Gro, Jal, Mex, Mor, Oax, Sin, Tab, Tam, Ver), ANT, MA (Bel, CR, Gua, Hon, Nic, Pan), SA. HMF, MG, RF, SV, TDF, TEF. 0-1,000 m.

Pristimera caribaea (Urb.) A.C. Sm. Brittonia 3(3): 378-380. 1940. Li. MEX (Ver), ANT. TEF. 160-400 m.

P. celastroides (Kunth) A.C. Sm. Brittonia 3(3): 371. 1940. Li, Sc. MEX (Cam, Chis, Col, Dgo, Gto, Gro, Jal, Mex, Mich, Mor, Nay, Oax, Pue, Qro, QR, SLP, Sin, Tab, Tam, Ver, Yuc, Zac), MA (Bel, ES, Gua, Hon, Nic), SA. HMF, RF, TDF, TEF, TSEF. 0-1,300 m.

Salacia cordata (Miers) Mennega. Novon 2(3): 232. 1992. Li, Sc. MEX (Chis, Oax, Tab, Ver), MA (CR, Nic, Pan), SA. HMF, TEF. 100-1,750 m.

S. impressifolia (Miers) A.C. Sm. Bull. Torrey Bot. Club 66(4): 247. 1939. Li, Sc. MEX (Oax, Tab, Ver), MA (Hon, Nic, Pan), SA. TEF. 0-75 m.

Semialarium mexicanum (Miers) Mennega. Proc. Kon. Ned. Akad. Wetensch. C 91(3): 316. 1988. Li, Sc. MEX (Cam, Chis, Dgo, Gro, Jal, Mex, Mich, Mor, Oax, QR, Tab, Yuc), MA (Nic, Pan), SA. RF, TDF, TEF, TSEF, XS. 0-800 m.

\section{Combretaceae $(1 / 7)$}

Combretum argenteum Bertol. Novi Comment. Acad. Sci. Inst. Bononiensis 4: 412. 1840. Li, Sc. MEX (Cam, Chis, Gro, Oax, Ver, Yuc), MA (ES, Gua, Hon, Nic), SA. SV, TDF, TSEF. 0-1,300 m.

C. decandrum Jacq. Enum. Syst. PI. 19. 1760. Li, Sc. MEX (Chis, Gro, Jal, Nay, Oax, Tab), ANT, MA (CR, ES, Gua, Nic, Pan), SA. RF, SV, TDF, TSEF. 0-1,300 m.

C. farinosum Kunth. Nov. Gen. Sp. (folio ed.) 6: 87. 1823. Li, Sc. MEX (Cam, Chis, Col, Dgo, Gro, Jal, Mex, Mich, Mor, Nay, Oax, Pue, SLP, Sin, Tab, Tam, Ver, Yuc), MA (CR, ES, Gua, Hon, Nic), SA. RF, TDF, TF. 0-2,000 m.

C. fruticosum (Loefl.) Stuntz. U.S.D.A. Bur. PI. Industr. Invent. Seeds 31: 86-87. 1914. Li, Sc. USA, MEX (Cam, Chis, Chih, Col, Gro, Hgo, Jal, Mex, Mich, Mor, Nay, Oax, Pue, Sin, Tab, Ver, Yuc), ANT, MA (Bel, CR, ES, Gua, Hon, Nic, Pan), SA. RF, SV, TDF, TEF, TSEF. 0-1,500 m.

C. igneiflorum Rendón \& R. Delgad. Novon 21(4): 483-485, f. 1. 2011. Li, Sc. MEX (Col, Jal, Oax). TDF. 0-360 m.

C. laxum Jacq. Enum. Syst. Pl. 19. 1760. Li, Sc. MEX (Cam, Chis, Col, Gro, Jal, Mich, Mor, Nay, Oax, QR, Tab, Ver), ANT, MA (Bel, CR, Gua, Hon, Nic, Pan), SA. RF, TDF, TEF, TSEF, MG. 0-1,000 m.

C. rovirosae Exell. J. Linn. Soc. Bot. 55(356): 134-135, f. 5e. 1953. Li, Sc. MEX (Cam, Chis, Oax, Tab, Ver), MA (Bel, Gua, Hon, Nic, Pan). RF, SV, TDF. 0-800 m. 
Appendix 2. Continuation.

\section{Connaraceae $(3 / 8)$}

Cnestidium rufescens Planch. Linnaea 23: 440. 1,850. Li, Sc. MEX (Cam, Chis, Oax, QR, Tab, Ver), ANT, MA (Bel, CR, Gua, Hon, Nic, Pan), SA. RF, SV, TDF, TEF. 0-1,100 m.

Connarus lentiginosus Brandegee. Univ. Calif. Publ. Bot. 6(8): 186. 1915. Li, Sc. MEX (Chis), MA (CR, Gua, Nic, Pan). TEF. 0$1,800 \mathrm{~m}$.

C. schultesii Standl. Bot. Mus. Leafl. 9: 173. 1941. Li. MEX (Chis, Oax, Ver). SAV, TEF, TSEF. 100-700 m.

C. stenophyllus Standl. \& L.O.Williams ex A.Molina. Li, Sc. MEX (Chis, Gro, Oax). HMF, PQF, SV. 400-1,300 m.

Rourea glabra Kunth. Nova Genera \& Species Plantarum (quarto ed.) 7: 41-42. 1824. Li, Sc. MEX (Cam, Chis, Col, Gro, Jal, Mich, Nay, Oax, Pue, Qro, QR, SLP, Sin, Tab, Tam, Ver, Yuc), ANT, MA (Bel, CR, ES, Gua, Hon, Nic, Pan), SA. HMF, QF, RF, SV, TDF, TEF, TSEF. 0-800 m.

R. schippii Standl. Publ. Carnegie Inst. Wash. 461:58. 1935. Li, Sc. MEX (Chis, Tab, Ver), MA (Bel, CR, Gua, Hon). TEF. 0-1,400 m.

R. suerrensis Donn. Sm. Bot. Gaz. 23(1): 5-6. 1897. Li, Sc. MEX (Tab), MA (CR, Hon, Nic, Pan). TEF. NA.

R. vulcanicola Forero. Caldasia 29(1): 19. 2007. Li. MEX (Ver). QF, TEF. 1,300-1,400 m.

\section{Convolvulaceae $(12 / 67)$}

Bonamia mexicana J.A. McDonald. Biótica 12(3): 221-224, f. 3. 1987. Li, Sc. MEX (Jal), MA (CR, ES). TDF, TSEF. 300 m.

B. sulphurea (Brandegee) Myint \& D.B. Ward. Phytologia 17(3): 178. 1968. Li, Sc. MEX (Cam, Chis, Gro, Jal, Ver), MA (Gua, Hon, Nic, Pan), SA. SV, TDF. 300-675 m.

Calycobolus nutans (Moc. \& Sessé ex Choisy) D.F. Austin. Ann. Missouri Bot. Gard. 58(2): 244. 1971 [1972]. Li, Sc. MEX (Col, Gro, Mex, Mich, Mor, Oax, Pue). SV, TDF, TF. 150-1,650 m.

Convolvulus nodiflorus Desr. Encycl. 3: 557. 1789[1792]. Li. MEX (Cam, Chis, Gro, Jal, Mich, Oax, QR, SLP, Tam, Ver, Yuc), ANT, MA (Bel, CR, ES, Gua, Hon, Nic), SA. QF, SV, TDF, TEF, TSEF. 0-1,500 m.

Ipomoea ampullacea Fernald. Proc. Amer. Acad. Arts 33(5): 89. 1897. Li. MEX (Col, Gro, Jal, Mex, Mich, Sin). TDF, TSEF. 150$1,350 \mathrm{~m}$.

I. anisomeres B.L. Rob. \& Bartlett. Proc. Amer. Acad. Arts 43(2): 57. 1907. Li. MEX (Cam, Chis, QR, Tab, Ver, Yuc), MA (Bel, CR, Gua, Hon, Nic, Pan), SA. TDF, TEF, TSEF. 0-500 m.

I. aurantiaca L.O. Williams. Fieldiana, Bot. 32(12): 187. 1970. Li. MEX (Chis), MA (CR, Gua, Nic). SV, TDF. 500-1,000 m.

I. batatoides Choisy. Mém. Soc. Phys. Genève 8(1): 58-59. 1838[1837]. Li. MEX (Chis, Jal, Oax, Qro, Ver), MA (Bel, CR, ES, Hon, Nic, Pan), SA. HMF, QF, SV, TEF, TSEF. 0-2,000 m.

I. bernoulliana Peter. Nat. Pflanzenfam. IV(3a): 30. 1897[1891]. Li. MEX (Chis), MA (CR, Gua, Hon, Nic). TDF. 300-1,400 m.

I. bombycina (Choisy) Benth. \& Hook. f. Gen. PI. 2: 873. 1876. Li. MEX (Gro, Jal). TDF. 0-300 m.

I. bracteata Cav. Icon. 5: 51, t. 477. 1799. Li. MEX (BCS, Chih, Col, Dgo, Gto, Gro, Jal, Mex, Mich, Mor, Nay, Oax, Pue, Sin, Son, Ver). QF, TDF, TF, TSEF. 0-1,700 m.

I. carnea Jacq. subsp. carnea. Enum. Syst. PI. 13. 1760. Li, Sc. MEX (Cam, Chis, QR, Tab, Ver, Yuc), MA (Bel, Nic), SA. MG, XS. 0-800 m.

I. conzattii Greenm. Publ. Field Columb. Mus. Bot. Ser. 2(6): 258. 1907. Li. MEX (Mor, Oax, Ver). TDF, XS. 1,500-2,170 m.

I. crinicalyx S. Moore. Trans. Linn. Soc. London, Bot. 4(3): 402, t. 27, f. 1. 1895. Li. MEX (Cam, Chis, Mich, QR, Yuc), MA (Bel, Gua, Nic), SA. SV, TDF, TSEF. 0-1,000 m.

I. cuprinacoma E. Carranza \& J.A. McDonald. Lundellia 4: 151-156. 2004. Li. MEX (Gro, Jal, Mich). TDF. 1,000-2,000 m.

I. dumosa (Benth.) L.O. Williams. Fieldiana, Bot. 32(12): 190. 1970. Li. MEX (Chis, Dgo, Gro, Hgo, Jal, Mex, Mich, Mor, Oax, Qro, SLP, Sin, Ver), MA (CR, ES, Gua, Hon, Nic, Pan). HMF, PQF, QF, TDF, TEF. 1,800-2,300 m.

I. elongata Choisy. Prodr. 9: 355. 1845. Li. MEX (Gro, Mex, Mich, Mor, Oax, Pue, Qro), MA (Gua). PQF, QF, TDF. 1,500-2,000 m.

I. fissifolia (McPherson) Eckenw. Brittonia 41(1): 79. 1989. Li. MEX (Mich). 1400-1450 m.

I. funis Schltdl. \& Cham. var langlassei (House) O’Donell. Lilloa 29: 41. 1959. Li. MEX (Gto, Gro, Mex, Mich, Pue, Ver). CF, PQF, QF. 2,000-2,300 m.

I. jalapa (L.) Pursh. Fl. Amer. Sept. 146-147. 1814. Li. MEX (Cam, Gto, Hgo, Jal, Qro, QR, SLP, Tam, Ver, Yuc), ANT, MA (Bel, CR, Gua, Hon, Nic), SA. QF, TDF, TSEF. 24-1,350 m.

I. leucotricha Donn. Sm. Bot. Gaz. 23(1): 10-11. 1897. Li. MEX (Chis), MA (CR, Gua, Nic). HMF, TEF. NA.

I. lindenii M. Martens \& Galeotti. Bull. Acad. Roy. Sci. Bruxelles 12(2): 264. 1845. Li. MEX (Cam, Chis, Gro, Jal, Mex, Mich, Oax, Pue, Qro, Ver), MA (Bel, CR, ES, Gua, Hon, Nic, Pan), SA. HMF, RF, TDF, TF, TSEF. 0-1,600 m.

I. lottiae J.A. McDonald. Biótica 12(3): 219-221, f. 2. 1987. Li. MEX (Gro, Jal, Mich). TDF, TSEF. 100-300 m.

I. microsepala Benth. Bot. Voy. Sulphur 136. 1844[1845]. Li. MEX (Col, Dgo, Gro, Jal, Mex, Mich, Nay, Oax, Sin, Ver), MA (Gua). CF, QF, SV, TDF, TEF. 10-1,600 m.

I. neei (Spreng.) O’Donell. Lilloa 29: 69. 1959. Li. MEX (Chis, Col, Gro, Jal, Mex, Mich, Nay, Oax, Sin, Tab, Ver), MA (CR, ES, Gua, Hon, Nic, Pan). HMF, QF, TDF, TEF, TSEF. 140-1,500 m. 
Appendix 2. Continuation.

I. nil (L.) Roth. Catal. Bot. 1: 36. 1797. Li. MEX (Chis, Gro, Jal, Mich, Oax, SLP, Tam, Ver), MA (Bel, CR, ES, Gua, Hon, Nic, Pan), SA. SV, TDF, TEF, TSEF. 0-600 m.

I. orizabensis (G. Pelletan) Ledeb. ex Steud. var. novogaliciana J.A. McDonald. Nomencl. Bot. (ed. 2) 818. 1841. Li. MEX (Mex, Mich). PQF. 1,700-2,150 m.

I. orizabensis G. Pelletan) Ledeb. ex Steud. var. orizabensis. Nomencl. Bot. (ed. 2) 818. 1841. Li. MEX (Chis, Chih, Coa, DF, Dgo, Gto, Hgo, Jal, Mex, Mich, Mor, Nay, NL, Oax, Pue, Qro, SLP, Sin, Tab, Tam, Tla, Ver, Zac), MA (Gua). CF, HMF, PQF, QF, SV, TDF, TEF. 850-2,750 m.

I. pedicellaris Benth. Bot. Voy. Sulphur 135. 1845. Li. MEX (Chis, Chih, Col, Dgo, Gro, Hgo, Jal, Mich, Mor, Nay, Oax, Pue, Qro, SLP, Sin, Son, Tab, Tam, Ver), MA (ES, Gua, Hon). QF, RF, TDF, TF, TSEF, XS. 10-1,300 m.

I. philomega (Vell.) House. Ann. New York Acad. Sci. 18(6): 246. 1908. Li. MEX (Hgo, Oax, Tab, Ver), ANT, MA (Bel, CR, Gua, Hon, Nic, Pan), SA. HMF, RF, SV, TEF. 100-150 m.

I. populina House. Ann. New York Acad. Sci. 18(6): 226. 1908. Li. MEX (Chis, Gro, Oax), MA (Gua, Hon, Nic). SV, TDF, TSEF. 15-1,200 m.

I. praecana House. Ann. New York Acad. Sci. 18: 227. 1908. Li. MEX (Chis, Col, Gro, Mex, Mich, Mor, Oax, QR), MA (Gua, Hon, Nic). QF, TDF. 150-1300 m.

I. pruinosa McPherson. Contr. Univ. Michigan Herb. 14: 88. 1980. Li. MEX (Gro, Mich). TDF. 400-1,200 m.

I. pseudoracemosa McPherson. Contr. Univ. Michigan Herb. 14: 88. 1980. Li. MEX (Jal, Mich, Nay). TDF. 900-1,400 m.

I. reticulata O'Donell. Lilloa 26: 389-391, t. 15. 1953. Li. MEX (Chis, Qro, Tam, Ver), MA (CR, Gua, Hon), SA. HMF, QF, TDF, TEF. 150-1,200 m.

I. robinsonii House. Ann. New York Acad. Sci. 18(6): 257. 1908. Li. MEX (Oax). TDF, XS. 830-1,150 m.

I. sagittata Poir. Voy. Barbarie 2: 122-123. 1789. Li. USA, MEX (Cam, Chis, QR, SLP, Tab, Tam, Ver, Yuc), ANT, MA (Bel, Gua). SV, TDF. 5-300 m.

I. santillanii O'Donell. Anales Inst. Biol. Univ. Nac. México 12: 93. 1941. Li. MEX (Chis, Col, Gro, Mex, Mich, Oax, Ver), MA (CR, ES, Gua, Nic). CF, PQF, SV, TDF, TEF, TSEF. 200-2,200 m.

I. seducta House. Ann. New York Acad. Sci. 18(6): 241. 1908. Li. MEX (Chis, Gro, Jal, Mich, Oax, Sin, Tab), MA (Gua). HMF, QF, TDF, TEF. 600-1,800 m.

I. sepacuitensis Donn. Sm. Bot. Gaz. 56(1): 59. 1913. Li. MEX (Chis, QR), MA (Bel, Gua). HMF, TEF. 0-125 m.

I. spectata J.A. McDonald. Harvard Pap. Bot. 4: 49. 1993. Li. MEX (Jal, Mich). PQF. 1,100-1,600 m.

I. squamosa Choisy. Prodr. 9: 376-377. 1845. Li, Sc. MEX (Cam, Chis, Gro, Mor, Pue, Tam, Ver, Yuc), MA (Bel, CR, ES, Gua, Hon, Nic, Pan), SA. TDF. 0-300 m.

I. steerei (Standl.) L.O. Williams. Fieldiana, Bot. 32(12): 195, f. 2. 1970. Li. MEX (Cam, QR, Yuc), MA (Gua, Hon). SV, TDF, TSEF. 0-500 m.

I. suaveolens (M. Martens \& Galeotti) Hemsl. Biol. Cent.-Amer. Bot. 2(11): 394. 1882. Li. MEX (Chis, Gto, Gro, Hgo, Mex, Mich, Nay, Oax, Qro, Sin, Ver), MA (CR, ES, Gua). QF, TDF, XS. 0-15,00 m.

I. suffulta (Kunth) G. Don. Gen. Hist. 4: 276. 1838. Li. MEX (Chis, Gro, Jal, Mex, Mich, Nay, Oax), MA (Gua). PQF, SAV, TDF. 1,000-1,800 m.

I. tiliacea (Willd.) Choisy. Prodr. 9: 375. 1845. Li. MEX (Cam, Chis, Gro, Mich, Oax, QR, Tab, Tam, Ver), ANT, MA (Bel, CR, ES, Gua, Hon, Nic, Pan), SA. SV, TDF, TEF, TSEF. 0-550 m.

I. urbinei House. Muhlenbergia 3(3): 41, t. 2, f. b. 1907. Li. MEX (Col, Gro, Mich, Pue). QF, TDF. 1,200-1,700 m.

I. violacea L. Sp. PI. 1: 161. 1753. Li. USA, MEX (Cam, Dgo, Jal, QR, Yuc), ANT, MA (Bel, Pan), SA, AS. TDF. 0-1,000 m.

Iseia luxurians (Moric.) O’Donell. Bol. Soc. Argent. Bot. 5(1-2): 77. 1953. Li. MEX (Yuc), ANT, MA (CR, Hon, Nic, Pan), SA. TEF. NA.

Itzaea sericea (Standl.) Standl. \& Steyerm. Publ. Field Mus. Nat. Hist. Bot. Ser. 23(2): 83. 1944. Li, Sc. MEX (Cam, Chis, Oax, QR, Tab, Ver), MA (Bel, CR, Gua, Hon, Nic). SV, TEF, TSEF. 100-150 m.

Jacquemontia abutiloides Benth. Bot. Voy. Sulphur 34-35. 1844. Li, Sc. MEX (BC, BCS, Son). RF, TDF, XS. 0-490 m.

J.confusa Meisn. Fl. Bras. 7: 294, t. 106, f. 2. 1869. Li. MEX (Cam, Chis, Oax), MA (Bel, Hon, Nic), SA. SV, TSEF. $170-250$ m.

J. havanensis (Jacq.) Urb. Symb. Antill. 3: 342. 1902. Li. USA, MEX (QR, Yuc), ANT, MA (Bel). TDF, TSEF. 5 m.

J. mexicana (Loes.) Standl. \& Steyerm. Publ. Field Mus. Nat. Hist. Bot. Ser. 23(2): 84. 1944. Li. MEX (Chis, Oax), MA (CR, ES, Gua, Nic). SV, TDF. 95-260 m.

J. oaxacana (Meisn.) Hallier f. Bot. Jahrb. Syst. 16(4-5): 543. 1893. Li. MEX (BCS, Cam, Oax, SLP, Tam, Ver). SV, TDF, TSEF. 10$500 \mathrm{~m}$.

J. pentanthos (Jacq.) G. Don. Gen. Hist. 4: 283. 1838. Li. USA, MEX (Cam, Chis, Chih, Col, Dgo, Gto, Gro, Jal, Mich, NL, Oax, Pue, Qro, QR, SLP, Sin, Tam, Ver, Yuc), ANT, MA (Bel, CR, ES, Gua, Hon, Nic, Pan), SA. HMF, SAV, SV, TDF, TSEF. 0-1,500 m.

Maripa nicaraguensis Hemsl. Biol. Cent.-Amer. Bot. 2(11): 382. 1882. Li. MEX (Chis), MA (Bel, CR, Gua, Hon, Nic, Pan), SA. TEF. 120-200 m.

Merremia aurea (Kellogg) O'Donell. Lilloa 6: 494. 1941. Li. MEX (BCS). TDF. 0-370 m. 
Appendix 2. Continuation.

M. cielensis J.A. McDonald. Syst. Bot. 33: 552. 2008. Li. MEX (Tam). HMF, TDF. 900 m.

M. discoidesperma (Donn. Sm.) O’Donell. Lilloa 6(2): 495-496. 1941. Li. MEX (Chis, Ver), ANT, MA (CR, Gua, Nic, Pan). HMF, SV, TEF. 0-200 m.

M. dissecta (Jacq.) Hallier f. Bot. Jahrb. Syst. 16(4-5): 552. 1893. Li. USA, MEX (BC, BCS, Cam, Chis, Coa, Col, Gro, Hgo, Jal, Mex, Mich, Mor, Nay, NL, Oax, QR, SLP, Sin, Son, Tab, Tam, Ver, Yuc), ANT, MA (Bel, CR, ES, Gua, Hon, Nic, Pan), SA. RF, SV, TDF, TEF, TF, TSEF. 0-1,300 m.

M. palmeri (Hallier) Hallier f. Jahrb. Hamburg. Wiss. Anst. Beih. 3: 38. 1899. Li. MEX (Gro, Jal, Mich, Oax, Sin, Son). TDF, TSEF. 14-400 m.

M. tuberosa (L.) Rendle. Fl. Trop. Afr. 4(2): 104. 1906[1905]. Li. USA, MEX (Cam, Chis, Gro, Hgo, Oax, QR, Tab, Ver, Yuc), ANT, MA (Bel, CR, ES, Gua, Hon, Nic, Pan), SA. RF, SV, TDF, TEF, TSEF. 10-400 m.

M. umbellata (L.) Hallier f. Bot. Jahrb. Syst. 16(4-5): 552. 1893. Li. USA, MEX (Cam, Chis, Col, Gro, Jal, Mex, Mich, Mor, Nay, Oax, Pue, Qro, QR, SLP, Sin, Tab, Tam, Ver, Yuc), ANT, MA (Bel, CR, ES, Gua, Hon, Nic, Pan), SA. SV, TDF, TEF, TSEF. 7$1,200 \mathrm{~m}$.

Odonellia hirtiflora (M. Martens \& Galeotti) K.R. Robertson. Brittonia 34(4): 418-421. 1982. Li. MEX (Chis, Oax, Tab, Ver), MA (Bel, CR, Gua, Nic, Pan), SA. SAV, SV, TEF. 50-150 m.

Operculina pinnatifida (Kunth) O’Donell. Lilloa 23: 432-435, t. 4-5. 1950. Li. USA, MEX (Cam, Chis, Coa, Col, Gro, Hgo, Jal, Mex, Mich, Mor, NL, Oax, Qro, QR, SLP, Sin, Son, Tab, Tam, Ver, Yuc), ANT, MA (Gua). SV, TF, RF, TDF, TSEF. 10-1,200 m.

O. pteripes (G. Don) O'Donell. Lilloa 23: 435-437, t. 6. 1950. Li. MEX (Cam, Chis, Chih, Col, DF, Gro, Jal, Mex, Mich, Mor, Nay, Oax, Sin, Son), MA (CR, ES, Gua, Hon, Nic, Pan), SA. MG, QF, SV, TDF, TF. 240-1,250 m.

Turbina corymbosa (L.) Raf. Fl. Tellur. 4: 81. 1836 [1838]. Li, Sc. USA, MEX (Cam, Chis, Gro, Hgo, Jal, Mex, Mich, Mor, Nay, Oax, QR, SLP, Sin, Tab, Tam, Ver, Yuc), ANT, MA (Bel, CR, ES, Gua, Hon, Nic, Pan), SA. QF, SV, TDF, TEF, TSEF. 0-1,300 m.

\section{Cucurbitaceae (9/12)}

Cionosicys macranthus (Pittier) C. Jeffrey. Kew Bull. 25(2): 200-201. 1971. Li. MEX (Cam, Chis, Mor, Oax, Pue, Tab, Ver), ANT, MA (Bel, CR, ES, Gua, Hon, Nic, Pan). SV, TEF. 0-150 m.

Cionosicys sp. Li. MEX (Ver), MA (Gua). TEF. 200-300 m.

Dieterlea fusiformis E.J. Lott. Brittonia 38(4): 407-410, f. 1. 1986. Li. MEX (Col, Jal, Mich, Sin, Son). TDF. 0-800 m.

D. maxima (Lira \& Kearns) McVaugh. Fl. Novo-Galiciana 3: 551. 2001. Li. MEX (Gro, Jal, Mich, Nay, Sin). SV, TDF. 150-1,850 m.

Doyerea emetocathartica Grosourdy. Méd. Bot. Criollo 1(2): 338-339. 1864. Li. MEX (Cam, Chis, Col, Gro, Jal, Nay, Oax, Pue, Qro, QR, SLP, Sin, Tam, Ver, Yuc), ANT, MA (Bel, CR, ES, Gua, Hon, Nic), SA. SV, TDF, TSEF. 0-1,000 m.

Gurania makoyana (Lem.) Cogn. Diagn. Cucurb. Nouv. 1: 17. 1876. Li. MEX (Chis, Tab), MA (Bel, CR, Gua, Hon, Nic, Pan), SA. SV, TEF. 270-1,500 m.

Hanburia mexicana Seem. Bonplandia (Hanover) 6(15): 293. 1858. Li. MEX (Hgo, Oax, Pue, Ver). HMF, RF, TSEF. 450-1,800 m.

Peponopsis adhaerens Naudin. Ann. Sci. Nat. Bot. sér. 4, 12: 89. 1860. Li. MEX (Hgo, Oax, Pue, Qro, SLP, Ver). HMF, PQF, TSEF. 50-1,600 m.

Psiguria triphylla (Miq.) C. Jeffrey. Kew Bull. 33(2): 353. 1978. Li. MEX (Chis, Dgo, Oax, QR, Tab, Ver), ANT, MA (Bel, CR, Gua, Hon, Nic, Pan), SA. RF, TEF, TSEF. 100-1,200 m.

P. warscewiczii (Hook. f.) Wunderlin. Phytologia 38(3): 219. 1978. Li. MEX (Chis), MA (Bel, CR, Gua, Hon, Nic, Pan), SA. RF, SV, TEF. 180-700 m.

Sechium edule (Jacq.) Sw. subsp. sylvestre Lira \& J. Castrejon. Acta Bot. Mex. 49: 57. 1999. Li. MEX (Oax, Pue, Qro, Ver). RF, TDF, TF. 1,100-1,200 m.

Sicydium schiedeanum Schltdl. Linnaea 7: 388. 1832. Li. MEX (Chis, Oax, Pue, Ver), MA (CR, ES, Gua, Nic), SA. SV, TDF, TEF. $100-2,000 \mathrm{~m}$.

\section{Dichapetalaceae (1/1)}

Dichapetalum donnell-smithii Engl. var. chiapasense (Standl.) Prance. Fl. Neotrop. 10: 28. 1972. Li, Sc. MEX (Chis, Qro, QR, Ver), MA (Bel, Gua, Hon, Pan). HMF, TEF. 0-1,400 m.

D. donnell-smithii Engl. var. donnell-smithii. Bot. Jahrb. Syst. 23: 144. 1896. Li, Sc. MEX (Chis, Jal, Oax, QR, Tab, Ver), MA (Bel, CR, ES, Gua, Hon, Pan), SA. HMF, TEF. 0-1,800 m.

\section{Dilleniaceae (3/5)}

Davilla kunthii A. St.-Hil. PI. Usuel. Bras. sub t. 22. 1824[1825]. Li, Sc. MEX (Cam, Chis, Oax, QR, Tab, Ver), ANT, MA (Bel, CR, Gua, Hon, Nic, Pan), SA. MG,QF, SAV, TEF. 0-500 m.

D. nitida (Vahl) Kubitzki. Mitt. Bot. Staatssamml. München 9: 95-99. 1971. Li, Sc. MEX (Chis, Ver), ANT, MA (Bel, CR, Gua, Hon, Nic, Pan), SA. SV, TEF, TSEF. 0-250 m. 
Appendix 2. Continuation.

Doliocarpus dentatus (Aubl.) Standl. J. Wash. Acad. 15(13): 286. 1925. Li, Sc. MEX (Cam, Chis, Oax, Tab, Ver), ANT, MA (Bel, CR, Gua, Hon, Nic, Pan), SA. MG, QF, RF, TEF, TSEF. 0-720 m.

Tetracera portobellensis Beurl. Kongl. Vetensk. Acad. Handl. 40: 113-114. 1854[1856]. Li. MEX (Chis, Gro, Jal, Mich, Nay), MA (CR, Nic, Pan), SA. RF, TEF. 50-310 m.

T. volubilis L. subsp. volubilis. Sp. PI. 1: 533. 1753. Li, Sc. MEX (Cam, Chis, Oax, QR, Tab, Ver, Yuc), ANT, MA (Bel, CR, ES, Gua, Hon, Nic, Pan), SA. MG, QF, SV, TDF, TEF. 0-900 m.

\section{Dioscoreaceae (1/1)}

Dioscorea mexicana Scheiew. Hort. Belge 4: 99, t. 76. 1837. Li. MEX (Chis, Col, Gro, Jal, Mex, Mich, Oax, Pue, SLP, Tab, Ver, Yuc), MA (Bel, CR, Gua, Hon, Nic, Pan). PQF, QF, TDF. 0-2,000 m.

\section{Euphorbiaceae (5/7)}

Bia cordata (Baill.) G.L.Webster. Contr. Univ. Michigan Herb. 25: 237. 2007. Li. MEX (Oax, Tab, Ver), MA (CR, Nic). TEF. 100$300 \mathrm{~m}$.

Dalechampia scandens L. Sp. PI. 2: 1054. 1753. Li. MEX (BCS, Cam, Chis, Gro, Jal, Mich, Oax, QR, Tab, Ver, Zac), MA (Bel, CR, ES, Gua, Pan), SA. TDF, TSEF. 5-1,400 m.

Dalembertia populifolia Baill. Étude Euphorb. 346, pl. 5, f. 11-15. 1858. Li. MEX (Col, Gro, Jal, Mex, Mich, Nay, Oax, Son). QF, SV, TDF. 350-1,740 m.

Manihot chlorosticta Standl. \& Goldman. Contr. U.S. Natl. Herb. 13(10): 375. 1911. Li, Sc. MEX (Gro, Jal, Mich, Oax, Sin). TDF. 0-10 m.

Plukenetia carabiasiae J. Jiménez Ram. Anales Inst. Biol. Univ. Nac. Autón. México, Bot. 64(2): 55-58, f. 1. 1993. Li. MEX (Oax). HMF, TEF. $650 \mathrm{~m}$.

P. stipellata L.J. Gillespie. Syst. Bot. 18(4): 588, f. 3-6, 9. 1993. Li. MEX (Chis, Oax, Tab, Ver), MA (CR, Gua, Nic, Pan). HMF, SV, TEF. 150-1,300 m.

P. volubilis L. Sp. PI. 2: 1192. 1753. Li. MEX (Chis, Tab, Ver), MA (CR, Hon, Pan), SA. SV, TEF. 80-300 m.

\section{Fabaceae (26/94)}

Acacia gaumeri S.F. Blake. Proc. Biol. Soc. Wash. 34: 44. 1921. Sc. MEX (Cam, QR, Yuc), MA (Gua). MG, TDF. 200 m.

A. hayesii Benth. Trans. Linn. Soc. London 30(3): 524. 1875. Li, Sc. MEX (Cam, Chis, Gro, Oax, Tab, Ver), MA (CR, Gua, Hon, Pan), SA. TEF, TSEF. 0-200 m.

A. riparia Kunth var. riparia. Nov. Gen. Sp. (folio ed.) 6: 218. 1824. Sc. MEX (Chis, Col, Gro, Jal, Mich, Mor, Nay, Oax, Pue, QR, SLP, Sin, Ver, Yuc), ANT, MA (CR, ES, Pan), SA. RF, SV, TDF. 0-1,400 m.

A. riparia Kunth var. tucumanensis (Griseb.) Griseb. Abh. Königl. Ges. Wiss. Göttingen 24: 121. 1879. Sc. MEX (SLP), MA (ES, Gua), SA. SV, TDF, TEF, TSEF. 20-750 m.

A. tenuifolia (L.) Willd. Sp. Pl. Editio quarta 4(2): 1091. 1806. Li, Sc. MEX (Chis, Oax), MA (CR, ES, Hon, Pan), SA. TDF. 190$830 \mathrm{~m}$.

Adenopodia gymnantha Brenan. Kew Bull. 41(1): 87-90, f. 3. 1986. Li. MEX (Col, Gro, Jal, Mich, Nay). TDF, TSEF. 0-550 m.

A. oaxacana M. Sousa. Kew Bull. 41(1): 85-87, f. 2. 1986. Li, Sc. MEX (Oax). RF, TDF, TSEF. 20-190 m.

A. patens (Hook. \& Arn.) J.R. Dixon ex Brenan. Kew Bull. 41(1): 80. 1986. Li, Sc. MEX (Chis, Col, Gro, Jal, Mich, Nay, Oax), MA (ES, Gua, Hon, Nic). RF, TDF. 0-500 m.

Barbieria pinnata (Pers.) Baill. Hist. PI. 2: 263. 1870. Li. MEX (Chis, Oax), ANT, MA (Bel, CR, Gua, Pan), SA. TSEF. 150 m.

Bauhinia glabra Jacq. Enum. Syst. PI. 20. 1760. Li. MEX (Chis, Oax, Tab), ANT, MA (Bel, CR, Hon, Nic, Pan), SA. SV, TEF. 30$800 \mathrm{~m}$.

B. guianensis Aubl. Hist. Pl. Guiane 1: 377-378, pl. 145. 1775. Li. MEX (Oax, Tab), ANT, MA (Bel, CR, Hon, Nic, Pan), SA. TEF. NA.

B. herrerae (Britton \& Rose) Standl. \& Steyerm. Publ. Field Mus. Nat. Hist. Bot. Ser. 23(1): 10. 1943. Li, Sc. MEX (Cam, Chis, Mex, Mor, Nay, Oax, QR, Tab, Ver, Yuc), MA (Bel, CR, Gua, Hon, Nic). TEF, TSEF. 0-360 m.

B. microstachya (Raddi) J.F. Macbr. Contr. Gray Herb. 59: 22. 1919. Li. MEX (Chis), MA (Bel, Gua, Pan), SA. TEF, TSEF. 180$340 \mathrm{~m}$.

Caesalpinia globulorum Bakh. f. \& P. Royen. Blumea 12(1): 62. 1963. Li. MEX (Chis, Pue), ANT, SA. TEF. $160-600$ m.

Calopogonium caeruleum (Benth.) C. Wright. Anales Acad. Ci. Med. Habana 5: 337. 1868[1869]. Li. MEX (Chis, Col, Gro, Jal, Mex, Mich, Nay, Oax, SLP, Tab, Ver, Yuc), ANT, MA (Bel, CR, ES, Hon, Pan), SA. RF, SV, TDF, TEF, TSEF. 0-1,800 m.

Canavalia acuminata Rose. Contr. U.S. Natl. Herb. (9): 322. 1895. Li. MEX (Col, Gro, Jal, Mich, Nay, Oax, Sin). TDF, TSEF. 0$1,100 \mathrm{~m}$.

C. brasiliensis Mart. ex Benth. Comm. Legum. Gen. 71. 1837. Li. USA, MEX (Cam, Chis, Gro, Jal, Mich, Nay, Oax, QR, Sin, Tam, Ver, Yuc), ANT, MA (Bel, CR, ES, Gua, Hon, Nic, Pan), SA. RF, SAV, SV, TDF, TSEF. 50-1,000 m. 
C. dura J.D. Sauer. Brittonia 16(2): 129-130, f. 8. 1964. Li, Sc. MEX (Chis, Gro). CF, HMF. 1,500-2,000 m.

C. glabra (M. Martens \& Galeotti) J.D. Sauer. Brittonia 16(2): 130. 1964. Li. MEX (Chis, Mor, Oax, Pue, Tab, Ver), MA (Gua, Hon, Pan), SA. HMF, SV, TDF, TEF, TSEF. 200-1,500 m.

C. hirsutissima J.D. Sauer. Brittonia 16(2): 136, f. 11. 1964. Li. MEX (Chis, Col, Gro, Jal, Mex, Mich, Oax, Pue, Ver), MA (ES, Gua). HMF, QF. 750-2,000 m.

C. oxyphylla Standl. \& L.O. Williams. Ceiba 3(3): 201-202. 1953. Li. MEX (Chis, Oax, Tab, Ver), MA (Bel, CR, Gua, Hon, Nic, Pan), SA. SV, TEF. 100-2,000 m.

C. villosa Benth. Comm. Legum. Gen. 71. 1837. Li. MEX (Cam, Chis, Coa, Col, DF, Dgo, Gto, Gro, Hgo, Jal, Mex, Mich, Mor, Nay, NL, Oax, Pue, Qro, QR, SLP, Sin, Son, Tab, Tam, Ver, Zac), MA (Bel, CR, ES, Gua, Hon, Nic, Pan), SA. HMF, PQF, QF, RF, SV, TDF, XS. 450-2,200 m.

Centrosema macrocarpum Benth. Ann. Nat. Hist. 3: 436. 1839. Li, Sc. MEX (Oax, Tab), ANT, MA (Bel, CR, Hon, Nic, Pan), SA. CF, TEF. 100-900 m.

C. plumieri (Turpin ex Pers.) Benth. Comm. Legum. Gen. 54. 1837. Li. MEX (Cam, Chis, Col, Gro, Jal, Mex, Mich, Mor, Nay, Oax, QR, Tab, Ver, Yuc), ANT, MA (Bel, CR, ES, Gua, Hon, Nic, Pan), SA. HMF, PQF, SV, TDF, TSEF. 0-1,750 m.

C. pubescens Benth. Comm. Legum. Gen. 55. 1837. Li. MEX (Chis, Col, Gro, Hgo, Jal, Mex, Mich, Nay, Oax, QR, Tab, Ver, Yuc), ANT, MA (Bel, CR, ES, Gua, Hon, Nic, Pan), SA, AS. CF, HMF, RF, SV, TSEF. 100-1,300 m.

C. virginianum (L.) Benth. Comm. Legum. Gen. 56. 1837. Li. USA, MEX (Cam, Chis, Gro, Jal, Mor, Nay, NL, Oax, QR, SLP, Tab, Tam, Ver, Yuc), ANT, MA (Bel, CR, ES, Gua, Hon, Nic, Pan), SA. CF, QF, RF, SV, TDF, TEF. 10-30 m.

Clitoria mexicana Link. Enum. Hort. Berol. Alt. 2: 235. 1822. Li. MEX (Chis, Gro, Jal, Mex, Mich, Oax, Tam, Ver), MA (Gua, Hon, Nic). PQF, QF. 650-2,250 m.

Dalbergia brownei (Jacq.) Schinz. Bull. Herb. Boissier 6(9): 731. 1898. Sc. USA, MEX (Oax, Tab), ANT, MA (CR, ES, Hon, Nic, Pan), SA. MG, TDF, TEF. 25 m.

D. ecastaphyllum (L.) Taub. Nat. Pflanzenfam. 3(3): 335. 1894. Sc. USA, MEX (Tab), ANT, MA (Bel, CR, Gua, Hon, Nic, Pan), SA, AF. MG. 0-25 m.

D. glabra (Mill.) Standl. var. glabra. Publ. Field Mus. Nat. Hist. Bot. Ser. 8(1): 15. 1930. Sc. MEX (Cam, Chis, Oax, QR, Tab), MA (Bel, CR, ES, Gua, Hon). SAV, TEF, TSEF. 0-1,170 m.

Desmodium infractum DC. Prodr. 2: 330. 1825. Li. MEX (Chis, Gro, Jal, Mich, Nay, Oax, Tab, Ver), MA (Bel, CR, ES, Gua, Nic, Pan), SA. TDF, TSEF. 0-700 m.

D. macrodesmum (S.F. Blake) Standl. \& Steyerm. Fieldiana, Bot. 24(5): 230. 1946. Li. MEX (Chis, Oax, Tab, Ver), MA (Bel, Gua, Hon, Pan). HMF, SV, TDF, TEF. 220-1,100 m.

Dioclea guianensis Benth. Comm. Legum. Gen. 70. 1837. Li, Sc. MEX (Chis), ANT, MA (Bel, CR, Hon, Pan), SA. SV. $100-200$ m.

D. holtiana Pittier ex R.H. Maxwell. Ann. Missouri Bot. Gard. 77(3): 584-585. 1990. Li, Sc. MEX (Chis), MA (Bel, CR, Nic, Pan), SA. NA.

D. reflexa Hook. f. Niger FI. 306-307. 1849. Li. MEX (Chis, Tab), ANT, MA (Bel, CR, Gua, Hon, Nic, Pan), SA, AF. NA.

D. virgata (Rich.) Amshoff. Meded. Bot. Mus. Herb. Rijks Univ. Utrecht 52: 69. 1939. Li. MEX (Tab), MA (Bel, Gua, Hon, Nic, Pan), SA. QF. 40 m.

D. wilsonii Standl. Publ. Field Mus. Nat. Hist. Bot. Ser. 4(8): 310-311. 1929. Li, Sc. MEX (Chis, QR, Tab), ANT, MA (Bel, CR, Gua, Hon, Nic, Pan), SA. SV, TEF. 80-300 m.

Entada gigas (L.) Fawc. \& Rendle. Fl. Jamaica 4: 124. 1920. Li. MEX (Oax, Tab, Ver), ANT, MA (Bel, CR, Hon, Nic, Pan), SA, AF. RF, TEF. NA.

E. polystachya (L.) DC. Prodr. 2: 425. 1825. Li. MEX (Chis, Col, Gro, Jal, Mex, Mich, Nay, Oax, Sin, Tab), ANT, MA (Bel, CR, ES, Gua, Hon, Nic, Pan), SA. RF, SAV, TDF, TSEF. 0-550 m.

Galactia acapulcensis Rose. Contr. U.S. Natl. Herb. 5(3): 137. 1897. Li. MEX (BCS, Chis, Col, Gro, Jal, Mex, Mor, Nay, Oax, Sin), MA (Gua, Hon). TDF. 0-500 m.

G. brachystachys Benth. Comm. Legum. Gen. 63. 1837. Li. MEX (Coa, Dgo, Gto, Gro, Hgo, Jal, NL, Oax, Pue, Qro, SLP). HMF, PQF, TDF, XS. 750-2,000 m.

G. striata (Jacq.) Urb. Symb. Antill. 2(2): 320. 1900. Li. MEX (Cam, Chis, Chih, Jal, Mex, NL, Oax, QR, Tab, Ver, Yuc), ANT, MA (Bel, CR, ES, Gua, Hon, Nic, Pan), SA. PQF, SAV, SV, TDF, XS. 700-1,290 m.

G. viridiflora (Rose) Standl. Contr. U.S. Natl. Herb. 23(2): 502. 1922. Li, Sc. MEX (Col, Gro, Mex, Mich, Mor, Oax, Pue). TDF. 300-500 m.

Machaerium cirrhiferum Pittier. Contr. U.S. Natl. Herb. 20(12): 472. 1922. Li, Sc. MEX (Cam, Chis, Oax, QR, Tab, Ver), MA (Bel, CR, Hon, Nic, Pan), SA. TEF, TSEF. 120-360 m.

M. cobanense Donn. Sm. Bot. Gaz. 44(2): 108-109. 1907. Li, Sc. MEX (Chis, Oax, Ver), MA (CR, Gua, Nic), SA. HMF, PQF, TEF. $120-2,000 \mathrm{~m}$.

M. falciforme Rudd. Phytologia 24(2): 125. 1972. Li, Sc. MEX (Cam, Chis, Tab, Ver), MA (Bel, Gua, Hon, Nic). RF, TEF. 0-200 m. 
Appendix 2. Continuation.

M. floribundum Benth. var. floribundum. J. Proc. Linn. Soc. Bot. 4 (Suppl.): 68. 1860. Li, Sc. MEX (Chis, Oax, Tab, Ver), MA (Bel, CR, Gua, Hon, Pan), SA. QF, SV, TEF. 120-800 m.

M. isadelphum (E. Mey.) Amshoff. Meded. Bot. Mus. Herb. Rijks Univ. Utrecht 52: 53. 1939. Li, Sc. MEX (Chis, Mex, Oax, QR, Tab, Ver), ANT, MA (Bel, CR, ES, Hon, Nic, Pan), SA. QF, SAV, SV, TEF, TSEF. 0-475 m.

M. kegelii Meisn. Linnaea 21: 257-258. 1848. Li, Sc. MEX (Cam, Chis, Jal, Oax, Tab), MA (Bel, CR, ES, Hon, Nic, Pan), SA. RF, TEF, TSEF. 0-500 m.

M. Iunatum (L.) Ducke. Arch. Jard. Bot. Rio de Janeiro 4: 310. 1925. Sc. MEX (Tab), ANT, MA (Bel, CR, Nic, Pan), SA. NA.

M. salvadorense (Donn. Sm.) Rudd. Phytologia 22(1): 56. 1971. Li, Sc. MEX (Chis, Coa, Col, Gro, Jal, Mich, Nay, Oax, Ver), MA (Bel, CR, ES, Gua, Pan), SA. RF, TDF, TSEF. 20-850 m.

M. seemanii Benth. ex Seem. Bot. Voy. Herald 110. 1853. Li, Sc. MEX (Cam, Chis, QR, Tab), MA (Bel, CR, Gua, Hon, Nic, Pan), SA. PQF, TEF. 40-1,170 m.

Mimosa albida Humb. \& Bonpl. ex Willd. Sp. PI. 4(2): 1030. 1806. Li, Sc. MEX (Cam, Chis, Col, DF, Gto, Gro, Hgo, Jal, Mex, Mich, Mor, Nay, Oax, Pue, Qro, QR, Sin, SLP, Tab, Tam, Ver, Yuc, Zac), MA (Bel, CR, Gua, ES, Hon, Nic, Pan), SA. CF, PQF, QF, SV, TDF, TSEF. 10-2,170 m.

M. ervendbergii A. Gray. Proc. Amer. Acad. Arts 5: 178-179. 1862. Li. MEX (Chis, Jal, Oax, Pue, Tab, Ver), MA (Bel, CR, Gua, Nic). TDF, TEF, TSEF. 10-900 m.

M. hondurana Britton. N. Amer. Fl. 23(3): 169. 1928. Li. MEX (Chis), MA (Bel, ES, Gua, Hon, Nic). HMF, SV, TEF. 500-1,000 m. M. sinaloensis Britton \& Rose. N. Amer. Fl. 23(3): 163. 1928. Li. MEX (Sin). 0-200 m.

M. watsonii B.L. Rob. Proc. Amer. Acad. Arts 36(26): 473-474. 1901. Li. MEX (Chis, Gro, Oax, Tab, Ver), MA (Bel, CR, ES, Gua, Hon, Nic). PQF, QF, RF, SV, TDF, TEF. 30-1,250 m.

Mucuna argyrophylla Standl. Contr. U.S. Natl. Herb. 23(2): 504. 1922. Li. MEX (Chis, Oax, Pue, SLP, Tab, Ver), MA (Bel, ES, Gua, Hon, Nic). HMF, SV, TEF. 40-1,000 m.

M. holtonii (Kuntze) Moldenke. Phytologia 1(1): 7. 1933. Li. MEX (Chis), MA (Bel, CR, ES, Nic, Pan), SA. RF, SV, TEF. 30-1,160 m.

M. jarocha T.M. Moura, Mansano, Gereau \& A.M.G. Azevedo. Phytotaxa 89(1): 44-46, f. 1A-J. 2013. Li. MEX (Ver). 1,3001,550 m.

M. pruriens (L.) DC. var. pruriens. Prodr. 2: 405. 1825. Li. MEX (Cam, QR, Ver), AS. SV, TDF. NA.

M. sloanei Fawc. \& Rendle. J. Bot. 55(650): 36. 1917. Li. MEX (Chis, Gro, Jal, Mich, Oax, Tab, Ver, Yuc), ANT, MA (Bel, CR, ES, Gua, Hon, Nic, Pan), SA, AF. TEF, TSEF. 0-600 m.

Nissolia fruticosa Jacq. var. fruticosa. Enum. Syst. PI. 27. 1760. Li. MEX (Cam, Chis, Col, Gro, Jal, Mex, Mich, Mor, Nay, Oax, Pue, Qro, QR, SLP, Sin, Tam, Ver, Yuc), MA (Bel, CR, ES, Gua, Hon, Nic), SA. QF, RF, SV, TDF, TSEF, XS. 0-2,150 m.

N. gentryi Rudd. Acta Bot. Mex. 15: 26. 1991. Li. MEX (Sin, Son). TDF. 570-760 m.

N. laxior (B.L. Rob.) Rose. Contr. U.S. Natl. Herb.) 5(4): 162. 1899. Li. MEX (Col, Gro, Jal, Mex, Mich, Nay, Oax, Qro). QF, TDF, TSEF. 800-1,320 m.

N. leiogyne Sandwith. Bull. Misc. Inform. Kew 1937(5): 302-303. 1937. Li. MEX (Col, Gro, Jal, Mich, Sin). TDF, TSEF. 0-1,100 m.

N. microptera Poir. Encycl. Suppl. 4(1): 98-99. 1816. Li. MEX (Ags, Cam, Chis, Chih, Col, Dgo, Gto, Gro, Jal, Mex, Mich, Mor, Nay, Oax, Pue, Qro, Sin, Son, Zac). CF, QF, RF, SV, TDF, XS. 300-2,000 m.

N. platycarpa Benth. FI. Bras. 15(1A): 77. 1859. Li. MEX (Coa, Gro, Hgo, NL, Oax, Pue, Qro, SLP, Sin, Son, Tam, Ver). CF, QF, XS. 270-2,000 m.

N. pringlei Rose. Contr. U.S. Natl. Herb. 5(4): 159-160, f. 20. 1899. Li. MEX (Chih, Coa, Dgo, Gto, Gro, Jal, Mex, Mich, Mor, Oax, Pue, Qro, SLP, Sin, Zac). QF, XS. 1,500-2,320 m.

N. ruddiae R. Cruz \& M. Sousa. Acta Bot. Mex. 68: 66-70, f. 1. 2004. Li. MEX (Gro, Mor, Pue). TDF. 650-1,420 m.

Oxyrhynchus trinervius (Donn. Sm.) Rudd. Phytologia 15(5): 291. 1967. Li. MEX (Chis, Ver), MA (Bel, CR, Gua, Nic), SA. SAV, SV, TEF. 10-560 m.

Phaseolus albescens McVaugh ex R. Delgad. \& A. Delgado. Sida 18(3): 638-646, f. 1-5. 1999. Li. MEX (Jal, Mich). PQF. 1,640$1,788 \mathrm{~m}$.

P. Iunatus L. Sp. PI. 2: 724. 1753. Li. MEX (BCS, Cam, Chis, Col, DF, Gro, Jal, Mex, Mich, Mor, Nay, Oax, QR, Sin, Tab, Tam, Ver, Yuc), ANT, MA (Bel, CR, ES, Gua, Hon, Nic, Pan), SA, AF, AS. HMF, SV, TDF, TSEF. 0-1,578 m.

P. maculatus Scheele subsp. ritensis (M.E. Jones) Freytag. Sida Bot. Misc. 23: 257-260, f. 95, 97. 2002. Li. USA, MEX (Ags, Chih, Coa, Dgo, Gto, Hgo, Jal, Nay, Pue, Qro, SLP, Sin, Son, Tla, Zac). PQF, QF, TDF. 600-2,000 m.

P. pedicellatus Benth. Comm. Legum. Gen. 73. 1837. Li. MEX (BCS, Coa, Col, DF, Gto, Gro, Hgo, Jal, Mex, Mor, NL, Pue, Qro, SLP, Sin, Tam, Ver). CF, PQF, QF. 1780-2,700 m.

P. rotundatus Freytag \& Debouck. Sida Bot. Misc. 23: 132-134, f. 44, 46. 2002. Li. MEX (Jal, Oax). SV, TDF. 1,490-1,730 m.

Piptadenia flava (Spreng. ex DC.) Benth. Trans. Linn. Soc. London 30(3): 371. 1875. Sc. MEX (Chis, Col, Gro, Jal, Mich, Mor, Oax), ANT, MA (CR, ES, Gua, Hon, Pan), SA. SV, TDF, TSEF. 0-300 m.

Ramirezella calcoma Ochot.-Booth \& A. Delgado. Anales Inst. Biol. Univ. Nac. Autón. México, Bot. 65(1): 8-11, f. 1. 1994. Li. MEX (Mex). QF. 860-1,610 m. 
Appendix 2. Continuation.

R. crassa (McVaugh) Ochot.-Booth \& A. Delgado. Anales Inst. Biol. Univ. Nac. Autón. México, Bot. 65(1): 16. 1994. Li. MEX (Jal, Mich, Nay, Sin). QF, RF, TSEF. 200-1,500 m.

R. lozanii (Rose) Piper. Contr. U.S. Natl. Herb. 22(9): 669. 1926. Li. MEX (Col, Gro, Jal, Mich, Nay). HMF, PQF. 1,000-1,800 m.

R. strobilophora (B.L. Rob.) Rose var. buseri (Micheli) Maréchal, Mascherpa \& Stainier. Taxon 27(2-3): 199. 1978. Li. MEX (Chis, Chih, Col, Gro, Jal, Mex, Mich, Oax), MA (ES, Nic). HMF, QF, RF, TSEF. 0-1,800 m.

R. strobilophora (B.L. Rob.) Rose var. strobilophora. Contr. U.S. Natl. Herb. 8(1): 44. 1903. Li. MEX (Ags, Chis, Chih, Gro, Jal, Mich, Oax). RF, TDF. 1,300-1,800 m.

Rhynchosia discolor M. Martens \& Galeotti. Bull. Acad. Roy. Sci. Bruxelles 10(2): 199. 1843. Li. MEX (Chis, Chih, Dgo, Gro, Jal, Mex, Mich, Mor, Oax, Sin, Son, Zac), MA (Bel, ES, Gua). HMF, PQF, QF, TDF. 650-2,000 m.

R. erythrinoides Schltdl. \& Cham. Linnaea 5: 587. 1830. Li. MEX (Chis, Oax, Pue, Ver), ANT, MA (CR, Gua, Hon, Nic, Pan), SA. HMF, TEF. 250-980 m.

R. longeracemosa M. Martens \& Galeotti. Bull. Acad. Roy. Sci. Bruxelles 10(2): 198-199. 1843. Li. MEX (Cam, Chis, Hgo, Jal, NL, Oax, Pue, Qro, QR, SLP, Tab, Tam, Ver, Yuc), MA (Bel, CR, ES, Gua, Hon, Nic, Pan). SV, TDF, TSEF. 0-2,040 m.

R. macrocarpa Benth. PI. Hartw. 11. 1839. Li. MEX (Ags, Chih, Dgo, Gto, Hgo, Jal, Mich, SLP, Sin, Son, Zac). CF, PQF, QF, SV, XS. 1,750-2,500 m.

R. precatoria (Humb. \& Bonpl. ex Willd.) DC. Prodr. 2: 385. 1825. Li. MEX (BC, Chis, Chih, Col, Gro, Jal, Mex, Mich, Mor, Nay, Oax, SLP, Sin, Son, Ver), MA (CR, Nic), SA. SV, TDF, TSEF. 0-1,800 m.

R. reticulata (Sw.) DC. var. reticulata. Prodr. 2: 385. 1825. Li. MEX (Cam, Chis, Col, Gro, Jal, Mich, Nay, Oax, QR, SLP, Tab, Ver, Yuc), ANT, MA (CR, ES, Gua, Hon, Nic, Pan), SA. SV, TDF, TSEF. 90-1,000 m.

R. swartzii (Vail) Urb. Repert. Spec. Nov. Regni Veg. 15: 320. 1918. Li. USA, MEX (Cam, QR, Yuc), ANT, MA (Bel). TDF, TEF, TSEF. 11-270 m.

R. tarphantha Standl. Publ. Field Mus. Nat. Hist. Bot. Ser. 4(8): 214-215. 1929. Li. MEX (Gro, Jal, Oax). PQF, RF. 450-2,000 m.

R. yucatanensis Grear. Mem. New York Bot. Gard. 31(1): 103-105, f. 27. 1978. Li. MEX (Cam, QR, Yuc), MA (Gua). SV, TSEF. 128-750 m.

Senna peralteana (Kunth) H.S. Irwin \& Barneby. Mem. New York Bot. Gard. 35: 597. 1982. Li, Sc. MEX (Cam, Chis, Gro, Oax, QR, Ver, Yuc), MA (Bel, Gua). RF, SV, TEF, TSEF. 100-670 m.

S. quinquangualata (Rich.) H.S. Irwin \& Barneby var. quinquangulata. Mem. New York Bot. Gard. 35: 153. 1982. Li, Sc. MEX (Col, Dgo, Gro, Jal, Mich, Nay, Oax, Sin, Tab, Ver), ANT, MA (Bel, CR, Gua, Nic, Pan), SA. CF, PQF, QF, RF, SV, TDF. 0-1,200 m.

S. undulata (Benth.) H.S. Irwin \& Barneby. Mem. New York Bot. Gard. 35: 157. 1982. Sc. MEX (Cam, Chis, Nay, QR, Tab, Ver, Yuc), ANT, MA (Bel, CR, Gua, Hon, Nic, Pan), SA. HMF, TEF, TSEF. 0-980 m.

Sphinga platyloba (Bertero ex DC.) Barneby \& J.W. Grimes. Mem. New York Bot. Gard. 74(1): 164. 1996. Li, Sc. MEX (Cam, Chis, Jal, Oax, QR, Tab, Yuc), ANT, MA (Bel, CR, Gua, Nic), SA. TDF, TEF. 0-1,170 m.

Vigna speciosa (Kunth) Verdc. Kew Bull. 24(3): 552. 1970. Li. MEX (Chis, Gro, Jal, Mich, Nay, Oax, SLP, Sin, Son, Tab, Tam, Ver), MA (Bel, CR), SA. RF, SV, TDF. 0-1,400 m.

\section{Gelsemiaceae (1/1)}

Gelsemium sempervirens (L.) J. St.-Hil. Expos. Fam. Nat. 1(2): 338-340. 1805. Li. MEX (Chis, Gro, Jal, Mich, Nay, Oax, SLP, Sin, Son, Tab, Tam, Ver), MA (Bel, CR), SA. RF, SV, TDF. 0-1,400 m.

\section{Gesneriaceae (1/2)}

Drymonia multiflora (Oerst. ex Hansth.) Wiehler. Phytologia 27(5): 325. 1973. He. MEX (Oax), MA (CR, Nic, Pan). HMF, TEF. 1,000-1,780 m.

D. serrulata (Jacq.) Mart. Nov. Gen. Sp. Pl. 3: 192. 1832. He. MEX (Chis, Col, Jal, Nay, Oax), ANT, MA (Bel, CR, ES, Gua, Hon, Nic, Pan), SA. HMF, TEF, TSEF. 400-1,350 m.

\section{Hernandiaceae (1/1)}

Sparattanthelium amazonum Mart. Flora 24(2, Beibl. 2): 42. 1841. Li, Sc. MEX (Chis, Oax, Tab, Ver, Yuc), MA (CR, Gua, Hon, Nic, Pan), SA. TEF. 0-545 m.

\section{Hydrangeaceae (2/5)}

Hydrangea albostellata Samain, Najarro \& E. Martínez. Phytotaxa 162(4): 183, f. 1-4. 2014. Li. MEX (Chis), MA (CR, ES, Gua, Hon, Nic). HMF, PQF. 1,400-2,520 m.

H. nebulicola Nevling \& Gómez Pompa. J. Arnold. Arbor. 49: 231. 1968. Li. MEX (Qro, Ver). HMF, TEF. 950-1,600 m.

H. steyermarkii Standl. Publ. Field Mus. Nat. Hist. Bot. Ser. 22(4): 233. 1940. He. MEX (Chis), MA (CR, Gua, Hon, Nic, Pan). HMF, TEF. 900-1,700 m. 
Appendix 2. Continuation.

Philadelphus coulteri S. Watson. Proc. Amer. Acad. 22: 472. 1887. Sc. MEX (Gto, Hgo, Qro, SLP, Tam). CF, HMF, QF, SV. 1,000$2,300 \mathrm{~m}$.

P. mexicanus Schltdl. Linnaea 13: 418-419. 1839. Sc. USA, MEX (Chis, DF, Gro, Hgo, Mex, Mich, Mor, Oax, Pue, Qro, Ver), MA (ES, Gua, Hon). CF, HMF, PQF, QF. 1,700-2,800 m.

\section{Lamiaceae (3/7)}

Aegiphila deppeana Steud. Nomencl. Bot. (ed. 2) 1: 29. 1840. Sc. MEX (Chis, Oax, Tab, Ver), MA (CR, Gua, Nic, Pan), SA. RF, SAV, SV, TDF, TEF, TSEF. 0-1,000 m.

A. elata Sw. Prodr. 31. 1788. Sc. MEX (Chis, Oax, Tab, Ver), ANT, MA (Bel, CR, Gua, Hon, Nic, Pan), SA. RF, SV, TEF. 0-500 m.

A. falcata Donn. Sm. Bot. Gaz. 18: 7 (1893). Sc. MEX (Chis), MA (CR, Gua, Nic, Pan). RF, SV, TEF, TSEF. 168 m.

A. panamensis Moldenke. Trop. Woods 25: 14-16. 1931. Sc. MEX (Chis), MA (CR, ES, Gua, Hon, Nic, Pan), SA. SV, TDF, TEF. NA.

Salvia disjuncta Fernald. Proc. Amer. Acad. Arts 35(25): 533. 1900. Sc. MEX (Chis, Mor, Oax), MA (Gua, Nic). HMF, PQF. 1,720$3,000 \mathrm{~m}$.

S. gesneriflora Lindl. \& Paxton. Flow. Gard. 2: 49, t. 47. 1851. Sc. MEX (DF, Gto, Jal, Mex, Mich, Mor, SLP). CF, HMF, PQF, QF. 2,000-3,300 m.

Volkameria ligustrina Jacq. Collectanea 118-119, t. 5, f. 1. 1796[1797]. Sc. MEX (Ver), MA (Bel, Nic, Pan). MG, SV, TDF, TEF, TSEF. 0-600 m.

\section{Loganiaceae (1/4)}

Strychnos brachistantha Standl. Publ. Field Mus. Nat. Hist. Bot. Ser. 12: 412-413. 1936. Li, Sc. MEX (Cam, Chis, Jal, Pue, QR, Tab, Ver), MA (Bel, CR, Gua, Hon, Nic, Pan). HMF, SV, TEF, TSEF. 25-600 m.

S. panamensis Seem. Bot. Voy. Herald 166. 1854. Li. MEX (Cam, Chis, Gro, Jal, Nay, Oax, QR, Sin, Tab, Ver), MA (Bel, CR, ES, Gua, Hon, Nic, Pan), SA. SV, TEF, TSEF. 50-500 m.

S. panurensis Sprague \& Sandwith. Bull. Misc. Inform. Kew 1927(3): 132-133. 1927. Li, Sc. MEX (Ver), MA (Bel, CR, Gua, Pan), SA. HMF, TEF. 750-900 m.

S. peckii B.L. Rob. Proc. Amer. Acad. Arts 49(8): 504. 1913. Li, Sc. MEX (Chis), MA (Bel, CR, Gua, Hon, Nic, Pan), SA. HMF, TEF. $200 \mathrm{~m}$.

\section{Malpighiaceae (14/57)}

Adelphia hiraea (Gaertn.) W.R. Anderson. Novon 16(2): 171. 2006. Li. MEX (Chis, Tab), ANT, MA (Bel, CR, Gua), SA. TEF. 30$500 \mathrm{~m}$.

Banisteriopsis elegans (Triana \& Planch.) Sandwith. J. Arnold Arbor. 24(2): 222. 1943. Li. MEX (Chis), MA (CR, Gua, Hon, Nic, Pan), SA. TDF. 1,000-1,500 m.

B. muricata (Cav.) Cuatrec. Webbia 8: 503. 1958. Li, Sc. MEX (Chis, Oax), MA (Bel, CR, ES, Gua, Hon, Nic, Pan), SA. CF, PQF, TDF, TEF, TSEF. 350-1,100 m.

Bronwenia acapulcensis (Rose) W.R. Anderson \& C. Davis. Contr. Univ. Michigan Herb. 25: 141. 2007. Li, Sc. MEX (Chis, Gro, Oax), MA (CR, ES, Gua, Hon, Nic, Pan), SA. RF, TDF. NA.

B. cornifolia (Kunth) W.R. Anderson \& C. Davis. Contr. Univ. Michigan Herb. 25: 142-143. 2007. Li, Sc. MEX (Chis, Mex, Oax, QR, Tab, Ver), MA (Bel, CR, ES, Gua, Hon, Nic), SA. SAV, TDF, TEF, TSEF. 80-1,100 m.

Callaeum chiapense (Lundell) D.M. Johnson. Syst. Bot. 11(2): 346. 1986. Li. MEX (Chis). 2,100-2,700 m.

C. clavipetalum D.M. Johnson. Syst. Bot. 11(2): 347, f. 1i, 4. 1986. Li, Sc. MEX (Oax). NA.

C. coactum D.M. Johnson. Syst. Bot. 11(2): 342-343, f. 2e, 3. 1986. Li, Sc. MEX (Gro, Mex, Mich, Pue). QF, TDF, TF. 500-1,750 m.

C. johnsonii W.R. Anderson. Acta Bot. Mex. 74: 180-182, f. 1. 2006. Li, Sc. MEX (Pue). TDF. NA.

C. macropterum (Moc. \& Sessé ex DC.) D.M. Johnson. Syst. Bot. 11(2): 340. 1986. Li, Sc. USA, MEX (Ags, BCS, Chih, Col, Gro, Jal, Mich, Nay, NL, Oax, Sin, Son). SV, TDF, XS. 50-1,950 m.

C. malpighioides (Turcz.) D.M. Johnson. Syst. Bot. 11(2): 344-345. 1986. Li. MEX (Chis, Col, Gro, Oax, Ver), MA (Bel, Gua). HMF, QF, TDF, TEF, TSEF, XS. 0-1,400 m.

C. septentrionale (A. Juss.) D.M. Johnson. Syst. Bot. 11(2): 343. 1986. Li, Sc. MEX (Hgo, NL, Oax, Qro, SLP, Tam). PQF, TDF, TEF, TF. 85-2,000 m.

Carolus sinemariensis (Aublet) W.R. Anderson. Novon 16(2): 188. 2006. Li, Sc. MEX (Chis, Jal, Nay, QR), ANT, MA (CR, Nic), SA. TDF, TEF, XS. $830 \mathrm{~m}$.

Christianella mesoamericana (W.R. Anderson) W.R. Anderson. Novon 16(2): 191. 2006. Li. MEX (Chis), MA (CR, Gua, Pan). NA.

Cottsia californica (Benth.) W.R. Anderson \& C. Davis. Contr. Univ. Michigan Herb. 25: 161. 2007. Li. MEX (BCS, Son). TDF, XS. 0-400 m.

C. gracilis (A. Gray) W.R. Anderson \& C. Davis. Smithsonian Contr. Knowl. 3(5): 37. 1852. Li. USA, MEX (BC, Chih, Dgo, NL, Son). XS. 200-1,300 m. 
Appendix 2. Continuation.

Gaudichaudia albida Schltdl. \& Cham. Linnaea 5: 217. 1830. Li. MEX (Cam, Chis, Gro, Jal, Mich, Mor, Nay, Oax, Ver, Yuc), MA (ES, Gua, Hon, Nic), SA. QF, RF, TDF, XS. 180-1,700 m.

G. cycloptera (DC.) W.R. Anderson. Contr. Univ. Michigan Herb. 16: 69. 1987. Li. MEX (Col, Jal, Mich, Oax). HMF, QF. 360$1,400 \mathrm{~m}$.

G. diandra Chodat. Veg. Parag. 203. 1917. Li. MEX (Gro, Oax). TDF. NA.

G. mcvaughii W.R. Anderson. Contr. Univ. Michigan Herb. 16: 72-75, f. 7A-G. 1987. Li. MEX (Col, Gro, Jal, Oax, Ver). TDF. 0$800 \mathrm{~m}$.

Heteropterys brachiata (L.) DC. Prodr. 1: 591. 1824. Sc. MEX (Cam, Chis, Gro, Hgo, Jal, Mich, Mor, Oax, QR, Tab, Yuc), MA (Bel, ES, Gua, Hon, Nic, Pan), SA. HMF, SV, TDF, TEF, TSEF. 3-1,300 m.

H. cotinifolia A. Juss. Ann. Sci. Nat. Bot. sér. 2, 13: 274. 1840. Li. MEX (Chis, Gro, Jal, Mich, Nay, Oax, Sin). RF, TDF. 25-1,000 m.

H. laurifolia (L.) A. Juss. Ann. Sci. Nat. Bot. sér. 2, 13: 276. 1840. Li, Sc. MEX (Chis, Col, Gro, Jal, Mex, Mich, Mor, Oax, QR, Tab, Ver), ANT, MA (CR, ES, Hon, Nic, Pan), SA. RF, TDF, TEF, TSEF. 660-1,050 m.

H. lindeniana A. Juss. Arch. Mus. Hist. Nat. 3: 457. 1843. Li, Sc. MEX (QR, Tab, Yuc), MA (Bel, Gua, Nic). SAV, SV, TDF, TSEF. 0-140 m.

H. macrostachya A. Juss. Ann. Sci. Nat. Bot. sér. 2, 13: 275. 1840. Li, Sc. MEX (Chis, Oax), ANT, MA (Bel, CR, Nic, Pan), SA. TEF, TSEF. 160-300 m.

H. palmeri Rose. Contr. U.S. Natl. Herb. 1(9): 311, f. 2. 1895. Li. MEX (Col, Gro, Jal, Oax, Sin, Son). SAV, TDF, TF. 0-500 m.

H. panamensis Cuatrec. \& Croat. Ann. Missouri Bot. Gard. 67(4): 887, f. 6. 1980[1981]. Li, Sc. MEX (Chis), MA (CR, Nic, Pan). TEF, TSEF. NA.

Hiraea barclayana Benth. Bot. Voy. Sulphur 75. 1844. Li, Sc. MEX (Gro, Oax), MA (Nic). RF, TSEF. NA.

H. fagifolia (DC.) A. Juss. Ann. Sci. Nat. Bot. sér. 2, 13: 258. 1840. Li, Sc. MEX (Chis, Oax, Tab, Ver), ANT, MA (Bel, CR, Gua, Hon, Nic, Pan), SA. SV, TEF, TSEF. 10-890 m.

H. obovata Huber. Bol. Mus. Paraense Hist. Nat. Ethnogr. 3(4): 424-425. 1902. Li. MEX (Oax, QR, Tab, Ver, Yuc), MA (Bel, ES, Gua, Pan). TEF, TSEF. 3-200 m.

H. quapara (Aubl.) Sprague. J. Bot. 62(733): 22. 1924. Li. MEX (Tab, Ver), MA (Bel, Gua, Pan), SA. NA.

H. reclinata Jacq. Enum. Syst. PI. 21. 1760. Li, Sc. MEX (Cam, Chis, Jal, Oax), ANT, MA (Bel, CR, ES, Nic, Pan), SA. CF, HMF, RF, TDF, TEF, TSEF. 500-1,000 m.

H. smilacina Standl. Contr. Arnold Arbor. 5: 87, t. 13. 1933. Li. MEX (Oax, Ver), MA (Bel, CR, Hon, Pan), SA. SV, TEF. 100-520 m.

Mascagnia polycarpa Brandegee. Univ. Calif. Publ. Bot. 10(8): 409. 1924. MEX (Cam, Chis, Oax, Ver), MA (Bel, Gua). TEF, TSEF. 160-200 m.

M. sepium (A. Juss.) Griseb. FI. Bras. 12(1): 96. 1858. Li. MEX (Oax). RF. 120-1,000 m.

M. tomentosa C.E. Anderson. Brittonia 53(3): 411-413, f. 2. 2001. Li. MEX (Chis, Oax, Ver), MA (ES, Gua, Hon, Nic). TDF. 0$19,00 \mathrm{~m}$.

M. vacciniifolia Nied. Arbeiten Bot. Inst. Königl. Lyceums Hosianum Braunsberg 3: 11. 1908. Li. MEX (Chis, Mor, Oax, Ver), MA (Bel, CR, Gua, Hon). SV, TEF. 50-1,250 m.

Psychopterys dipholiphylla (Small) W.R Anderson \& S. Corso. Contr. Univ. Michigan Herb. 25: 118. 2007. Li, Sc. MEX (Gro, Jal, Mex, Mor, Oax, Pue). QF, TDF. 570-2,000 m.

P. mcvaughii W.R. Anderson \& S. Corso. Contr. Univ. Michigan Herb. 25: 121-122. 2007. Li. MEX (Jal). HMF. 1,250 m.

P. multiflora (Nied.) W.R Anderson \& S. Corso. Contr. Univ. Michigan Herb. 25: 122. 2007. Li, Sc. MEX (Chis, Oax), MA (Gua). TDF. 550-1,200 m.

P. ornata W.R. Anderson \& S. Corso. Contr. Univ. Michigan Herb. 25: 125-127, fig. 6. 2007. Li. MEX (Chis). HMF. 1,300 m.

P. rivularis (C.V. Morton \& Standl.) W.R. Anderson \& S. Corso. Contr. Univ. Michigan Herb. 25: 132. 2007. Li, Sc. MEX (Chis, QR, Ver), MA (Bel, Gua). TEF. 0-660 m.

Stigmaphyllon bannisterioides (L.) C.E. Anderson. Taxon 41(2): 328. 1992. Li, Sc. MEX (Tab, Ver), ANT, MA (Bel, CR, Gua, Hon, Nic, Pan), SA. TEF. 5-20 m.

S. dichotomum (L.) Griseb. Linnaea 13: 207-208. 1839. Li. MEX (Chis, Oax, SLP, Tab, Ver), MA (Bel, CR, ES, Gua, Pan), SA. SV, TDF, TSEF. 160-700 m.

S. ellipticum (Kunth) A. Juss. Ann. Sci. Nat. Bot. sér. 2, 13: 290. 1840. Li. MEX (Cam, Chis, Oax, QR, Tab, Tam, Ver, Yuc), ANT, MA (Bel, CR, ES, Gua, Hon, Nic, Pan), SA. PQF, SV, TDF, TEF, TF, TSEF. 10-50 m.

S. lindenianum A. Juss. Arch. Mus. Hist. Nat. 3: 362-363. 1843. Li. MEX (Cam, Chis, Oax, QR, Tab, Ver, Yuc), MA (Bel, CR, Gua, Hon, Nic, Pan), SA. MG, RF, SV, TDF, TEF, TSEF. $10 \mathrm{~m}$.

S. pseudopuberum Nied. Malpigh. Amer. 2: 28. 1912. Li. MEX (Chis, Oax, Tab), MA (Bel, Gua). HMF, PQF. 960-3,200 m.

S. retusum Griseb. Vidensk. Meddel. Dansk Naturhist. Foren. Kjøbenhavn 1853(1-2): 45. 1854. Li. MEX (Chis, Hgo, Oax, Pue, Qro, SLP, Ver), MA (Bel, CR, ES, Gua, Hon, Nic). RF, SV, TEF, XS. 10-760 m.

S. selerianum Nied. Stigmatoph. 1900(2): 7. 1900. Li. MEX (Chis, Oax). TDF, TEF. 550-1,500 m. 
Appendix 2. Continuation.

Tetrapterys arcana C.V. Morton. Publ. Carnegie Inst. Wash. 461: 132. 1936. Li, Sc. MEX (Cam, Chis, Oax), MA (Bel, ES, Gua, Hon, Nic). TDF, TSEF. 90-190 m.

T. discolor (G. Mey.) DC. Prodr. 1: 587. 1824. Li, Sc. MEX (Chis, Ver), ANT, MA (Bel, CR, Gua, Hon, Nic, Pan), SA. RF, TDF, TEF. 100-200 m.

T. goudotiana Triana \& Planch. Ann. Sci. Nat., Bot., sér. 4, 18: 333. 1862. Li. MEX (Chis), MA (Bel, CR, Pan), SA. NA.

T. heterophylla (Griseb.) W.R. Anderson. Contr. Univ. Michigan Herb. 20: 35. 1995. Li. MEX (Chis, Oax, Ver), MA (ES, Gua). RF, TDF, TSEF. 150-1,000 m.

T. mexicana Hook. \& Arn. Bot. Beechey Voy. 281. 1841 [1838]. Li. MEX (Col, Gro, Jal, Mex, Mich, Mor, Nay, NL, Oax, Pue, Sin). TDF, TSEF. 930-1,630 m.

T. schiedeana Schltdl. \& Cham. Linnaea 5(2): 218. 1830. Li, Sc. MEX (Cam, Chis, Hgo, Oax, QR, Yuc), MA (Bel, CR, Gua, Hon, Nic, Pan). HMF, TDF, TEF, TSEF, XS. 0-1,600 m.

T. seleriana Nied. Bot. Jahrb. Syst. 36(3): 18. 1905. Li. MEX (Cam, Oax, Tab, Yuc), MA (Bel, Gua). SAV, SV, TDF, TEF, TSEF. 10$900 \mathrm{~m}$.

T. tinifolia Triana \& Planch. Ann. Sci. Nat. Bot. sér. 4, 18: 336. 1862. Li, Sc. MEX (Chis, Ver), MA (CR, Nic, Pan), SA. RF, SV, TEF. 60-630 m.

\section{Malvaceae (2/4)}

Byttneria aculeata (Jacq.) Jacq. Select. Stirp. Amer. Hist. 76-77. 1763. Li, Sc. MEX (Cam, Chis, Chih, Col, Gro, Hgo, Jal, Mex, Mich, Mor, Nay, Oax, Qro, QR, SLP, Sin, Son, Tab, Tam, Ver, Yuc), MA (Bel, CR, ES, Gua, Hon, Nic, Pan), SA. HMF, RF, SV, TDF, TEF, TSEF. 0-1,400 m.

B. catalpifolia Jacq. subsp. catalpifolia. Pl. Rar. Hort. Schoenbr. 1: 21-22, t. 46. 1797. Li. MEX (Chis, Gro, Jal, Mich, Nay, Oax, Ver, Yuc), ANT, MA (Bel, CR, Gua, Nic, Pan), SA. RF, TDF, TEF, TSEF. 0-1,100 m.

B. fluvialis Fryxell \& Guadarrama. Brittonia 53(1): 59-61, f. 1. 2001. Li. MEX (Tab). NA.

Hibiscus uncinellus DC. Prodr. 1: 449. 1824. Li, Sc. MEX (Chis, Gro, Jal, Nay, Oax, Pue, Sin, Tab, Ver), MA (Gua). HMF, PQF, SV, TDF, TEF. 100-1,700 m.

\section{Marcgraviaceae (3/8)}

Marcgravia brownei (Triana \& Planch.) Krug \& Urb. Symb. Antill. 1: 367. 1899. He. MEX (Chis), ANT, MA (Bel, CR, Gua, Hon, Nic, Pan), SA. HMF, QF, SV. 800-1,700 m.

M. mexicana Gilg. Bot. Jahrb. Syst. 25(Beibl. 60): 26. 1898. He. MEX (Chis, Oax, Ver), MA (CR, Hon, Nic, Pan). HMF, RF, TEF. 50-950 m.

M. nepenthoides Seem. J. Bot. 8: 245. 1870. He. MEX (Chis, Oax, Ver), MA (Bel, CR, Gua, Nic, Pan), SA. TEF. 0-350 m.

M. pittieri Gilg. Bot. Jahrb. Syst. 42: 124. 1908. He. MEX (Oax), MA (CR, Gua, Nic, Pan). TEF. 200 m.

M. stonei Utley. Brenesia 9: 52-53, f. 1. 1976. Li. MEX (Chis, Oax, Ver). HMF. 1,300-1,800 m.

Ruyschia enervia Lundell. Phytologia 1(7): 245-246. 1937. He, Li. MEX (Chis, Ver), MA (Bel, CR, Gua, Hon). HMF, TEF. 650$800 \mathrm{~m}$.

Souroubea exauriculata Delpino. Atti Soc. Ital. Sci. Nat. 12: 180, 200. 1869. Sc, He. MEX (Chis, Oax, Tab, Ver), MA (Bel, Gua, Hon, Nic). HMF, TEF, TSEF. 400-1,000 m.

S. loczyi (V.A. Richt.) de Roon subsp. loczyi. Acta Bot. Neerl. 15(3): 587. 1967. Sc, He. MEX (Cam, Chis, Oax, Tab, Ver), MA (Bel, CR, Gua, Hon, Pan). SV, TDF, TEF. 0-2,400 m.

\section{Melastomataceae (1/1)}

Adelobotrys adscendens (Sw.) Triana. J. Bot. 5: 210. 1867. He, Li. MEX (Chis, Oax, Ver), MA (Bel, CR, Gua, Hon, Nic, Pan), SA. TEF. 120-475 m.

\section{Menispermaceae $(6 / 13)$}

Abuta chiapasensis Krukoff \& Barneby. Mem. New York Bot. Gard. 20(2): 23-24. 1970. Li. MEX (Chis), MA (Gua). TEF, TSEF. 120-1,450 m.

A. panamensis (Standl.) Krukoff \& Barneby. Mem. New York Bot. Gard. 20(2): 22-23. 1970. Li. MEX (Chis, Oax, Tab, Ver), MA (Bel, CR, Gua, Hon, Nic, Pan), SA. HMF, SV, TEF. 50-950 m.

A. steyermarkii (Standl.) Standl. Publ. Field Mus. Nat. Hist. Bot. Ser. 23(4): 156. 1944. Li, Sc. MEX (Chis, Oax), MA (Bel, CR, Gua, Nic). HMF. 455-1,260 m.

Cissampelos grandifolia Triana \& Planch. Ann. Sci. Nat. Bot. sér. 4, 17: 44. 1862. Li. MEX (Cam, Chis, Gro, Mich, Pue, SLP, Tab, Tam, Ver), ANT, MA (CR, Gua, Hon, Nic, Pan), SA. HMF, TDF, TEF. 150-1,650 m. 
Appendix 2. Continuation.

C. pareira L. Sp. PI. 2: 1031-1032. 1753. Li. USA, MEX (Cam, Chis, Col, Gto, Gro, Hgo, Jal, Mex, Mich, Mor, Nay, Oax, Pue, Qro, QR, SLP, Sin, Son, Tab, Tam, Ver, Yuc), ANT, MA (Bel, CR, ES, Gua, Hon, Nic, Pan), SA, AF, AS. HMF, SV, TDF, TEF, TSEF. 0-1,500 m.

C. tropaeolifolia DC. Syst. Nat. 1: 532-533. 1818[1817]. Li. MEX (Chis, Gro, Hgo, Oax, QR, Tab, Ver), MA (Bel, CR, ES, Gua, Hon, Nic, Pan), SA. HMF, RF, SV, TEF. 70-1,170 m.

Cocculus diversifolius DC. Syst. Nat. 1: 523-524. 1818[1817]. Li. USA, MEX (BCS, Chih, Coa, Col, Gto, Gro, Jal, Mex, Mich, Mor, Nay, NL, Oax, Pue, Qro, SLP, Sin, Son, Tam, Ver). RF, SV, TDF, TEF, XS. 15-2,000 m.

Disciphania calocarpa Standl. Publ. Field Mus. Nat. Hist. Bot. Ser. 4(8): 305. 1929. Li. MEX (Chis, Oax, Tab, Ver), MA (Bel, CR, Gua, Hon, Nic). HMF, SV, TEF, TSEF. 100-1,700 m.

D. cardiophylla Standl. Publ. Field Mus. Nat. Hist. Bot. Ser. 22(1): 20. 1940. Li. MEX (Ver), (Pan). HMF, PQF. 2,000-2,200 m.

D. mexicana Bullock. Bull. Misc. Inform. Kew 1936: 388. 1936. Li. MEX (Jal, Mex, Mich, Nay, Sin). TDF. 0-1,300 m.

D. spadicea Barneby. Mem. New York Bot. Gard. 20(2): 143. 1970. Li. MEX (Jal, Oax), MA (CR, ES, Nic). TDF, TSEF. 540-1,300 m.

Menispermum canadense L. Sp. PI. 1: 340. 1753. Li. USA, MEX (NL, Qro). QF. 1,450-1,725 m.

Odontocarya mexicana Barneby. Anales Inst. Biol. Univ. Nac. México 54(1): 153-155. 1983 [1987]. Li. MEX (Chis, Gro, Ver), MA (CR, Pan), SA. HMF, TDF, TEF. 150-1,100 m.

\section{Nyctaginaceae (3/5)}

Grajalesia fasciculata (Standl.) Miranda. Bol. Soc. Bot. México 29: 34. 1965. Sc. MEX (Oax), MA (Nic). HMF, RF, TDF, XS. 30-580 $\mathrm{m}$.

Pisonia aculeata L. var. aculeata. Sp. PI. 2: 1026. 1753. Li, Sc. USA, MEX (Cam, Chis, Chih, Col, Dgo, Gro, Hgo, Jal, Mex, Mich, Mor, Nay, Oax, Pue, Qro, QR, SLP, Sin, Tab, Tam, Ver, Yuc), ANT, MA (Bel, CR, ES, Gua, Hon, Nic, Pan), SA, AF, AS. RF, SV, TDF, TEF, TSEF. 0-2,500 m.

P. capitata (S. Watson) Standl. Contr. U.S. Natl. Herb. 13(11): 388. 1911. Sc. MEX (Chis, Mich, Nay, Sin, Son). TDF, TSEF. 275$600 \mathrm{~m}$.

P. macranthocarpa (Donn. Sm.) Donn. Sm. Bot. Gaz. 20(7): 293. 1895. Li, Sc. MEX (Chis, Jal), ANT, MA (CR, ES, Gua, Hon), SA. TDF. 140-1,350 m.

Pisoniella arborescens (Lag. \& Rodr.) Standl. Contr. U.S. Natl. Herb. 13(11): 385. 1911. Li, Sc. MEX (DF, Gto, Hgo, Jal, Mex, Mich, Oax, Qro, Ver). SV, TDF, XS. 900-2,400 m.

\section{Passifloraceae $(1 / 27)$}

Passiflora adenopoda DC. Prodr. 3: 330. 1828. Li. MEX (Chis, Hgo, Mex, Oax, Pue, Qro, Tam, Ver), MA (Bel, CR, Gua, Hon, Nic, Pan), SA, AS. HMF, TEF. 800-1,350 m.

P. ambigua Hemsl. Bot. Mag. 128: t. 7822. 1902. Li. MEX (Chis, Tab, Ver), MA (Bel, CR, Gua, Hon, Nic, Pan), SA. HMF, TEF. 60-950 m.

P. bicornis Houst. ex Mill. Gard. Dict. (ed. 8) 13. 1768. Li. MEX (Cam, Chis, Oax, Yuc), MA (CR, ES, Gua, Hon, Nic, Pan), SA. SV, TDF. 0-250 m.

P. biflora Lam. Encycl. 3(1): 36. 1789. Li. MEX (Cam, Chis, Gro, Hgo, Jal, Mich, Nay, Oax, QR, SLP, Sin, Tab, Tam, Ver), ANT, MA (Bel, CR, ES, Gua, Nic, Pan), SA. HMF, MG, TDF, TEF. 0-2,400 m.

P. bryonioides Kunth. Nov. Gen. Sp. (quarto ed.) 2: 140-141. 1817. Li. USA, MEX (Chis, Chih, Dgo, Gto, Hgo, Jal, Mex, Mich, Nay, Oax, Pue, Qro, SLP, Son, Tam, Zac). PQF, QF, SV, TDF, XS. 1,600-2,100 m.

P. capsularis L. Sp. Pl. 2: 957. 1753. Li. MEX (Hgo, Jal, Oax, Pue, QR, Ver), ANT, MA (Bel, CR, ES, Gua, Hon, Nic, Pan), SA. HMF, TDF. 1,400 m.

P. coriacea Juss. Ann. Mus. Natl. Hist. Nat. 6: 109, t. 39, f. 2. 1805. Li. MEX (Cam, Chis, Col, Jal, Mex, Mich, Nay, Oax, QR, SLP, Sin, Tab, Tam, Ver, Yuc), MA (Bel, CR, ES, Gua, Hon, Nic, Pan). HMF, SV, TDF, TEF, TSEF. 0-1,900 m.

P. exsudans Zucc. Abh. Math.-Phys. Cl. Königl. Bayer. Akad. Wiss. 2: 342. 1837. Li. MEX (Ags, Coa, DF, Gto, Gro, Hgo, Jal, Mex, Mich, Mor, NL, Oax, Pue, Qro, SLP, Tam, Tla, Ver). PQF, QF, XS. 1,800-2,700 m.

P. hahnii (E. Fourn.) Mast. Trans. Linn. Soc. London 27: 638. 1871. Li. MEX (Chis, Gro, Oax, Tab, Ver), MA (Bel, CR, Gua, Hon, Pan), SA. HMF, TEF, TSEF. 130-2,300 m.

P. helleri Peyr. Linnaea 30(1): 54-55. 1859. Li. MEX (Chis, Oax, Pue, Tab, Ver), MA (Bel, CR, Gua, Nic). HMF, TEF, TSEF. 25$1,500 \mathrm{~m}$.

P. holosericea L. Sp. PI. 2: 958. 1753. Li. MEX (Chis, Col, Gro, Jal, Mich, Nay, Oax, Sin, Ver), ANT, MA (CR, ES, Gua, Hon, Nic), SA. SV, TDF, TSEF. 0-700 m.

P. jorullensis Kunth. Nov. Gen. Sp. (quarto ed.) 2: 133. 1817. Li. MEX (Chis, Col, Gro, Jal, Mex, Mich, Nay, Oax), MA (ES). HMF, QF. 900-2,000 m.

P. juliana J.M. MacDougal. Novon 2(4): 358, f. 1. 1992. Li. MEX (Col, Jal, Mich). SV, TDF. 20-800 m. 
Appendix 2. Continuation.

P. mexicana Juss. Ann. Mus. Natl. Hist. Nat. 6: 108-109, pl. 38, f. 2. 1805. Li. USA, MEX (Chis, Col, Gro, Jal, Mex, Mich, Nay, Oax, Pue, Sin, Son, Ver). MG, SV, TDF. 0-2,000 m.

P. nelsonii Mast. \& Rose. Contr. U.S. Natl. Herb. 5: 142, t. 17. 1897. Li. MEX (Chis, Ver), MA (CR, Gua, Nic, Pan). HMF. 7001,500 m.

P. obovata Killip. Publ. Carnegie Inst. Wash. 461(13): 308-309, t. 1. 1936. Li. MEX (Oax, QR), MA (Bel, CR, Gua, Hon). 1,140$1,300 \mathrm{~m}$.

P. oerstedii Mast. FI. Bras. 13(1): 562. 1872. Li. MEX (Chis, Ver), MA (Bel, CR, Gua, Hon, Nic, Pan), SA. HMF, TDF. $280-1,370$ m.

P. pavonis Mast. J. Bot. 21: 35. 1883. Li. MEX (Chis, Gro, Jal, Mex, Mich, Mor, Oax), MA (Gua). HMF, PQF, QF, SV. 1,800$2,713 \mathrm{~m}$.

P. porphyretica Mast. Bot. Gaz. 20: 538, t. 36. 1895. Li. MEX (Chis, Col, Gro, Jal, Mich, Nay, Oax, Sin, Son), MA (Gua, Pan). QF, TDF. 100-1,500 m.

P. serratifolia L. Sp. PI. 2: 955. 1753. Li. MEX (Cam, Chis, Oax, Pue, Qro, QR, SLP, Tab, Tam, Ver, Yuc), MA (Bel, CR, Gua, Hon, Nic). QF, TDF, TEF. 5-900 m.

P. sexflora Juss. Ann. Mus. Natl. Hist. Nat. 6: 110-111, pl. 37, f. 1. 1805. Li. MEX (Chis, Oax, Ver), ANT, MA (Bel, CR, Gua, Hon, Nic, Pan), SA. CF, HMF, QF, TEF. 470-2,700 m.

P. sicyoides Schltdl. \& Cham. Linnaea 5: 88. 1830. Li. MEX (Chis, Hgo, Mich, Nay, NL, Oax, Pue, Qro, Ver), MA (Gua), SA. HMF, PQF. 2,000 m.

P. standleyi Killip. J. Wash. Acad. Sci. 14: 110. 1924. Li. MEX (Chis), MA (CR, ES, Gua, Hon, Nic). HMF, PQF, SV. 1,200-1,400 m.

P. suberosa L. Sp. PI. 2: 958. 1753. Li. USA, MEX (Ags, Cam, Chis, Chih, Coa, Col, Dgo, Gto, Gro, Hgo, Jal, Mex, Mich, Mor, Nay, NL, Oax, Pue, Qro, QR, SLP, Sin, Tam, Ver, Yuc), ANT, MA (Bel, CR, ES, Gua, Hon, Nic, Pan), SA. QF, SV, TDF, TF, TSEF, XS. $10-2,330 \mathrm{~m}$.

P. subpeltata Ortega. Nov. PI. Descr. Dec. 6: 78. 1798. Li. MEX (Chis, DF, Gro, Hgo, Jal, Mex, Mich, Mor, Nay, Oax, Pue, Qro, SLP, Ver, Zac,), ANT, MA (Gua, Pan), SA. HMF, PQF, QF, SV, TDF, XS. 1,000-2,300 m.

P. xiikzodz J.M. MacDougal subsp. xiikzodz. Novon 2(4): 361, f. 2-4. 1992. Li. MEX (Cam, QR, Yuc), MA (Bel, Gua). TEF, TSEF. 30-400 m.

P. yucatanensis Killip. Publ. Field Mus. Nat. Hist., Bot. Ser. 8: 26. 1930. Li. MEX (Cam, QR, Yuc). SV, TSEF. 9-150 m.

\section{Phytolaccaceae (2/2)}

Agdestis clematidea Moc. \& Sessé ex DC. Syst. Nat. 1: 543. 1818[1817]. Li. USA, MEX (Chis, Col, Hgo, Jal, Nay, NL, Oax, Pue, Qro, SLP, Sin, Tab, Tam, Ver, Yuc), ANT, MA (CR, Gua, Hon, Nic). QF, RF, SV, TDF, TSEF. 30-1,200 m.

Trichostigma octandrum (L.) H. Walter. Pflanzenr. IV. 83(Heft 39): 109. 1909. Li, Sc. USA, MEX (Chis, Col, Gro, Jal, Mex, Mich, Mor, Oax, Pue, Qro, Sin, Tab, Tam, Ver), ANT, MA (Bel, CR, Gua, Hon, Nic, Pan), SA. HMF, SV, TDF, TEF, TSEF. 50-1,000 m.

\section{Piperaceae (1/2)}

Piper silvivagum C. DC. Prim. Fl. Costaric. 2(3): 242-243. 1899. Li, Sc. MEX (Mor), MA (CR, Nic, Pan), SA. NA.

P. xanthostachyum C. DC. Prim. FI. Costaric. 2(3): 261-262. 1899. He. MEX (Chis, Oax), MA (ES, Gua, Hon, Nic, Pan), SA. NA.

\section{Plantaginaceae (1/1)}

Russelia contrerasii B.L. Turner. Phytologia 81(5): 335-338, f. 2-3. 1996. Li, Sc. MEX (Chis), MA (Gua). HMF, TDF. 400-1,200 m.

\section{Polemoniaceae (1/9)}

Cobaea biaurita Standl. Contr. U.S. Natl. Herb. 17(5): 457. 1914. Li. MEX (Chis, Oax, Ver). CF, HMF, QF. 700-3,500 m.

C. lutea D. Don. Edinburgh Philos. J. 10: 112. 1824. Li. MEX (Chis, Col, Gro, Jal), MA (CR, ES, Gua, Hon), SA. HMF, TDF. 430$1,850 \mathrm{~m}$.

C. minor M. Martens \& Galeotti. Bull. Acad. Roy. Sci. Bruxelles 12(2): 276. 1845. Li. MEX (Ver), MA (CR, Pan). HMF, PQF. 2,000$3,000 \mathrm{~m}$.

C. pachysepala Standl. Contr. U.S. Natl. Herb. 17(5): 456, pl. 29. 1914. Li. MEX (Chis), MA (Gua). HMF. 2,000-3,000 m.

C. paneroi Prather. Brittonia 48(1): 116-117, f. 2. 1996. Li. MEX (Hgo, Mor, Pue). HMF, PQF, SV. 1,200-1,700 m.

C. pringlei (House) Standl. Contr. U.S. Natl. Herb. 17(5): 457. 1914. Li. MEX (Gto, NL, Qro, SLP, Tam). HMF, PQF, QF. 1,800$2,500 \mathrm{~m}$.

C. scandens Cav. Icon. 1: 11, pl. 16-17. 1791[1791]. Li. MEX (Chis, Gro, Hgo, Mex, Mich, Mor, Oax, Pue, Ver), ANT, MA (CR, Gua, Hon, Pan), SA. HMF, PQF, QF, SV, TEF, TSEF. 0-2,500 m.

C. skutchii I.M. Johnst. J. Arnold Arbor. 19(2): 128. 1938. Li. MEX (Chis), MA (Gua). HMF. 1,200-1,800 m.

C. stipularis Benth. PI. Hartw. 45. 1840. Li. MEX (Hgo, Oax, Qro, Ver). HMF, QF. 1,450-2,300 m. 
Appendix 2. Continuation.

Polygalaceae (3/4)

Bredemeyera lucida (Benth.) Klotzsch ex Hassk. Ann. Mus. Bot. Lugduno-Batavi 1(6): 189-190. 1864. Sc. MEX (Cam, Chis, Oax), ANT, MA (Bel, ES, Gua, Hon, Nic, Pan), SA. QF, RF, SAV, TEF. 120-300 m.

Podopterus cordifolius Rose \& Standl. Proc. Biol. Soc. Wash. 33: 66. 1920. Li, Sc. MEX (Col, Jal, Oax). TDF, TSEF. 0-160 m.

Securidaca diversifolia (L.) S.F. Blake. Contr. U.S. Natl. Herb. 23(3): 594. 1923. Li, Sc. MEX (Cam, Chis, Jal, Mich, Oax, QR, Tab), ANT, MA (Bel, Gua, Hon, Nic, Pan), SA. PQF, TEF, TSEF. 0-1,990 m.

S. sylvestris Schltdl. Linnaea 14: 381-382. 1840. Li, Sc. MEX (Chis, Mich, Oax), MA (Bel, CR, Gua, Hon, Nic, Pan). TDF. 50$1,810 \mathrm{~m}$.

\section{Polygonaceae (2/4)}

Antigonon flavescens S. Watson. Proc. Amer. Acad. Arts 22: 446-447. 1887. Li. MEX (Chis, Col, Gro, Jal, Mich, Oax, Qro, SLP, Sin, Ver), MA (Gua). RF, SV, TDF, XS. 300-1,950 m.

A. guatimalense Meisn. Prodr. 14: 184. 1856. Li. MEX (Chis), MA (CR, ES, Gua, Nic, Pan). 250 m.

A. leptopus Hook. \& Arn. Bot. Beechey Voy. 308-309, t. 69. 1841 [1838]. Li. MEX (BC, BCS, Cam, Chis, Chih, Dgo, Gro, Hgo, Jal, Mor, Nay, NL, Oax, Qro, Sin, Son, Tab, Tam, Ver, Yuc), MA (Bel, CR, Gua, Hon, Nic, Pan). PQF, SV, TDF. 0-900 m.

Muehlenbeckia tamnifolia (Kunth) Meisn. PI. Vasc. Gen. 2: 227. 1840[1841]. Li. MEX (Chis, Gro, Mor, Oax, Ver), MA (CR, ES, Gua, Hon, Pan), SA. HMF, PQF. 1,300-3,090 m.

\section{Ranunculaceae (1/11)}

Clematis acapulcensis Hook. \& Arn. Bot. Beechey Voy. 410. 1841[1840]. Li. MEX (Chis, Col, DF, Gro, Hgo, Jal, Mex, Mich, Mor, Nay, Oax, Pue, SLP, Sin, Tam, Ver), MA (CR, ES, Gua, Hon, Nic, Pan), SA. HMF, PQF, QF, TDF, TSEF. 0-1,800 m.

C. caleoides Standl. \& Steyerm. Publ. Field Mus. Nat. Hist. Bot. Ser. 23(2): 52-53. 1944. Li. MEX (Chis), MA (Gua). HMF, PQF. 1,600-3,000 m.

C. coahuilensis D.J. Keil. Bull. Torrey Bot. Club 104: 10. 1977. Li. MEX (Coa, Dgo, NL, SLP). 1,000-1,600 m.

C. dioica L. Syst. Nat. (ed. 10) 2: 1084. 1759. Li, Sc. USA, MEX (Cam, Chis, Col, DF, Gro, Hgo, Jal, Mex, Mich, Mor, Nay, Oax, Pue, QR, Tab, Tam, Ver, Yuc, Zac), ANT, MA (Bel, CR, ES, Gua, Hon, Pan), SA. CF, HMF, PQF, QF, RF, SV, TDF, TEF, TSEF, XS. 0-2,900 m.

C. drummondii Torr. \& A. Gray. Fl. N. Amer. 1(1): 9. 1838. Li. USA, MEX (Ags, BCS, Chih, Coa, Dgo, NL, Oax, Pue, Qro, SLP, Sin, Son, Tam, Zac). PQF, TDF, XS. 990-1,900 m.

C. grahamii Benth. Pl. Hartw. 5. 1839. Li. MEX (Hgo, Mich, NL, Pue, SLP, Tam, Ver). PQF. 1,450-2,800 m.

C. grossa Benth. Pl. Hartw. 33-34. 1840. Li. MEX (Cam, Chis, DF, Hgo, Jal, Mex, Mich, Mor, Nay, NL, Oax, Pue, Qro, SLP, Tam, Ver), MA (CR, ES, Gua, Hon, Nic, Pan). HMF, SV, TDF. 900-3,000 m.

C. ligusticifolia Nutt. FI. N. Amer. 1(1): 9. 1838. Li. USA, MEX (BC, BCS, Chih, Coa). 80-1,215 m.

C. pauciflora Nutt. FI. N. Amer. 1(1): 9. 1838. Li. USA, MEX (BC, BCS). XS. 190-1125 m.

C. polygama Jacq. Enum. Syst. PI. 24. 1760. Li. MEX (Cam, Chis, Hgo, Mor, Oax, Pue, QR, SLP, Tab, Tam, Ver), ANT, MA (Bel, CR, Gua, Hon, Nic, Pan). HMF, SV, TDF, TEF. 0-2,270 m.

C. rhodocarpa Rose. Contr. U.S. Natl. Herb. 10(3): 95. 1906. Li. MEX (Chis, Chih, Col, DF, Gto, Gro, Hgo, Jal, Mex, Mich, Mor, Oax, Pue, Qro, Sin, Tam, Ver, Zac), ANT, MA (Gua, Hon). HMF, PQF, QF, SV, TDF. 1,200-3,000 m.

\section{Rhamnaceae (2/7)}

Gouania conzattii Greenm. Publ. Field Columb. Mus., Bot. Ser. 2(6): 257. 1907. Li. MEX (Mex, Nay, Oax, Pue, Tla), MA (Gua). HMF, QF, TDF. 400-2,200 m.

G. eurycarpa Standl. Publ. Field Mus. Nat. Hist. Bot. Ser. 4(8): 315. 1929. Li, Sc. MEX (Cam, Chis, Oax, QR, Yuc), MA (Bel, CR, Gua, Hon). MG, SV, TDF, TEF, TSEF. $200 \mathrm{~m}$.

G. lupuloides (L.) Urb. Symb. Antill. 4(3): 378. 1910. Li, Sc. USA, MEX (Cam, Chis, Chih, Col, Gro, Hgo, Jal, Mor, Nay, Oax, Qro, QR, SLP, Tab, Tam, Ver, Yuc), ANT, MA (CR, Gua, Hon, Nic, Pan), SA. SV, TDF, TEF, TSEF. 0-1,400 m.

G. polygama (Jacq.) Urb. Symb. Antill. 4(3): 378-379. 1910. Li, Sc. MEX (Cam, Chis, Col, Jal, Nay, Oax, Qro, QR, SLP, Tab, Ver), ANT, MA (Bel, CR, Gua, Hon, Nic, Pan), SA. QF, RF, SV, TDF, TEF, TSEF. 100-1,500 m.

G. rosei Wiggins. Contr. Dudley Herb. 4(2): 20. 1950. Li. MEX (BCS, Chih, Col, Gro, Jal, Nay, Oax, Sin, Son). TDF, TSEF. 40$1,100 \mathrm{~m}$.

G. stipularis DC. Prodr. 2: 39. 1825. Li, Sc. MEX (Chis, Gro, Jal, Oax, Ver, Yuc). RF, SV, TDF, TEF, TSEF. 250-1,185 m.

Sageretia elegans (Kunth) Brongn. Mém. Fam. Rhamnées 53. 1826. Sc. MEX (Cam, Chis, Gro, Jal, Mex, Mich, Oax, Pue, Qro, QR, Sin, Tab, Ver), MA (Bel, CR, Gua, Hon, Nic), SA. PQF, QF, TDF, TEF. 20-1,725 m. 
Appendix 2. Continuation.

\section{Rosaceae (1/4)}

Rubus adenotrichos Schltdl. Linnaea 13: 267-268. 1839. Sc. MEX (Chis, Col, Gro, Hgo, Jal, Mex, Mich, Mor, Oax, Pue, Ver), MA (CR, Gua, Hon, Nic, Pan), SA. CF, HMF, QF, SV. 2,100-2,800 m.

R. fagifolius Schltdl. \& Cham. Linnaea 5: 571. 1830. Sc. MEX (Chis, Oax, Ver), MA (Bel, ES, Gua, Hon). HMF, QF, SV, TEF. 930$2,700 \mathrm{~m}$.

R. philyrophyllus Rydb. N. Amer. FI. 22(5): 451. 1913. Sc. MEX (Hgo, Oax, Pue, Qro, Ver). HMF, QF, SV. 1,350-1,800 m.

R. sapidus Schltdl. Linnaea 13: 269. 1839. Sc. MEX (Chis, Hgo, Mich, Oax, Pue, Qro, Ver), MA (Gua, Hon, Nic). CF, HMF, QF, RF, SV. 1,200-2,050 m.

\section{Rubiaceae (10/21)}

Chiococca alba (L.) Hitchc. Rep. (Annual) Missouri Bot. Gard. 4: 94. 1893. Li, Sc. USA, MEX (Cam, Chis, Col, Gro, Hgo, Jal, Mex, Mich, Mor, Nay, NL, Oax, Qro, QR, SLP, Sin, Tab, Tam, Ver, Yuc), ANT, MA (Bel, CR, Gua, Hon, Nic, Pan), SA. HMF, MG, SV, TDF, TEF, TSEF. 0-1,500 m.

C. belizensis Lundell. Amer. Midl. Naturalist 29(2): 492. 1943. Li, Sc. MEX (Cam, Chis, Oax, QR, Tab, Ver), MA (Bel, CR, Gua, Hon, Nic, Pan), SA. HMF, SV, TDF, TEF, TSEF. 0-1,500 m.

C. coriacea M. Martens \& Galeotti. Bull. Acad. Roy. Sci. Bruxelles 11(1): 231. 1844. Sc. MEX (Cam, QR, Tam, Ver). MG. 0-10 m.

C. phaenostemon Schltdl. Linnaea 9: 594-595. 1835. Sc. MEX (Chis, Gro, Oax, Ver), MA (CR, Gua, Hon, Nic). HMF, TSEF. 9002,000 m.

C. rubriflora Lundell. Wrightia 5(1): 7. 1972. Li, Sc. MEX (Chis), MA (Gua). TEF. 80-160 m.

C. semipilosa Standl. \& Steyerm. Publ. Field Mus. Nat. Hist. Bot. Ser. 22(4): 279. 1940. Li, Sc. MEX (Tam), MA (CR, ES, Gua, Hon, Nic). TDF. NA.

Coutarea hexandra (Jacq.) K. Schum. Fl. Bras. 6(6): 196-197. 1889. Li. MEX (Cam, Chis, Oax, QR, Tab, Ver, Yuc), ANT, MA (Bel, CR, ES, Gua, Hon, Nic, Pan), SA. TDF, TSEF. 3-1,000 m.

Didymaea floribunda Rzed. Bol. Soc. Bot. México 44: 73-75, f. 1983. Li. MEX (DF, Gro, Jal, Mex, Mich, Oax, Ver). HMF, PQF, QF, SV. 1,800-2,750 m.

Guettarda tikalana Lundell. Wrightia 2(2): 63. 1960. Sc. MEX (Chis, Ver), MA (Bel, Gua). TEF, TSEF. 150-600 m.

Hintonia octomera (Hemsl.) Bullock. Icon. Pl. 33(4): 6-7, sub t. 3295. 1935. Sc. MEX (Cam, QR, Yuc), MA (Gua). TDF, TSEF. 2-250 m.

Morinda royoc L. Sp. Pl. 1: 176. 1753. Sc. USA, MEX (Cam, Chis, QR, Tab, Yuc), ANT, MA (Bel, Gua, Hon, Nic, Pan), SA. SV, TDF, TSEF. 2-350 m.

Paederia ciliata (Bartl. ex DC.) Standl. Publ. Field Mus. Nat. Hist. Bot. Ser. 22(5): 386. 1940. Li. MEX (Col, DF, Gro, Mex, Mich, Mor, Oax, Pue). TDF, TF, TSEF. 800-2,010 m.

Randia armata (Sw.) DC. Prodr. 4: 387. 1830. Sc. MEX (Chis, Jal, Oax, QR, Tab), ANT, MA (CR, ES, Gua, Hon, Nic, Pan), SA. TDF, TSEF. 7-1,500 m.

R. cinerea (Fernald) Standl. Contr. U.S. Natl. Herb. 20(6): 201. 1919. Sc. MEX (Chis, Gro, Mich, Oax), MA (Gua, Hon). TDF. 200-1,080 m.

R. guerrerensis Lorence \& Rodr. Acosta. Biótica 11(3): 195-199, f. 1. 1986. Li, Sc. MEX (Gro). RF, TDF, TSEF, XS. 0-1,700 m.

R. longiloba Hemsl. Biol. Cent.-Amer. Bot. 4: 101. 1887. Sc. MEX (Cam, QR, Yuc), MA (Gua). TDF, TSEF. 0-300 m.

R. retroflexa Lorence \& M. Nee. Brittonia 39(3): 371-375, f. 1-2. 1987. Li, Sc. MEX (Chis, Oax, Ver), MA (Bel, CR, Pan). HMF, TEF. 150-850 m.

R. tetracantha (Cav.) DC. Prodr. 4: 387. 1830. Li, Sc. MEX (Chis, Col, Dgo, Gro, Jal, Mich, Nay, Oax, Pue, Sin, Ver). CF, HMF, RF, TDF, TSEF. 0-1,700 m.

R. vazquezii Lorence \& Dwyer. Bol. Soc. Bot. México 47: 46-48, f. 3. 1987. Li. MEX (Chis, Oax, Ver), MA (CR, Gua, Hon, Pan). HMF, TEF, TSEF. 30-900 m.

Rogiera edwardsii (Standl.) Borhidi. Acta Bot. Hung. 43(1-2): 44. 2001. Sc. MEX (Chis, Oax), (Hon). CF, HMF, PQF. 900 m.

Sabicea panamensis Wernham. Monogr. Sabicea 30. 1914. Li, Sc. MEX (), MA (Bel, CR, Gua, Hon, Nic, Pan), SA. NA.

S. villosa Willd. ex Roem. \& Schult. Syst. Veg. 5: 265. 1819. Li. MEX (Chis, Mex, Tab), MA (Bel, CR, Gua, Hon, Nic, Pan), SA. TEF. NA.

\section{Rutaceae (1/1)}

Zanthoxylum foliolosum Donn. Sm. Bot. Gaz. 18(2): 1. 1893. Li, Sc. MEX (Chis, Hgo), MA (ES, Gua, Hon, Nic). HMF, PQF, TEF, XS. 560-2,800 m.

\section{Sapindaceae (6/48)}

Balsas guerrerensis Cruz Durán \& K. Vega. Novon 21(2):199. 2011. Li. MEX (Gro). QF, TDF. 1,420-1,555 m.

Cardiospermum grandiflorum Sw. Prodr. 64. 1788. Li. USA, MEX (Cam, Chis, Hgo, Mex, Mich, Oax, Tab, Ver, Zac), ANT, MA (Bel, CR, Gua, Hon, Pan), SA, AF. SV, TDF, XS. 0-1,720 m. 
Appendix 2. Continuation.

C. halicacabum L. Sp. Pl. 1: 366-367. 1753. Li. MEX (BC, BCS, Cam, Chis, Chih, Coa, Col, DF, Dgo, Gto, Gro, Hgo, Jal, Mex, Mich, Mor, Nay, NL, Oax, Pue, Qro, QR, SLP, Sin, Son, Tab, Tam, Tla, Ver, Yuc, Zac), ANT, MA (Gua, Hon), SA, AF, AS. PQF, QF, RF, SAV, SV, TDF, TF, TSEF, XS. 680-2,250 m.

Paullinia clavigera Schltdl. Linnaea 10: 239-240. 1836. Li. MEX (Cam, Chis, Jal, Nay, Oax, QR, Ver), MA (Bel, ES, Gua, Hon, Nic), SA. TEF, TSEF. 0-1,700 m.

P. costaricensis Radlk. Abh. Math.-Phys. Cl. Königl. Bayer. Akad. Wiss. 16(1): 157. 1886. Li. MEX (Chis, Col, Oax, QR, Tab, Ver), MA (Bel, CR, Gua, Hon, Nic, Pan). HMF, SV, TDF, TEF. 2-1,000 m.

P. costata Schltdl. \& Cham. Linnaea 5(2): 216-217. 1830. Li. MEX (Chis, Oax, Ver), MA (Bel, CR, Gua, Nic, Pan), SA. SV, TEF. 60-1,000 m.

P. cururu L. Sp. PI. 1: 365-366. 1753. Li. MEX (Cam, Chis, Gro, Jal, Mich, Oax, QR), ANT, MA (Bel, CR, Gua, Hon, Nic, Pan), SA. CF, HMF, RF, SV, TDF, TEF, TSEF. 5-20 m.

P. fuscescens Kunth var. fuscescens. Nov. Gen. Sp. 5: 120. 1821. Li. MEX (Cam, Chis, Col, Jal, Mor, Oax, QR, Ver), ANT, MA (Bel, ES, Gua, Hon, Nic, Pan), SA. HMF, SV, TDF, TEF. 750-1,050 m.

P. fuscescens Kunth var. glabrata Croat. Ann. Missouri Bot. Gard. 63(3): 480. 1976[1977]. Li. MEX (Cam, Jal, Ver), MA (Nic, Pan), SA. TDF, TEF, TSEF. 4-1,600 m.

P. pinnata L. Sp. Pl. 1: 366. 1753. Li. MEX (Cam, Chis, Oax, QR, Tab, Yuc), ANT, MA (Bel, ES, Gua, Hon, Pan), SA, AF. SV, TDF, TEF, TSEF. $20 \mathrm{~m}$.

P. sessiliflora Radlk. Contr. U.S. Natl. Herb. 1: 317. 1895. Li. MEX (Col, Jal, Mich, Oax, QR), MA (Nic, Pan). SV, TEF, TSEF. 700$1,350 \mathrm{~m}$.

P. tomentosa Jacq. Enum. Syst. Pl. 37. 1760. Li, Sc. MEX (Cam, Chis, Col, Gro, Hgo, Jal, Qro, QR, Sin, Tam, Ver, Yuc), MA (Bel, Gua, Hon). HMF, QF, SV, TDF, TSEF. 250-1,400 m.

P. turbacensis Kunth. Nov. Gen. Sp. (quarto ed.) 5: 114. 1821. Li. MEX (Chis), MA (CR, Gua, Hon, Nic, Pan), SA. TEF. 800$1,000 \mathrm{~m}$.

P. venosa Radlk. Abh. Math.-Phys. Cl. Königl. Bayer. Akad. Wiss. 19: 114, 230. 1896. Li. MEX (Ver), ANT, SA. SV, TEF. $160-220$ m.

Serjania acuta Triana \& Planch. Ann. Sci. Nat. Bot. sér. 4, 18: 349. 1862. Li. MEX (Chis), MA (CR, Nic, Pan), SA. SV, TSEF. $1,290 \mathrm{~m}$.

S. adiantoides Radlk. Publ. Field Columb. Mus. Bot. Ser. 1(4): 403. 1898. Li. MEX (Cam, Chis, QR, Yuc), MA (Bel, Gua). SV, TDF, TEF, TSEF. 6-1,500 m.

S. atrolineata C. Wright. Anales Acad. Ci. Med. Habana 5: 292-293. 1868. Li. MEX (Chis, Gro, Nay, Oax, QR, Sin, Tab, Ver, Yuc), ANT, MA (Bel, CR, Gua, Hon, Nic, Pan), SA. HMF, SV, TDF, TEF, TSEF. 35-1,450 m.

S. brachycarpa A. Gray ex Radlk. Monogr. Serjania 259. 1875. Li. USA, MEX (Jal, Mich, NL, SLP, Tam, Ver). SV, TDF. 20-400 m.

S. brachylopha Radlk. Contr. U.S. Natl. Herb. 1(9): 367-368. 1895. Li. MEX (Jal). TDF. 100 m.

S. cambessedeana Schltdl. \& Cham. Linnaea 5: 214. 1830. Li. MEX (Hgo, Oax, Qro, SLP, Ver), MA (Gua). QF, TDF, TSEF. 700$800 \mathrm{~m}$.

S. caracasana (Jacq.) Willd. Sp. Pl. 2(1): 465. 1799. Li. MEX (Chis, Oax, QR), ANT, MA (Bel, CR, ES, Gua, Hon, Nic), SA. HMF, RF, TSEF. 20-1,700 m.

S. cardiospermoides Schltdl. \& Cham. var. cardiospermoides. Linnaea 6: 418. 1831. Li. MEX (Chis, Gro, Oax, Qro, SLP, Ver), MA (CR, ES, Gua, Hon, Nic). HMF, QF, TDF, TSEF. 250-1,300 m.

S. cardiospermoides Schltdl. \& Cham. var. subjubata (Radlk.) Acev.-Rodr. Mem. New York Bot. Gard. 67: 71. 1993. Li. MEX (Chis), MA (Gua). PQF, TDF, TEF, TSEF. 0-2,700 m.

S. flaviflora Radk. Bull. Herb. Boissier, sér. 2, 3: 211. 1903. Li. MEX (Col, Jal, Mich, Oax, Ver). PQF, TDF, TSEF. 1,068-2,140 m.

S. goniocarpa Radlk. Monogr. Serjania 309-310. 1875. Li. MEX (Cam, Chis, Col, Gro, Hgo, Jal, Mich, Oax, QR, Tab, Ver, Yuc), MA (Bel, Gua, Hon). PQF, SV, TDF, TEF, XS. 5-2,030 m.

S. grosii Schltdl. Linnaea 18: 42 [printed on page as 58]. 1844. Li. MEX (Cam, Chis, Col, Gro, Jal, Mich, Nay, Oax, Tab, Ver, Yuc), MA (Bel, Gua, Hon, Nic). SV, TDF, TSEF. 18-1,800 m.

S. lobulata Standl. \& Steyerm. Publ. Field Mus. Nat. Hist. Bot. Ser. 23(1): 14. 1943. Li. MEX (Chis, Mich, Nay, Oax, Pue), MA (CR, Gua, Hon, Nic). SV, TDF, TSEF. 185-2,100 m.

S. Iundellii Croat. Phytologia 33(3): 170. 1976. Li. MEX (Cam, Chis, Gro, Jal), MA (Bel, Gua). TDF, TEF, XS. 200-1,300 m.

S. macrocarpa Standl. \& Steyerm. Publ. Field Mus. Nat. Hist. Bot. Ser. 23(1): 15. 1943. Li. MEX (Chis, Gro, Mich, Oax, Tab), MA (Bel, CR, Gua, Hon, Nic). SV, TEF. 150-700 m.

S. mexicana (L.) Willd. Sp. PI. 2: 465. 1799. Li. MEX (Chis, Jal, Mex, Mich, QR, Tab, Ver, Zac), ANT, MA (Bel, CR, ES, Gua, Hon, Nic, Pan), SA. HMF, SV, TEF. 940-1,040 m.

S. oaxacana Standl. Contr. U.S. Natl. Herb. 23(3): 695. 1923. Li. MEX (Oax). NA.

S. palmeri S. Watson. Proc. Amer. Acad. Arts 24: 45. 1889. Li. MEX (Son). TDF, XS. 85-405 m.

S. paniculata Kunth. Nov. Gen. Sp. (quarto ed.) 5: 86. 1821. Li. MEX (Cam, Chis), MA (CR, Nic, Pan), SA. TDF, TEF. $300-900$ m.

S. paucidentata DC. Prodr. 1: 603. 1824. Li. MEX (Chis, Oax, Ver), ANT, MA (Bel, CR, Nic, Pan), SA. TEF. $130-825$ m. 
Appendix 2. Continuation.

S. plicata Radlk. Contr. U.S. Natl. Herb. 23(3): 695. 1923. Li. MEX (Ver). TEF. 150 m.

S. psilophylla Radlk. Bot. Gaz. 16(6): 191-192. 1891. Li. MEX (Chis, Jal, Oax), MA (Gua). CF, HMF, TEF. 1,700-1,950 m.

S. pterarthra Standl. Publ. Carnegie Inst. Wash. 461(4): 70. 1935. Li. MEX (Cam, Chis, QR, Tab), MA (Bel, Gua). TDF, TEF. 5$114 \mathrm{~m}$.

S. punctata Radlk. ex Donn. Sm. Bot. Gaz. 20(7): 281. 1895. Li. MEX (Chis, Nay, Ver), MA (Gua, Hon). QF, TDF, TEF, TSEF. 60$2,600 \mathrm{~m}$.

S. racemosa Schumach. Skr. Naturhist.-Selsk. 3(2): 127, t. 12, f. 3. 1794. Li, Sc. MEX (Cam, Chis, Gto, Gro, Jal, Mex, Mich, Mor,

NL, Oax, Pue, Qro, QR, SLP, Sin, Tam, Ver, Yuc, Zac), MA (CR, Gua, Hon, Nic). HMF, QF, SV, TDF, TEF, XS. 550-2,400 m.

S. rachiptera Radlk. Bot. Gaz. 16(6): 192. 1891. Li. MEX (Cam, Chis), MA (ES, Gua, Hon, Nic). HMF, TSEF. 160-1,450 m.

S. rekoi Standl. Contr. U.S. Natl. Herb. 23(3): 696. 1923. Li. MEX (Oax, Ver). TEF. 60 m.

S. rhombea Radlk. Monogr. Serjania 324-326. 1875. Li. MEX (Chis, Tab), MA (CR, Hon, Nic, Pan), SA. TEF. 120-350 m.

S. rhytidococca Acev.-Rodr. Brittonia 49: 500. 1997. Li. MEX (Gro). TDF. 900 m.

S. schiedeana Schltdl. Linnaea 18: 44. 1844. Li. MEX (Ags, Gro, Jal, Mex, Mich, Mor, Oax, Zac), MA (Nic). SV, TDF. 10-1,500 m.

S. subtriplinervis Radlk. Monogr. Serjania 273. 1875. Li. MEX (Oax). NA.

S. trifoliolata Radlk. Contr. U.S. Natl. Herb. 1(9): 317. 1895. Li. MEX (Gro, Mex, Sin), MA (ES, Hon, Pan). 265-1,500 m.

S. triquetra Radlk. Monogr. Serjania 305-309. 1875. Li. MEX (Cam, Chis, Gro, Jal, Mex, Mich, Mor, Nay, Oax, Pue, QR, Sin, Tab, Ver, Yuc, Zac), MA (CR, ES, Gua, Hon, Nic), SA. QF, SV, TDF, XS. 1,800-1,830 m.

S. yucatanensis Standl. Publ. Field Mus. Nat. Hist. Bot. Ser. 8(1): 21. 1930. Li. MEX (Cam, Chis, QR, Yuc), MA (Bel, Gua). SV, TDF, TSEF. 5-20 m.

Thinouia tomocarpa Standl. Publ. Field Mus. Nat. Hist., Bot. Ser. 12: 411. 1936. Li. MEX (Cam, Chis, QR, Ver), MA (Bel, Gua, Hon, Pan), SA. SV, TEF, TSEF. 120-280 m.

Urvillea ulmacea Kunth. Nov. Gen. Sp. (quarto ed.) 5: 106, pl. 440. 1821. Li, Sc. USA, MEX (Cam, Chis, Col, Gto, Gro, Hgo, Jal, Mex, Mich, Mor, Nay, NL, Oax, Pue, Qro, QR, SLP, Sin, Tab, Tam, Ver, Yuc), ANT, MA (Bel, CR, ES, Gua, Hon, Nic, Pan), SA. QF, RF, SV, TDF, TEF, TF, TSEF, XS. 10-1,820 m.

\section{Schisandraceae (1/1)}

Schisandra glabra (Brickell) Rehder. J. Arnold Arbor. 25(1): 131. 1944. Li. USA, MEX (BCS, Hgo). HMF. 1600 m.

\section{Schlegeliaceae (1/2)}

Schlegelia nicaraguensis Standl. Trop. Woods 16: 44. 1928. He. MEX (Ver), MA (CR, Nic, Pan). HMF, TEF. 0-800 m.

S. parviflora (Oerst.) Monach. Phytologia 3(3): 103. 1949. He. MEX (Oax, Ver), MA (Bel, CR, Gua, Hon, Nic, Pan), SA. SV, TEF. $130-250 \mathrm{~m}$.

\section{Siparunaceae $(1 / 1)$}

Siparuna gesnerioides (Kunth) A. DC. Prodr. 16(2.2): 646. 1868. Li, Sc. MEX (Chis, Gro, Oax, Ver), MA (CR, Pan), SA. CF, HMF, TEF. 250-1,600 m.

\section{Smilacaceae (1/11)}

Smilax aristolochiifolia Mill. Gard. Dict. (ed. 8) 7. 1768. Li, Sc. MEX (Cam, Chis, Hgo, Mich, Oax, Pue, Qro, SLP, Tab, Tam, Ver, Yuc), MA (Bel, CR, Gua). HMF, TSEF. 300-1,400 m.

S. bona-nox L. Sp. PI. 2: 1030. 1753. Li, Sc. USA, MEX (Chis, Coa, Hgo, NL, Oax, Qro, SLP, Tam, Ver), ANT. HMF, PQF, QF, RF. 1,100-2,000 m.

S. domingensis Willd. Sp. PI. 4(2): 783. 1806. Li, Sc. USA, MEX (Cam, Chis, Col, Gro, Hgo, Jal, Mich, Nay, NL, Oax, Pue, Qro, Tab, Tam, Ver), ANT, MA (Bel, CR, ES, Gua, Hon, Nic, Pan). HMF, PQF, QF, RF, TDF, TEF, TSEF. 70-2,000 m.

S. glauca Walter. Fl. Carol. 245. 1788. Li, Sc. USA, MEX (Chis, Hgo, Oax, Pue, Qro, SLP, Tam, Ver). CF, HMF, QF. 1,200-1,700 m.

S. mollis Humb. \& Bonpl. ex Willd. Sp. Pl. 4: 785. 1806. Li, Sc. MEX (Cam, Chis, Gro, Hgo, Jal, Mex, Mich, Mor, Oax, Pue, Qro, QR, SLP, Sin, Tab, Tam, Ver, Yuc), MA (Bel, CR, Gua, Hon, Nic, Pan), SA. CF, HMF, MG, PQF, QF, SV, TDF, TEF, TSEF. 5-2,500 m.

S. moranensis M. Martens \& Galeotti. Bull. Acad. Roy. Sci. Bruxelles 9(2): 389. 1842. Li. MEX (Chis, Coa, Col, DF, Dgo, Gto, Gro, Hgo, Jal, Mex, Mich, Mor, Nay, NL, Oax, Pue, Qro, SLP, Sin, Son, Tam, Tla, Ver), MA (ES, Gua, Hon, Nic). CF, HMF, PQF, QF, SV, TSEF. 1,000-3,100 m.

S. ornata Lem. Ill. Hort. 12: t. 439. 1865. Li. MEX (Chis, Col, Oax, Tab), MA (Bel, Gua, Hon, Nic). CF, HMF, TEF. NA.

S. spinosa Mill. Gard. Dict. (ed. 8) 8. 1768. Li, Sc. MEX (Cam, Chis, Col, Gro, Jal, Mich, Nay, Oax, Pue, Qro, QR, Sin, Tab, Tam, Ver, Yuc), MA (Bel, CR, ES, Gua, Hon, Nic, Pan). RF, SV, TDF, TEF, TSEF. 250-1,900 m.

S. subpubescens A. DC. Monogr. Phan. 1: 69. 1878. Li. MEX (Cam, Chis, Gro, Mex, Mich, Oax, Pue, Tab, Ver), MA (CR, ES, Gua, Hon, Nic, Pan). HMF, PQF, QF, TEF. 750-2600 m. 
Appendix 2. Continuation.

S. tomentosa Kunth. Nov. Gen. Sp. (quarto ed.) 1: 272. 1815[1816]. Li. MEX (Hgo, Tab), SA. HMF. NA.

S. velutina Killip \& C.V. Morton. Publ. Carnegie Inst. Wash. 461(12): 283. 1936. Li. MEX (Chis, Oax, Tab), MA (Bel, CR, Gua, Hon, Nic). RF, TEF, TSEF. 360-1,770 m.

\section{Solanaceae $(6 / 35)$}

Cestrum scandens Vahl. Eclog. Amer. 1: 24-25. 1797. Li. MEX (Chis, Oax, Ver), MA (Bel, CR, ES, Gua, Hon, Nic, Pan), SA. SV, TDF, TEF. 30-260 m.

Juanulloa mexicana (Schltdl.) Miers. Ann. Mag. Nat. Hist. ser. 2 4: 188-189. 1849. Sc, He. MEX (Chis, Oax, Tab, Ver), MA (CR, ES, Gua, Hon, Pan), SA. TDF, TEF, TSEF. 0-755 m.

Lycianthes anomala Bitter. Lycianthes 514-515. 1919. Sc, He. MEX (Oax, Ver), MA (Nic). TEF, TSEF. 800-1,100 m.

L. armentalis J.L. Gentry. Phytologia 26(4): 269-270. 1973. Sc. MEX (Cam, Chis, Oax, QR, Tab, Ver, Yuc), MA (Bel, Gua, Hon). SV, TDF, TSEF. 102-764 m.

L. chiapensis (Brandegee) Standl. Publ. Field Mus. Nat. Hist. Bot. Ser. 11(5): 173. 1936. Li, Sc. MEX (Chis, Ver), MA (Gua, Nic). HMF, TDF. 700-2,730 m.

L. hypoleuca Standl. Trop. Woods 9: 12. 1927. Sc. MEX (Cam, Chis, QR), MA (Bel, Gua, Hon). SV, TDF, TEF, TSEF. 172-1,160 m.

L. lenta (Cav.) Bitter. Lycianthes 364-366. 1919. Li, Sc. MEX (Cam, Chis, Oax, Tab, Ver), ANT, MA (Bel, CR, Gua, Hon, Nic), SA. SV, TDF, TEF, TSEF. 0-300 m.

L. purpusii (Brandegee) Bitter. Lycianthes 382-385, 299-300, f. 2, 4. 1919. Li. MEX (Chis, Oax, Tab, Ver), MA (Bel, Gua, Hon). SV, TEF, TSEF. 100-1,200 m.

L. sideroxyloides (Schltdl.) Bitter. Lycianthes 403-405. 1919. Li. MEX (Cam, Chis, Oax, QR, Ver), MA (Bel, Hon). QF, TDF. 750$1,300 \mathrm{~m}$.

Solandra grandiflora Sw. Kongl. Vetensk. Acad. Nya Handl. 8: 303-306, t. 11. 1787. Ar, He, Li. MEX (Chis, Gro, Jal, Oax, Tab, Ver), ANT, MA (CR, ES, Gua, Hon, Nic, Pan), SA. HMF, PQF. 450-2,700 m.

S. guerrerensis Martinez. Anales Inst. Biol. Univ. Nac. Autón. México, Bot. 37: 101. 1967. Ar, He, Li. MEX (DF, Gro, Hgo, Mex, Mich, Mor, Oax, Ver). CF, HMF, QF. 500-2400 m.

S. guttata D. Don. Edwards's Bot. Reg. 18: pl. 1551. 1832. He, Li. MEX (DF, Dgo, Gro, Hgo, Jal, Mex, Mich, Mor, NL, Oax, Qro, Sin, Tam, Ver, Zac). HMF, PQF, QF, TDF, TSEF. 1,250-2,800 m.

S. maxima (Sessé \& Moc.) P.S. Green. Bot. Mag. 176(3): t. 506. 1967. Ar, He, Li. MEX (Chis, Col, Gro, Hgo, Jal, Mex, Mich, Mor, Oax, Pue, Qro, Sin, Tab, Ver), MA (Bel, CR, ES, Gua, Hon, Nic, Pan), SA. HMF, PQF, TDF, TEF, TSEF. $100-2,300$ m.

S. nizandensis Matuda. Anales Inst. Biol. Univ. Nac. México 43(1): 60, f. 8. 1972 [1974]. Sc, He. MEX (Chis, Jal, Mich, Oax), MA (Gua, Hon). PQF, RF, TDF, XS. 0-3,000 m.

Solanum aculeolatum M. Martens \& Galeotti. Bull. Acad. Roy. Sci. Bruxelles 12(1): 143. 1845. Li. MEX (Chis, Gro, Oax), MA (Gua). TDF. 0-1,100 m.

S. adhaerens Willd. ex Roem. \& Schult. Syst. Veg. 4: 669. 1819. Li. MEX (Cam, Chis, Ver), MA (Bel, Gua, Nic, Pan), SA. SV, TEF, TSEF. 0-400 m.

S. appendiculatum Dunal. Solan. Syn. 5. 1816. Li. MEX (Chis, DF, Gro, Hgo, Jal, Mex, Mich, Oax, Qro, Tam, Ver), MA (Gua, Hon, Nic, Pan). HMF, PQF, QF, TEF. 1,200-2,800 m.

S. aturense Dunal. Solan. Syn. 32. 1816. Li, Sc. MEX (Chis, Oax, Ver), MA (Bel, Gua, Nic, Pan), SA. SV, TEF. 120-1,200 m.

S. cobanense J.L. Gentry. Phytologia 26(4): 276. 1973. Li. MEX (Chis), MA (Gua, Hon, Nic). HMF. 900-2,550 m.

S. dulcamaroides Dunal. Encycl. Suppl. 3: 751. 1814. Li. MEX (Chis, Gto, Jal, Mich, Oax, Ver), MA (Gua, Nic), SA. HMF, QF,

TDF, TEF, TSEF. 300-2,100 m.

S. guamuchilense Cast.-Campos \& Rzed. Acta Bot. Mex. 27: 33-37. 1994. Li. MEX (Nay). TSEF. 300 m.

S. hamatile Brandegee. Univ. Calif. Publ. Bot. 6(8): 192. 1915. Li. MEX (Gro, Oax, Pue). TDF. 10-1400 m.

S. ionidium Bitter. Repert. Spec. Nov. Regni Veg. 11: 484-485. 1913. Li. MEX (DF, Gro, Oax, Pue, Ver). CF, PQF. 2,300-2,900 m.

S. jamaicense Mill. Gard. Dict. (ed. 8) 17. 1768. Li, Sc. USA, MEX (Chis, Gro, Oax, Tab, Ver), ANT, MA (Bel, CR, Gua, Hon, Nic, Pan), SA. QF, SAV, SV, TEF, TSEF. 90-920 m.

S. lanceifolium Jacq. Icon. PI. Rar. 2(1): pl. 329. 1788. Li, Sc. MEX (Cam, Chis, Col, Oax, SLP, Tab, Tam, Ver), ANT, MA (Bel, CR, Gua, Hon, Nic, Pan), SA. TDF, TEF, TSEF. 0-50 m.

S. refractum Hook. \& Arn. Bot. Beechey Voy. 304. 1841 [1838]. Li. MEX (Col, Gro, Jal, Mich, Oax). SV, TDF, TSEF. $200-1,290$ m.

S. seaforthianum Andrews. Bot. Repos. 8: pl. 504. 1808. Li. MEX (Chis, Oax, Tab, Ver, Yuc), ANT, MA (Bel, CR, Gua, Hon, Nic, Pan), SA, AF. HMF, TDF, TEF. 0-1300 m.

S. skutchii Correll. Contr. Texas Res. Found. Bot. Stud. 1(1): 4-6, t. 1. 1950. Li. MEX (Hgo, Mich, Oax, Qro, Ver), MA (Gua, Hon, Nic). HMF, PQF, TEF. 900-1,300 m.

S. sousae S. Knapp. PLoS ONE 5(5): e10502, [6, 7-8; fig. 5]. 2010. Li. MEX (Oax, Pue). HMF. 1,600-1,900 m.

S. suaveolens Kunth \& C.D. Bouché. Sp. Nov. Hort. Berol. 1848 14. 1848 [1849]. Li. MEX (Chis, Oax, SLP, Ver), MA (Bel, CR, Gua, Hon), SA. PQF, SV, TEF. 650-900 m. 
Appendix 2. Continuation.

S. subvelutinum Rydb. Bull. Torrey Bot. Club 51: 175. 1924. Li. MEX (Dgo, Sin). CF. 800-2,440 m.

S. tacanense Lundell. Amer. Midl. Naturalist 29(2): 490-491. 1943. Li. MEX (Chis), MA (Gua). 2,100 m.

S. tampicense Dunal. Prodr. 13(1): 284. 1852. Li, Sc. USA, MEX (Gro, Oax, Tab, Tam, Ver, Yuc), ANT, MA (Bel, CR, ES, Gua). MG, SV. 0-50 m.

S. wendlandii Hook. f. Bot. Mag. 113: t. 6914. 1887. Li. MEX (Chis, Oax, Tab, Ver), MA (CR, Gua, Hon, Nic, Pan), SA. SV, TDF, TEF. 100-850 m.

Witheringia stellata (Greenm.) Hunz. Kurtziana 5: 129. 1969. Sc. MEX (Hgo, Oax, Ver). HMF, PQF. 1,525-2,300 m.

\section{Stegnospermataceae $(1 / 3)$}

Stegnosperma cubense A. Rich. Hist. Fís. Cuba, Bot. 10: 309. 1845. Sc. MEX (BC, Chis, Col, Gro, Jal, Mex, Mich, Nay, Oax, Sin, Ver), ANT, MA (CR, ES, Gua, Nic). TDF. 0-400 m.

S. halimifolium Benth. Bot. Voy. Sulphur 17, t.12. 1844. Sc. MEX (BC, BCS, Col, Jal, Mex, Mich, Nay, Oax, Son). TDF, XS. 0$480 \mathrm{~m}$.

S. watsonii D.J. Rogers. Ann. Missouri Bot. Gard. 36:475. 1949. Sc. MEX (Sin, Son). TDF. 0-320 m.

\section{Trigoniaceae (1/2)}

Trigonia eriosperma (Lam.) Fromm \& E. Santos subsp. membranacea (A.C. Sm.) Lleras. Fl. Neotrop. 19: 47. 1978. Li, Sc. MEX (Cam, Chis, Oax), MA (Bel, CR, Gua, Nic, Pan), SA. TEF, TSEF. 70-400 m.

T. rugosa Benth. Bot. Voy. Sulphur 74. 1844. Li, Sc. MEX (Chis), MA (CR, ES, Gua, Hon, Nic, Pan), SA. TEF, TSEF, XS. $100-500$ m.

\section{Urticaceae (1/3)}

Urera killipiana Standl. \& Steyerm. Fieldiana, Bot. 24(3): 427. 1952. Li, Sc. MEX (Chis, Hgo, Oax, Qro, Tab, Ver), MA (ES, Gua). HMF, RF, SV. 900-3,000 m.

U. lianoides A.K. Monro \& Al. Rodr. Ann. Missouri Bot. Gard. 96(2): 278-281, f. 2A-F. 2009. Li, Sc. MEX (Chis, Ver), MA (CR, Gua, Hon, Nic, Pan), SA. HMF, SV, TEF. 0-2,500 m.

U. simplex Wedd. Prodr. 16(1): 90. 1869. Li, Sc. MEX (Chis, Tab, Ver), ANT, MA (CR, Nic, Pan), SA. HMF, SV, TEF. 0-2,500 m.

\section{Verbenaceae (1/1)}

Petrea volubilis L. Sp. PI. 2: 626. 1753. Li, Sc. MEX (Cam, Chis, Coa, Gro, Hgo, Mich, Mor, Oax, Pue, Qro, QR, SLP, Tab, Tam, Ver, Yuc), ANT, MA (Bel, CR, ES, Gua, Hon, Nic, Pan), SA. HMF, RF, SV, TDF, TEF, TSEF. 0-1,800 m.

\section{Violaceae (1/1)}

Corynostylis arborea (L.) S.F. Blake. Contr. U.S. Natl. Herb. 23(3): 837. 1923. Li. MEX (Chis, Gro, Oax, QR, Tab, Ver, Yuc), MA (Bel, CR, Gua, Hon, Nic, Pan), SA. MG, RF, TEF. 0-20 m.

\section{Vitaceae (5/26)}

Ampelocissus acapulcensis (Kunth) Planch. Monogr. Phan. 5: 403-404. 1887. Li. MEX (Chis, Col, Gro, Jal, Mex, Mich, Mor, Nay, Oax, Pue, Sin), MA (ES, Gua). QF, SV, TDF, TSEF. 30-2,000 m.

A. erdvendbergiana Planch. Vigne Amér. Vitic. Eur. 9(3): 94-95. 1885. 1887. Li. MEX (Chis, Oax, Pue, Qro, SLP, Tab, Tam, Ver, Yuc), MA (ES, Gua). QF, TDF, TSEF. 14-1,600 m.

A. mesoamericana Lombardi. Novon 15(4): 566. 2005. Li. MEX (Chis, Ver), MA (ES, Gua, Hon). TDF, TEF, TSEF. 60-1,350 m.

Ampelopsis denudata Planch. Monogr. Phan. 5: 619. 1887. Li, Sc. MEX (Chis, Gro, Jal, Mex, Mich, Nay, Oax, Sin, Son, Ver), MA (Gua). SV, TDF, TEF. 60-1,400 m.

Cissus alata Jacq. Select. Stirp. Amer. Hist. 23, pl. 182, f. 10. 1763. Li. MEX (Cam, Chis, Gro, Jal, Mor, Oax, Qro, QR, Tab, Ver, Yuc), ANT, MA (Bel, CR, Gua, Hon, Nic, Pan), SA. HMF, PQF, RF, SV, TDF, TEF, XS. 5-50 m.

C. biformifolia Standl. Publ. Field Mus. Nat. Hist. Bot. Ser. 4(8): 225-226. 1929. Li. MEX (Chis, Col, Mor, Oax, Tab, Ver), MA (Bel, CR, ES, Gua, Hon, Nic, Pan), SA. SV, TEF, TSEF. 10-1,550 m.

C. cacuminis Standl. Publ. Field Mus. Nat. Hist. Bot. Ser. 17(4): 375. 1938. Li. MEX (Chis, Gro, QR, Tab), MA (Bel, CR, Gua, Hon, Nic). CF, HMF, TEF. 1,000-1,900 m.

C. cucurbitina Standl. Contr. U.S. Natl. Herb. 23(3): 732. 1923. Li. MEX (Gro, Jal, Mex, Mor, Nay, Oax, Sin), MA (ES, Nic). QF, SV, TDF, TSEF. 100-1,550 m.

C. erosa Rich. Actes Soc. Hist. Nat. Paris 1: 106. 1792. Li, Sc. MEX (Chis, Oax, QR, Tab, Ver), ANT, MA (Bel, CR, ES, Gua, Hon, Nic, Pan), SA. HMF, QF, TEF, TSEF. 37-2,356 m.

C. gossypiifolia Standl. Publ. Field Mus. Nat. Hist. Bot. Ser. 8(1): 23. 1930. Li. MEX (Cam, Chis, Gro, Oax, QR, Tab, Ver, Yuc), MA (Bel, CR, Gua, Nic), SA. SV, TDF, TEF, TSEF. 0-327 m.

C. mexicana DC. Prodr. 1: 631. 1824. Li. MEX (BCS, Nay, Sin, Son). TDF. 110-825 m. 
Appendix 2. Continuation.

C. microcarpa Vahl. Eclog. Amer. 1: 16. 1796[1797]. Li. MEX (Ags, Cam, Chis, Col, Gro, Jal, Mex, Mich, Mor, Nay, Oax, Pue, Qro, QR, SLP, Sin, Tab, Tam, Ver, Yuc), ANT, MA (Bel, CR, ES, Gua, Hon, Nic, Pan), SA. QF, SV, TDF, TEF, TSEF. 0-1,150 m.

C. ulmifolia (Baker) Planch. Monogr. Phan. 5(2): 552. 1887. Li. MEX (Tab, Ver), MA (Be, CR, Gua, Hon, Pan), SA. 0-1,200 m.

C. tiliacea Kunth. Nov. Gen. Sp. (quarto ed.) 5: 222-223. 1821 [1822]. Li, Sc. MEX (DF, Gto, Gro, Hgo, Jal, Mex, Mich, Mor, Nay, Oax, Pue, Qro, Son, Ver), MA (Nic). QF, SV, TDF, TF, XS. 0-2,400 m.

C. trianae Planch. Monogr. Phan. 5: 555-556. 1887. Li. MEX (Chis, Gro, Oax), MA (CR, ES, Gua, Hon, Nic, Pan), SA. HMF, TDF, TF. 1,700-2,400 m.

C. trifoliata (L.) L. Syst. Nat. (ed. 10) 2: 897. 1759. Li. USA, MEX (BCS, Cam, Chih, Coa, Col, Dgo, Gto, Gro, Jal, Mich, NL, Oax, Pue, Qro, QR, SLP, Sin, Son, Tam, Ver, Yuc), ANT, MA (Nic), SA. TDF, TSEF, XS. 550-1,000 m.

C. verticillata (L.) Nicolson \& C.E. Jarvis subsp. verticillata. Taxon 33(4): 727. 1984. Li, Sc. USA, MEX (Cam, Chis, Col, DF, Dgo, Gro, Hgo, Jal, Mex, Mich, Mor, Nay, NL, Oax, Pue, Qro, QR, SLP, Sin, Son, Tab, Tam, Ver, Yuc), ANT, MA (Bel, CR, ES, Gua, Hon, Nic, Pan), SA. HMF, RF, SV, TDF, TEF, TSEF, XS. 0-2,500 m.

Parthenocissus quinquefolia (L.) Planch. Monogr. Phan. 5(2): 448. 1887. Li. USA, MEX (Chis, Chih, Coa, Col, Dgo, Gto, Gro, Hgo, Jal, Mich, NL, Oax, Pue, Qro, SLP, Sin, Son, Tam, Ver, Yuc), MA (Gua). CF, HMF, QF, TDF, XS. 800-2,400 m.

Vitis arizonica Engelm. Amer. Naturalist 2(6): 321. 1868. Li. USA, MEX (Chih, Coa, Dgo, Son). PQF, QF. 920-2,600 m.

V. berlandieri Planch. Compt. Rend. Hebd. Séances Acad. Sci. 91: 425-428. 1880. Li. USA, MEX (Dgo, Jal, Mich, Tam). HMF, RF. 1,500-2,020 m.

V. bourgaeana Planch. Monogr. Phan. 5: 368. 1887. Li, Sc. MEX (Chis, Gro, Hgo, Jal, Mich, Mor, Oax, Qro, QR, Tab, Ver), MA (Bel, Gua). HMF, TSEF. 0-2,600 m.

V. cinerea (Engelm.) Engelm. ex Millardet. Mém. Soc. Sci. Phys. Nat. Bordeaux 2: 319. 1880. Li. USA, MEX (Coa, Gro, Mex, Mich, Nay, NL, Oax, Tam, Ver). HMF, PQF, RF. 240-2,200 m.

V. girdiana Munson. Proc. Soc. Promot. Agric. Sci. 8: 59. 1887. Li. USA, MEX (BCS). XS. 190 m.

V. peninsularis M.E. Jones. Contr. W. Bot. 18: 57. 1933. Li. MEX (BCS). CF, TDF. 600-1,950 m.

V. popenoei J.L. Fennell. J. Wash. Acad. Sci. 30(1): 17-19, f. 2. 1940. Li. MEX (Chis, Hgo, Oax, Pue, Qro, Tab, Ver), MA (Bel, Gua). HMF, QF, SV. 800-1650 m.

V. tiliifolia Humb. \& Bonpl. ex Roem. \& Schult. Syst. Veg. 5: 320. 1819. Li. MEX (BCS, Cam, Chis, Col, Dgo, Gto, Gro, Hgo, Jal, Mex, Mich, Mor, Nay, Oax, Pue, Qro, QR, SLP, Sin, Tab, Ver, Yuc), ANT, MA (Bel, CR, ES, Gua, Hon, Nic, Pan), SA. HMF, QF, RF, SV, TDF, TEF, TSEF. 250-2,300 m. 